\title{
Resummation of double-differential cross sections and fully-unintegrated parton distribution functions
}

\author{
Massimiliano Procura, ${ }^{a}$ Wouter J. Waalewijn ${ }^{b, c}$ and Lisa Zeune ${ }^{b}$ \\ ${ }^{a}$ Albert Einstein Center for Fundamental Physics, Institute for Theoretical Physics, \\ University of Bern, \\ CH-3012 Bern, Switzerland \\ ${ }^{b}$ ITFA, University of Amsterdam, \\ Science Park 904, 1018 XE, Amsterdam, The Netherlands \\ ${ }^{c}$ Theory Group, Nikhef, \\ Science Park 105, 1098 XG, Amsterdam, The Netherlands \\ E-mail: mprocura@itp.unibe.ch, wouterw@nikhef.nl, l.k.zeune@uva.nl
}

ABSTRACT: LHC measurements involve cuts on several observables, but resummed calculations are mostly restricted to single variables. We show how the resummation of a class of double-differential measurements can be achieved through an extension of Soft-Collinear Effective Theory (SCET). A prototypical application is $p p \rightarrow Z+0$ jets, where the jet veto is imposed through the beam thrust event shape $\mathcal{T}$, and the transverse momentum $p_{T}$ of the $Z$ boson is measured. A standard SCET analysis suffices for $p_{T} \sim m_{Z}^{1 / 2} \mathcal{T}^{1 / 2}$ and $p_{T} \sim \mathcal{T}$, but additional collinear-soft modes are needed in the intermediate regime. We show how to match the factorization theorems that describe these three different regions of phase space, and discuss the corresponding relations between fully-unintegrated parton distribution functions, soft functions and the newly defined collinear-soft functions. The missing ingredients needed at NNLL/NLO accuracy are calculated, providing a check of our formalism. We also revisit the calculation of the measurement of two angularities on a single jet in JHEP 1409 (2014) 046, finding a correction to their conjecture for the NLL cross section at $\mathcal{O}\left(\alpha_{s}^{2}\right)$.

KEYwORDS: Resummation, Effective field theories, QCD

ARXIV EPRINT: 1410.6483 


\section{Contents}

1 Introduction 1

2 Factorization 5

2.1 Effective theory for the region between $\mathrm{SCET}_{\mathrm{I}}$ and $\mathrm{SCET}_{\mathrm{II}}$ boundaries 5

2.2 Factorization formulae 8

3 Ingredients at NNLL $\quad 11$

$\begin{array}{lll}3.1 \text { Hard function } & 11\end{array}$

3.2 Beam functions 12

3.3 Soft functions 13

$\begin{array}{ll}3.4 \text { Collinear-soft function } & 15\end{array}$

$\begin{array}{ll}3.5 & \text { Renormalization and anomalous dimensions } \\ \end{array}$

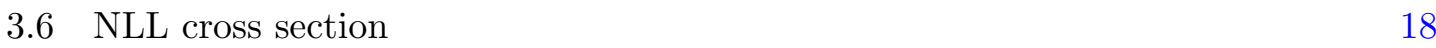

4 Matching the effective theories 19

$5 \quad$ NLO cross section $\quad 21$

5.1 Ingredients 22

5.2 Cancellation of IR divergences 24

5.3 Result 25

5.4 Comparison to resummed predictions 26

6 Measuring two angularities on one jet 29

$\begin{array}{lll}7 & \text { Conclusions } & 32\end{array}$

A Plus distributions $\quad 33$

B Renormalization group evolution $\quad 33$

\section{Introduction}

Experimental LHC analyses typically involve several kinematic cuts. Many of them are fairly harmless from a theoretical point of view. However, when these restrictions on initialand/or final-state radiation lead to widely separated energy scales, large logarithms can be induced in the corresponding cross section, requiring resummation. One example is given by the jet veto used to suppress backgrounds in Higgs analyses, where the resummation of jet-veto logarithms [1-6] greatly reduces the dominant source of theoretical uncertainty. A closely related process is Drell-Yan (or vector boson) production in the case the lepton 
pair has a small $p_{T}$ compared to their invariant mass $Q$ [7-17]. Another example is the jet mass $m_{J}$ spectrum of a jet with transverse momentum $p_{T}^{J}$, which requires resummation around the peak of the distribution where $m_{J} \ll p_{T}^{J}[18-21]$.

In this paper we focus on double differential measurements, where both observables lead to large logarithms. Using effective field theory methods, we derive new resummed expressions for a class of double differential cross sections. Our results smoothly connect to the phase space boundaries, which require different effective field theories. This formalism has applications to jet cross sections and jet substructure studies, and we will consider an example of both in this paper.

As the field of jet substructure has matured [22-24], multivariate analyses have become common. Furthermore, some of the measurements with the best discrimination power are ratios of infrared and collinear safe observables, such as ratios of $N$-subjettiness [25-27], energy correlation functions [28-30] or planar flow [31,32]. These quantities are themselves not infrared and collinear safe, and their calculation involves marginalizing over the resummed two-dimensional distribution [33]. The pioneering study in ref. [34], investigating the measurement of two angularities on one jet, inspired the present paper.

Our formalism can also be applied to $p p \rightarrow H+1$ jet production, where in addition to the jet veto the transverse momentum of the jet becomes small. This important contribution to the cross section is not yet fully understood [6]. In this paper, to better illustrate the features of our framework, we will mainly focus on a simpler (but related) problem in $Z+0$ jet production, carrying out the simultaneous resummation of the jet veto and the transverse momentum of the $Z$ boson.

Resummation is often achieved using the parton shower formalism. The great advantage of parton shower Monte Carlo event generators, such as Pythia [35] and Herwig [36], is that they produce a fully exclusive final state, giving the user full flexibility. On the other hand, this approach is limited to leading logarithmic (LL) accuracy, and it is difficult to estimate the corresponding theory uncertainty. It is also not clear to what extent correlations between resummed observables are correctly predicted by Monte Carlo models, see e.g. ref. [37]. By contrast, we predict these correlations and our resummed predictions have a theory uncertainty attached to it, whose reliability can be verified by comparing different orders in resummed perturbation theory. Note that there has been significant progress by matching higher-order matrix elements with parton showers (see e.g. refs. [38-45]) and (partially) including higher-order resummation [42].

We will illustrate the features of our framework in the specific case of $p p \rightarrow Z+0$ jets, where the transverse momentum $p_{T}$ of the $Z$ boson is measured and a global jet veto is imposed using the beam thrust event shape $[1,46]$

$$
\mathcal{T}=\sum_{i} p_{i T} e^{-\left|\eta_{i}\right|}=\sum_{i} \min \left\{p_{i}^{+}, p_{i}^{-}\right\}
$$

The sum on $i$ runs over all particles in the final state, except for the leptonic decay products of the $Z$. Here, $p_{i T}$ is the magnitude of the transverse momentum and $\eta_{i}$ the pseudorapidity of particle $i$ in the center-of-mass frame of the hadronic collision. Light-cone coordinates 


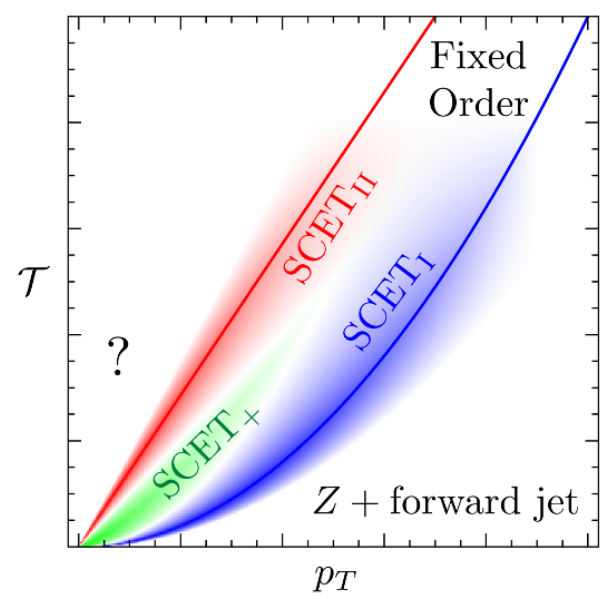

Figure 1. The different regions for the double measurement of $p_{T}$ and beam thrust $\mathcal{T}$ in $Z$-boson production from $p p$ collisions.

are defined as

$$
p_{i}^{\mu}=p_{i}^{+} \frac{n^{\mu}}{2}+p_{i}^{-} \frac{n^{\mu}}{2}+p_{i \perp}^{\mu}, \quad p_{i}^{-}=\bar{n} \cdot p_{i}, \quad p_{i}^{+}=n \cdot p_{i},
$$

where $n^{\mu}=(1,0,0,1)$ and $\bar{n}^{\mu}=(1,0,0,-1)$ are along the beam axis. Beam thrust imposes a global veto on all radiation in an event, which is impractical in the LHC environment. This can be remedied by only including the contributions from jet regions in eq. (1.1) [47]. We will nevertheless consider the global veto to keep our discussion as simple as possible. At the end of section 2.2 we will comment on a special class of non-global measurements whose logarithms can easily be resummed within our approach.

We will perform resummations using Soft-Collinear Effective Theory (SCET) [48-51]. Which version of SCET is the appropriate one, namely what the relevant degrees of freedom are, depends on the region of phase space probed by the measurement, as shown in figure 1 and discussed below. We find that in the intermediate region, between the $\mathrm{SCET}_{\mathrm{I}}$ and $\mathrm{SCET}_{\mathrm{II}}$ boundaries, the effective field theory involves additional collinear-soft modes. This type of mode was introduced in a different context in ref. [52], and has led us to also refer to our effective theory as $\mathrm{SCET}_{+}$. Since we are considering different observables than ref. [52], there are of course important differences, which will be discussed in section 2.1. We now comment on the theoretical description relevant for each region of phase space in the $\left(p_{T}, \mathcal{T}\right)$ plane.

\section{- Fixed Order: $p_{T}, \mathcal{T} \sim Q$}

When $p_{T}$ and $\mathcal{T}$ are parametrically of the same size as the hard scale $Q^{2}=p_{Z}^{2} \sim m_{Z}^{2}$, resummation is not necessary and a fixed-order calculation suffices.

- $\mathrm{SCET}_{\mathrm{I}}: p_{T} \sim Q^{1 / 2} \mathcal{T}^{1 / 2}$

This case was discussed in ref. [53]. The collinear and soft modes, shown in the left panel of figure 2, interact. The $\mathrm{SCET}_{\mathrm{I}}$ scale hierarchy implies that the soft radiation contributes only to $\mathcal{T}$ (its contribution to $p_{T}$ is power suppressed), whereas 
the collinear radiation contributes both to the $p_{T}$ and the $\mathcal{T}$ measurement. This collinear radiation is described by fully-unintegrated parton distribution functions (PDFs) [53-56], which depend on all momentum components of the colliding parton. By contrast, the standard PDFs only depend on the momentum fraction $x$.

- $\operatorname{SCET}_{+}: p_{T} \sim Q^{1-r} \mathcal{T}^{r}$ with $1 / 2<r<1$

As $p_{T}$ is lowered, the collinear modes can no longer interact with the soft mode. They "split off" collinear-soft modes that do interact with the soft modes, see figure 2. (To have a distinct mode contribution requires sufficient distance from the $\mathrm{SCET}_{\mathrm{I}}$ and $\mathrm{SCET}_{\mathrm{II}}$ boundaries.) In this scenario, the collinear radiation only contributes to $p_{T}$, the soft radiation only to $\mathcal{T}$, and the collinear-soft radiation enters in both measurements. The SCET + power counting will be given below in table 1 .

- $\operatorname{SCET}_{\mathrm{II}}: p_{T} \sim \mathcal{T}$

As $p_{T}$ is reduced further, the soft mode "absorbs" the two collinear-soft modes. In the resulting theory there are no interactions between the collinear and the soft modes, as shown in the left panel of figure 2. The collinear radiation, which in the SCET II case is described by transverse-momentum dependent (TMD) PDFs, only affects $p_{T}$, whereas now the soft mode contributes to both measurements.

- $Z$ +forward jet: $p_{T} \gg Q^{1 / 2} \mathcal{T}^{1 / 2}$

As $p_{T}$ exceeds this bound, the QCD radiation becomes (much) more energetic than the invariant mass $Q$ of the $Z$ boson. This cannot be described as initial-state radiation, but rather as $Z$ production in association with an energetic forward jet.

- Terra incognita: $p_{T} \ll \mathcal{T}$

Unlike the previous regions, the cross section no longer receives a contribution from a single emission. There is a small NNLO contribution from the region of phase space where the two emissions are (almost) back-to-back in the transverse plane. In double parton scattering (DPS) the production of the $Z$ and the two jets are (largerly) independent of each other, causing the jets to naturally be back-to-back. ${ }^{1}$ The contribution from DPS is therefore also important. As the proper method for combining single and double parton scattering is still under debate [59-64], we leave this for future work.

In this paper, we also show how to combine the $\mathrm{SCET}_{\mathrm{I}}, \mathrm{SCET}_{+}$and $\mathrm{SCET}_{\mathrm{II}}$ regions to achieve NNLL resummation throughout. The corresponding next-to-leading order cross section is calculated, providing a check of our results.

In most earlier studies of multi-dimensional observables in SCET, such as refs. [65, 66], the measurements concerned different regions of phase space (hemispheres, jets, etc.). There, resummation is achieved by assuming a single parametric relation between the observables, to avoid so-called non-global logarithms [67, 68]. In ref. [34] the two boundary theories for the measurement of two angularities on a single jet were identified. There an

\footnotetext{
${ }^{1}$ This feature is exploited to extract DPS experimentally, see e.g. refs. [57, 58].
} 

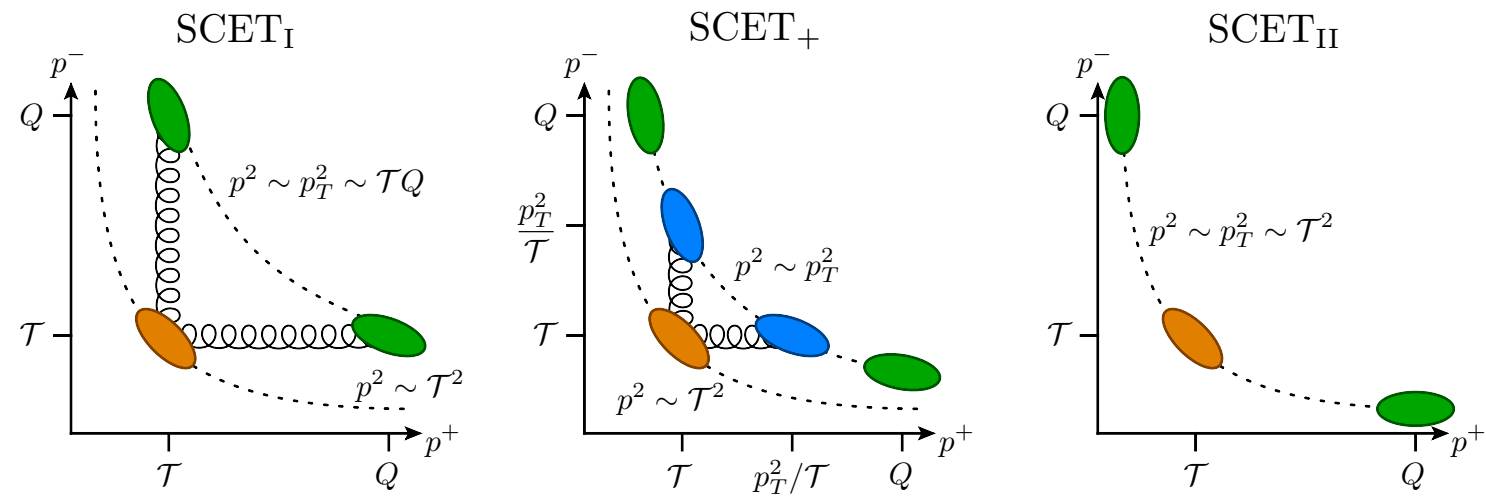

Figure 2. The modes in $\mathrm{SCET}_{\mathrm{I}}, \mathrm{SCET}_{+}$and $\mathrm{SCET}_{\mathrm{II}}$ : collinear (green), collinear-soft (blue) and soft (orange). Interactions between modes in the effective theory are shown with wiggly lines. These are removed by the decoupling transformations in eq. (2.7).

interpolating function across the intermediate region was derived, by requiring it to be continuous and have a continuous derivative at the boundaries. We revisit their NLL results and find a discrepancy at $\mathcal{O}\left(\alpha_{s}^{2}\right)$ in the bulk. It is worth mentioning that in this case both boundaries involve $\mathrm{SCET}_{\mathrm{I}}$-type theories, to which our framework can be applied as well.

The paper is structured as follows. In section 2.1 we introduce $\mathrm{SCET}_{+}$, perform the matching of QCD onto $\mathrm{SCET}_{+}$currents, and comment on the (dis)similarities with the theory introduced in ref. [52]. Section 2.2 contains the factorization formulae for the DrellYan cross section with a simultaneous measurement of $p_{T}$ and $\mathcal{T}$ in the $\mathrm{SCET}_{\mathrm{I}}, \mathrm{SCET}_{+}$ and $\mathrm{SCET}_{\mathrm{II}}$ regions of phase space, as well as the field-theoretic definitions of the matrix elements involved. We calculate/collect all the ingredients necessary to achieve NNLL accuracy in section 3 and discuss the (all-order) matching of $\mathrm{SCET}_{\mathrm{I}}, \mathrm{SCET}_{+}$and $\mathrm{SCET}_{\mathrm{II}}$ in section 4. The corresponding NLO cross section is calculated in section 5, providing a verification of our resummed predictions. In section 6 we calculate the double angularity measurement on a single jet and compare with ref. [34]. Conclusions and outlook are presented in section 7 .

\section{Factorization}

\subsection{Effective theory for the region between $\mathrm{SCET}_{\mathrm{I}}$ and $\mathrm{SCET}_{\mathrm{II}}$ boundaries}

Soft-Collinear Effective Theory (SCET) [48-51] describes the collinear and soft limits of QCD. For a pedagogical introduction see e.g. refs. [69, 70]. SCET captures QCD in the infrared regime up to corrections that are suppressed by powers of the SCET expansion parameter $\lambda \ll 1$, in exchange for enabling the resummation of large logarithms of $\lambda$. As discussed in section 1 , both the process and measurement determine which modes give the leading contributions to the cross section in a specific kinematic regime. In figure 2 we summarize the scalings and interactions between different degrees of freedom leading to the physical picture in section 1 . These modes need to be well-separated, in order for $\lambda$ to be small. The decoupling of modes in the SCET Lagrangian (at leading power) allows one to 


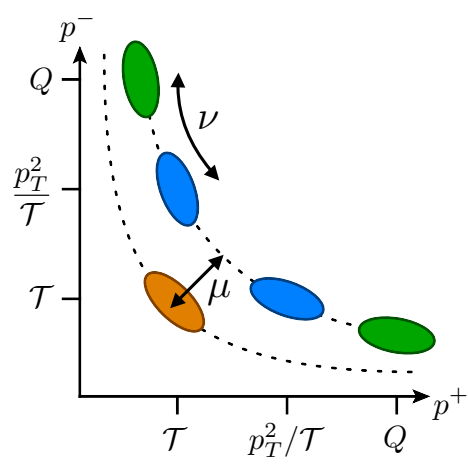

Figure 3. The $\mu$-evolution resums double logarithms from separations in virtuality (between hyperbolae), while the $\nu$-evolution resums single logarithms related to separations in rapidity (along hyperbolae). The collinear, collinear-soft and soft modes are depicted in green, blue and orange, respectively.

\begin{tabular}{|l|rr|}
\hline Mode: & Scaling $(-,+, \perp)$ & \\
\hline$n$-collinear & $Q\left(1, \lambda^{2 r}, \lambda^{r}\right)$ & $\sim\left(Q, p_{T}^{2} / Q, p_{T}\right)$ \\
$\bar{n}$-collinear & $Q\left(\lambda^{2 r}, 1, \lambda^{r}\right)$ & $\sim\left(p_{T}^{2} / Q, Q, p_{T}\right)$ \\
$n$-collinear-soft & $Q\left(\lambda^{2 r-1}, \lambda, \lambda^{r}\right)$ & $\sim\left(p_{T}^{2} / \mathcal{T}, \mathcal{T}, p_{T}\right)$ \\
$\bar{n}$-collinear-soft & $Q\left(\lambda, \lambda^{2 r-1}, \lambda^{r}\right)$ & $\sim\left(\mathcal{T}, p_{T}^{2} / \mathcal{T}, p_{T}\right)$ \\
soft & $Q(\lambda, \lambda, \lambda)$ & $\sim(\mathcal{T}, \mathcal{T}, \mathcal{T})$ \\
\hline
\end{tabular}

Table 1. Modes and power counting in $\operatorname{SCET}_{+}$with $\lambda \sim \mathcal{T} / Q \sim\left(p_{T} / Q\right)^{1 / r}$.

factorize multi-scale cross sections into products (or convolutions) of single-scale functions for each mode. At its natural scale, each of these function contains no large logarithms. By applying the renormalization group (RG) evolution from these natural scales to a common scale $\mu$, we achieve resummation of logarithms of $\lambda$ in the cross section. For modes that are not separated in virtuality but only in rapidity, we will sum the corresponding single logarithms through the $\nu$-evolution of the rapidity renormalization group [71, 72]. ${ }^{2}$ Pictorially, the $\mu$-evolution sums logarithms related to the separation between the mass hyperbolae of the modes, whereas the $\nu$-evolution sums the logarithms related to the separation along them, see figure 3 .

We will now discuss SCET $_{+}$in some detail, focussing on modes, matching of QCD onto $\mathrm{SCET}_{+}$and factorization. We refrain from performing a full formal construction of the effective theory. Factorization means there are no interactions between the various modes, and each mode is described by a (boosted) copy of QCD. In particular, one can use the standard QCD Feynman rules (rather than e.g. the collinear effective Lagrangian of ref. [49]) to carry out the computations for each sector.

The measurement of beam thrust $\mathcal{T}$ and transverse momentum $p_{T}$, with $p_{T} \sim Q^{1-r} \mathcal{T}^{r}$ and $1 / 2<r<1,{ }^{3}$ suggests that the relevant modes are those listed in table 1 and shown

\footnotetext{
${ }^{2}$ For alternative approaches to rapidity resummation in SCET, see e.g. refs. [14, 73].

${ }^{3}$ Note that our analysis is independent of the parameter $r$, as is clear from the second way of writing the modes in table 1 . However, we prefer to use a single power counting parameter $\lambda$.
} 
in the center panel of figure 2 , with power counting parameter

$$
\lambda \sim \frac{\mathcal{T}}{Q} \sim\left(\frac{p_{T}}{Q}\right)^{1 / r} .
$$

A collinear mode only affects the $p_{T}$-distribution, as the contribution to $\mathcal{T}$ from its small light-cone component is power suppressed. Similarly, a soft mode only contributes to $\mathcal{T}$, whereas the collinear-soft modes contributes to both measurements. These on-shell modes are uniquely specified by these features. Of course, additional (redundant) modes may be included, as long as the double counting is removed (for example by an appropriate zero-bin subtraction [74]). As usual, we will assume the cancellation of (off-shell) Glauber modes. These account for initial-state hadron-hadron interactions taking place before the collision, which would ruin factorization [75]. This cancellation has only been rigorously proven for inclusive Drell-Yan [76], and could be spoiled due to our $p_{T}$ and $\mathcal{T}$ measurements [77].

The QCD quark and gluon fields are decomposed into several SCET fields which scale differently with respect to the expansion parameter $\lambda$. By matching quark currents from QCD onto $\mathrm{SCET}_{+}$we obtain

$$
\bar{\Psi} \Gamma \Psi=C\left(Q^{2}, \mu\right) \bar{\xi}_{\bar{n}} W_{\bar{n}} S_{\bar{n}}^{\dagger} X_{\bar{n}}^{\dagger} V_{\bar{n}} \Gamma V_{n}^{\dagger} X_{n} S_{n} W_{n}^{\dagger} \xi_{n} .
$$

The matching coefficient $C\left(Q^{2}, \mu\right)$ captures the effect of hard virtual gluon exchanges not present in the effective theory. In eq. (2.2), $\xi_{n}$ and $\bar{\xi}_{\bar{n}}$ are the fields for collinear (anti-) quarks moving in the $n(\bar{n})$ direction and $\Gamma$ denotes a generic Dirac structure. The Wilson line $W_{n}$ arises from $n$-collinear gluons emitted by $\bar{\Psi}$ (which itself is $\bar{n}$-collinear) [50]

$$
W_{n}=P \exp \left[\mathrm{i} g \int_{-\infty}^{0} \mathrm{~d} u \bar{n} \cdot A_{n}(u \bar{n})\right] .
$$

The Wilson line $V_{n}$ is its direct analog for $n$-collinear-soft gluons (obtained by replacing $\left.A_{n} \rightarrow A_{n c s}\right)$. Soft gluons emitted by $\Psi$ are summed into the Wilson line $S_{n}[51]$

$$
S_{n}=P \exp \left[\mathrm{i} g \int_{-\infty}^{0} \mathrm{~d} u n \cdot A_{s}(u n)\right],
$$

and the analog for $n$-collinear-soft gluons is $X_{n}$.

To fix the ordering of Wilson lines, we exploit gauge invariance of $\mathrm{SCET}_{+}$. In order to preserve the scaling of the fields, separate collinear, collinear-soft and soft gauge transformations have to be introduced, see e.g. refs. [51, 52]. Only the $n$-collinear fields transform under $n$-collinear gauge transformations. The other fields are taken far off-shell and are thus unable to resolve the local change induced by this gauge transformation. This causes $W_{n}^{\dagger} \xi_{n}$ and $\bar{\xi}_{\bar{n}} W_{\bar{n}}$ to be grouped together. Under a $n$-collinear-soft gauge transformation $U_{n c s}$

$$
\begin{array}{rlrlrl}
W_{n}^{\dagger} \xi_{n} & \rightarrow W_{n}^{\dagger} \xi_{n}, & S_{n} \rightarrow S_{n}, & V_{n} \rightarrow U_{n c s} V_{n}, & & X_{n} \rightarrow U_{n c s} X_{n}, \\
\bar{\xi}_{\bar{n}} W_{\bar{n}} \rightarrow \bar{\xi}_{\bar{n}} W_{\bar{n}}, & S_{\bar{n}} \rightarrow S_{\bar{n}}, & V_{\bar{n}} \rightarrow V_{\bar{n}}, & X_{\bar{n}} \rightarrow X_{\bar{n}},
\end{array}
$$

which groups $V_{n}^{\dagger} X_{n}$ together. Similarly, $X_{\bar{n}}^{\dagger} V_{\bar{n}}$ must be grouped together by $\bar{n}$-collinear-soft gauge invariance. The effect of a soft gauge transformation $U_{s}$ is given by

$$
\begin{array}{llll}
W_{n}^{\dagger} \xi_{n} \rightarrow W_{n}^{\dagger} \xi_{n}, & S_{n} \rightarrow U_{s} S_{n}, & V_{n} \rightarrow U_{s} V_{n} U_{s}^{\dagger}, & X_{n} \rightarrow U_{s} X_{n} U_{s}^{\dagger}, \\
\bar{\xi}_{\bar{n}} W_{\bar{n}} \rightarrow \bar{\xi}_{\bar{n}} W_{\bar{n}}, & S_{\bar{n}} \rightarrow U_{s} S_{\bar{n}}, & V_{\bar{n}} \rightarrow U_{s} V_{\bar{n}} U_{s}^{\dagger}, & X_{\bar{n}} \rightarrow U_{s} X_{\bar{n}} U_{s}^{\dagger} .
\end{array}
$$


The soft gluon field acts as smooth background for collinear-soft modes, implying that the effect of a soft gauge transformation on collinear-soft modes is similar to a global color rotation. This almost fixes the ordering in eq. (2.2). There are still a few other possibilities that satisfy the constraints from gauge invariance, such as $\bar{\xi}_{\bar{n}} W_{\bar{n}} S_{\bar{n}}^{\dagger} V_{n}^{\dagger} X_{n} \Gamma X_{\bar{n}}^{\dagger} V_{\bar{n}} S_{n} W_{n}^{\dagger} \xi_{n}$. However, these can be ruled out by considering the tree-level matching of QCD onto SCET + .

At this point the soft fields still interact with the collinear-soft fields, as indicated in the middle panel of figure 2. By performing the analog of the BPS field redefinition [51], we decouple the soft fields from the collinear-soft fields,

$$
\begin{array}{ll}
V_{n} \rightarrow S_{n} V_{n} S_{n}^{\dagger}, & X_{n} \rightarrow S_{n} X_{n} S_{n}^{\dagger}, \\
V_{\bar{n}} \rightarrow S_{\bar{n}} V_{\bar{n}} S_{\bar{n}}^{\dagger}, & X_{\bar{n}} \rightarrow S_{\bar{n}} X_{\bar{n}} S_{\bar{n}}^{\dagger} .
\end{array}
$$

This leads to

$$
\bar{\Psi} \Gamma \Psi=C\left(Q^{2}, \mu\right) \bar{\xi}_{\bar{n}} W_{\bar{n}} X_{\bar{n}}^{\dagger} V_{\bar{n}} S_{\bar{n}}^{\dagger} \Gamma S_{n} V_{n}^{\dagger} X_{n} W_{n}^{\dagger} \xi_{n}
$$

The various modes in this matching equation no longer interact and the derivation of factorization formulae now follows the standard procedure in SCET. In particular, establishing factorization to all orders in $\alpha_{s}$ requires decoupling of the different modes in the Lagrangian, for which we refer to ref. [52].

One expects that this matching receives power corrections of the size $\lambda^{2 r-1} \sim p_{T}^{2} /(Q \mathcal{T})$ and $\lambda^{2-2 r} \sim \mathcal{T}^{2} / p_{T}^{2}$, which measure the distance from the respective $\mathrm{SCET}_{\mathrm{I}}$ and $\mathrm{SCET}_{\mathrm{II}}$ boundary regions of phase space. In our NLO calculation in section 5 we find corrections of the first type but not of the second. However, we expect that this will no longer be the case at higher orders.

Finally, we briefly comment on the (dis)similarities of our theory with the $\mathrm{SCET}_{+}$ introduced in ref. [52]. In that paper the dijet invariant mass $\left(m_{j_{1} j_{2}}\right)$ distribution for nearby jets is calculated, with the hierarchy $m_{j_{1}}, m_{j_{2}} \ll m_{j_{1} j_{2}} \ll Q$. Their collinear-soft modes can resolve the two nearby jets, whereas the soft modes do not, and the collinear modes are restricted to the individual jets. Their factorization theorem involves convolutions through the small collinear light-cone component. Since we consider different type of observables, our convolutions of collinear-soft modes with either collinear or soft radiation have a different structure. The matching in ref. [52] was (also) performed in two steps, where in the first step the two nearby jets are not resolved from each other. Nevertheless, the similarities between the modes and Wilson lines in our and their approach seemed sufficient to us to adopt the same name for our effective theory.

\subsection{Factorization formulae}

We now discuss SCET factorization formulae for Drell-Yan cross sections that are differential both in $\mathcal{T}$ and $p_{T}$, both at the $\mathrm{SCET}_{\mathrm{I}}$ and $\mathrm{SCET}_{\mathrm{II}}$ phase space boundaries and in the $\mathrm{SCET}_{+}$"bulk". In Drell-Yan production, $p p \rightarrow Z / \gamma^{*} \rightarrow \ell^{+} \ell^{-}$, the lepton pair has a large invariant mass $Q$. A proof of factorization at leading power in $\Lambda_{\mathrm{QCD}} / Q$ has been given by Collins, Soper and Sterman [9], for any value of the transverse momentum $p_{T}$ of the lepton pair, namely for both $p_{T} \sim Q$ and $p_{T} \ll Q$. Here we impose in addition a 
veto on hard central jets through a cut on beam thrust $\mathcal{T}$ in the center-of-mass frame of the $p p$ collision [1], see eq. (1.1). We consider different kinematic regimes for $p_{T}$ and $\mathcal{T}$, as discussed in the introduction. We will not perform the joint resummation of threshold logarithms that becomes important as $Q$ approaches the total CM energy $E_{\mathrm{cm}}$ [78].

If $\Lambda_{\mathrm{QCD}} \ll p_{T} \sim(\mathcal{T} Q)^{1 / 2} \ll Q\left(\mathrm{SCET}_{\mathrm{I}}\right.$ case), we have the following leading-power factorization formula $[46,53]$

$$
\begin{aligned}
\frac{\mathrm{d}^{4} \sigma}{\mathrm{d} Q^{2} \mathrm{~d} Y \mathrm{~d} p_{T}^{2} \mathrm{~d} \mathcal{T}}= & \sum_{q} \hat{\sigma}_{q}^{0} H\left(Q^{2}, \mu\right) \int \mathrm{d} t_{1} \mathrm{~d} t_{2} \int \mathrm{d}^{2} \vec{k}_{1 \perp} \mathrm{d}^{2} \vec{k}_{2 \perp} \int \mathrm{d} k^{+} S\left(k^{+}, \mu\right) \\
& \times\left[B_{q}\left(t_{1}, x_{1}, \vec{k}_{1 \perp}, \mu\right) B_{\bar{q}}\left(t_{2}, x_{2}, \vec{k}_{2 \perp}, \mu\right)+(q \leftrightarrow \bar{q})\right] \\
& \times \delta\left(\mathcal{T}-\frac{e^{-Y} t_{1}+e^{Y} t_{2}}{Q}-k^{+}\right) \delta\left(p_{T}^{2}-\left|\vec{k}_{1 \perp}+\vec{k}_{2 \perp}\right|^{2}\right),
\end{aligned}
$$

whose ingredients we will describe below. The sum extends over the various quark flavors, $Y$ is the total rapidity of the leptons, and the momentum fractions of the colliding partons are

$$
x_{1}=\frac{Q}{E_{\mathrm{cm}}} e^{Y}, \quad x_{2}=\frac{Q}{E_{\mathrm{cm}}} e^{-Y} .
$$

The quantities $e^{-Y} t_{1} / Q, e^{Y} t_{2} / Q$ and $k^{+}$in eq. (2.9) are the contributions to $\mathcal{T}$ from the $n$ collinear, $\bar{n}$-collinear and soft radiation. For $n$-collinear radiation, we always have $p_{i}^{+}<p_{i}^{-}$, for $\bar{n}$-collinear radiation, $p_{i}^{+}>p_{i}^{-}$, whereas the soft radiation can go into both hemispheres $\left(p_{i}^{+}<p_{i}^{-}\right.$and $\left.p_{i}^{+}>p_{i}^{-}\right)$.

At leading order in the electroweak interactions,

$$
\hat{\sigma}_{q}^{0}=\frac{4 \pi \alpha_{\mathrm{em}}^{2}}{9 Q^{2} E_{\mathrm{cm}}^{2}}\left[Q_{q}^{2}+\frac{\left(v_{q}^{2}+a_{q}^{2}\right)\left(v_{\ell}^{2}+a_{\ell}^{2}\right)-2 Q_{q} v_{q} v_{\ell}\left(1-m_{Z}^{2} / Q^{2}\right)}{\left(1-m_{Z}^{2} / Q^{2}\right)^{2}+m_{Z}^{2} \Gamma_{Z}^{2} / Q^{4}}\right],
$$

where $Q_{q}$ is the quark charge in units of $|e|, v_{\ell, q}$ and $a_{\ell, q}$ are the standard vector and axial couplings of the leptons and quarks, and $m_{Z}$ and $\Gamma_{Z}$ are the mass and width of the $Z$ boson.

The hard function $H\left(Q^{2}\right)$ is the square of the Wilson coefficient $C\left(Q^{2}\right)$ for the matching of QCD onto SCET vector and axial quark currents ${ }^{4}$

$$
H\left(Q^{2}, \mu\right)=\left|C\left(Q^{2}, \mu\right)\right|^{2} .
$$

It does not depend on $p_{T}$, since we only consider $p_{T} \ll Q .{ }^{5} \quad$ Since lepton masses are neglected, there is no contribution from gluon operators in the matching of the (axial) currents [46]. The gluon PDF only appears through its contribution to the quark beam function, see eq. (3.2).

Due to the $\mathrm{SCET}_{\mathrm{I}}$ hierarchy of scales, the effect of soft radiation on the $p_{T}$-distribution is power suppressed, so only the fully-unintegrated (FU) PDFs account for the recoil of the energetic initial-state radiation against the final-state leptons. Because we consider

\footnotetext{
${ }^{4}$ As compared to eq. (2.2), in $\mathrm{SCET}_{\mathrm{I}}$ only collinear and (ultra-)soft Wilson lines enter the matching.

${ }^{5}$ The leptonic tensor in the Drell-Yan process does not depend on $p_{T}$ at leading order.
} 
perturbative $p_{T}, \mathcal{T} \gg \Lambda_{\mathrm{QCD}}$, we will refer to these as $\mathrm{FU}$ beam functions in the following. At the bare level, these are defined as the following proton matrix element of collinear fields [53]

$$
B_{q}\left(t, x, \vec{k}_{\perp}\right)=\left\langle p_{n}\left(p^{-}\right)\left|\bar{\chi}_{n}(0) \frac{\not h}{2}\left[\delta\left(k^{-}-p^{-}+\mathbf{P}^{-}\right) \delta\left(t-k^{-} \mathbf{P}^{+}\right) \delta^{2}\left(\vec{k}_{\perp}-\overrightarrow{\mathbf{P}}_{\perp}\right) \chi_{n}(0)\right]\right| p_{n}\left(p^{-}\right)\right\rangle .
$$

The light-like vector $n^{\mu}$ is along the direction of the incoming proton (i.e. $p^{\mu}=E_{\mathrm{cm}} n^{\mu} / 2$ ) and the operator $\mathbf{P}$ returns the momentum of the intermediate state. ${ }^{6}$ By boost invariance along the $n$-direction, these functions only depend on the momentum fraction $x=k^{-} / p^{-}$, the transverse virtuality $-t=k^{-} k^{+}$of the colliding parton, and the transverse momentum $\vec{k}_{\perp}[53,79]$.

The (ultra-)soft radiation is described by the beam thrust soft function $S(k)$ [46]. This is given in terms of a soft Wilson-line correlator as

$$
S\left(k^{+}\right)=\frac{1}{N_{c}}\left\langle 0\left|\operatorname{Tr}\left[\overline{\mathbf{T}}\left(S_{n}^{\dagger}(0) S_{\bar{n}}(0)\right) \delta\left(k^{+}-\mathbf{P}_{1}^{+}-\mathbf{P}_{2}^{-}\right) \mathbf{T}\left(S_{\bar{n}}^{\dagger}(0) S_{n}(0)\right)\right]\right| 0\right\rangle,
$$

where $(\overline{\mathbf{T}}) \mathbf{T}$ denotes (anti)time ordering and the operator $\mathbf{P}_{1}\left(\mathbf{P}_{2}\right)$ gives the momentum of the soft radiation going into the hemisphere defined by $p_{i}^{+}<p_{i}^{-}\left(p_{i}^{+}>p_{i}^{-}\right)$.

In the region of phase space described by $\operatorname{SCET}_{+}\left(\Lambda_{\mathrm{QCD}} \ll \mathcal{T} \ll p_{T} \ll(\mathcal{T} Q)^{1 / 2} \ll Q\right)$,

$$
\begin{aligned}
\frac{\mathrm{d}^{4} \sigma}{\mathrm{d} Q^{2} \mathrm{~d} Y \mathrm{~d} p_{T}^{2} \mathrm{~d} \mathcal{T}}= & \sum_{q} \hat{\sigma}_{q}^{0} H\left(Q^{2}, \mu\right) \int \mathrm{d}^{2} \vec{k}_{1 \perp} \mathrm{d}^{2} \vec{k}_{2 \perp} \mathrm{d}^{2} \vec{k}_{1 \perp}^{\mathrm{cs}} \mathrm{d}^{2} \vec{k}_{2 \perp}^{\mathrm{cs}} \int \mathrm{d} k_{1}^{+} \mathrm{d} k_{2}^{+} \mathrm{d} k^{+} S\left(k^{+}, \mu\right) \\
& \times B_{q}\left(x_{1}, \vec{k}_{1 \perp}, \mu, \nu\right) B_{\bar{q}}\left(x_{2}, \vec{k}_{2 \perp}, \mu, \nu\right) \mathscr{S}\left(k_{1}^{+}, \vec{k}_{1 \perp}^{\mathrm{cs}}, \mu, \nu\right) \mathscr{S}\left(k_{2}^{+}, \vec{k}_{2 \perp}^{\mathrm{cs}}, \mu, \nu\right) \\
& \times \delta\left(\mathcal{T}-k_{1}^{+}-k_{2}^{+}-k^{+}\right) \delta\left(p_{T}^{2}-\left|\vec{k}_{1 \perp}+\vec{k}_{2 \perp}+\vec{k}_{1 \perp}^{\mathrm{cs}}+\vec{k}_{2 \perp}^{\mathrm{cs}}\right|^{2}\right)+(q \leftrightarrow \bar{q}) .
\end{aligned}
$$

The contribution from collinear radiation is now encoded in TMD beam functions,

$$
B_{q}\left(x, \vec{k}_{\perp}\right)=\left\langle p_{n}\left(p^{-}\right)\left|\bar{\chi}_{n}(0) \frac{\not h}{2}\left[\delta\left(k^{-}-p^{-}+\mathbf{P}^{-}\right) \delta^{2}\left(\vec{k}_{\perp}-\overrightarrow{\mathbf{P}}_{\perp}\right) \chi_{n}(0)\right]\right| p_{n}\left(p^{-}\right)\right\rangle
$$

Their naive definition using dimensional regularization is known to suffer from light-cone singularities (rapidity divergences), which we regulate following refs. [71, 72]. There are separate but identical collinear-soft functions for the $n$ and $\bar{n}$ direction,

$$
\begin{aligned}
\mathscr{S}\left(k^{+}, \vec{k}_{\perp}\right) & =\frac{1}{N_{c}}\left\langle 0\left|\operatorname{Tr}\left[\overline{\mathbf{T}}\left(X_{n}^{\dagger}(0) V_{n}(0)\right) \delta\left(k^{+}-\mathbf{P}^{+}\right) \delta^{2}\left(\vec{k}_{\perp}-\overrightarrow{\mathbf{P}}_{\perp}\right) \mathbf{T}\left(V_{n}^{\dagger}(0) X_{n}(0)\right)\right]\right| 0\right\rangle, \\
& =\frac{1}{N_{c}}\left\langle 0\left|\operatorname{Tr}\left[\overline{\mathbf{T}}\left(V_{\bar{n}}^{\dagger}(0) X_{\bar{n}}(0)\right) \delta\left(k^{+}-\mathbf{P}^{-}\right) \delta^{2}\left(\vec{k}_{\perp}-\overrightarrow{\mathbf{P}}_{\perp}\right) \mathbf{T}\left(X_{\bar{n}}^{\dagger}(0) V_{\bar{n}}(0)\right)\right]\right| 0\right\rangle,
\end{aligned}
$$

which are also affected by rapidity divergences.

For the hierarchy $\Lambda_{\mathrm{QCD}} \ll p_{T} \sim \mathcal{T} \ll Q$, soft modes have the same virtuality and transverse momentum as the collinear ones, and contribute both to $\mathcal{T}$ and $p_{T}$ measurements. The corresponding $\mathrm{SCET}_{\mathrm{II}}$ factorization theorem has the form

\footnotetext{
${ }^{6}$ We can avoid using the label-momentum formalism employed in e.g. refs. [53, 79] since after factorization the collinear sector is simply a boosted copy of QCD.
} 


$$
\begin{aligned}
\frac{\mathrm{d}^{4} \sigma}{\mathrm{d} Q^{2} \mathrm{~d} Y \mathrm{~d} p_{T}^{2} \mathrm{~d} \mathcal{T}}= & \sum_{q} \hat{\sigma}_{q}^{0} H\left(Q^{2}, \mu\right) \int \mathrm{d}^{2} \vec{k}_{1 \perp} \mathrm{d}^{2} \vec{k}_{2 \perp} \mathrm{d}^{2} \vec{k}_{\perp} \int \mathrm{d} k^{+} \delta\left(p_{T}^{2}-\left|\vec{k}_{1 \perp}+\vec{k}_{2 \perp}+\vec{k}_{\perp}\right|^{2}\right) \delta\left(\mathcal{T}-k^{+}\right) \\
& \times\left[B_{q}\left(x_{1}, \vec{k}_{1 \perp}, \mu, \nu\right) B_{\bar{q}}\left(x_{2}, \vec{k}_{2 \perp}, \mu, \nu\right)+(q \leftrightarrow \bar{q})\right] S\left(k^{+}, \vec{k}_{\perp}, \mu, \nu\right)
\end{aligned}
$$

The new ingredient is given by the FU soft function, which is defined as

$$
S\left(k^{+}, \vec{k}_{\perp}\right)=\frac{1}{N_{c}}\left\langle 0\left|\operatorname{Tr}\left[\overline{\mathbf{T}}\left(S_{n}^{\dagger}(0) S_{\bar{n}}(0)\right) \delta\left(k^{+}-\mathbf{P}_{1}^{+}-\mathbf{P}_{2}^{-}\right) \delta^{2}\left(\vec{k}_{\perp}-\overrightarrow{\mathbf{P}}_{\perp}\right) \mathbf{T}\left(S_{\bar{n}}^{\dagger}(0) S_{n}(0)\right)\right]\right| 0\right\rangle .
$$

It is natural to ask to what extent our approach can be used to calculate non-global logarithms, which arise when different restrictions are applied to distinct regions of phase space $[67,68]$. If instead of the transverse momentum of the $Z$ boson one measures the $p_{T, \text { ISR }}$ of the initial-state radiation that recoils against it, we could e.g. restrict ourselves to the ISR in one hemisphere. In this case the factorization theorem in the region of phase space described by $\mathrm{SCET}_{+}$is simply modified to

$$
\begin{aligned}
\frac{\mathrm{d}^{4} \sigma}{\mathrm{d} Q^{2} \mathrm{~d} Y \mathrm{~d} p_{T, \text { ISR }}^{2} \mathrm{~d} \mathcal{T}}= & \sum_{q} \hat{\sigma}_{q}^{0} H\left(Q^{2}, \mu\right) \int \mathrm{d} t_{2} \int \mathrm{d}^{2} \vec{k}_{1 \perp} \mathrm{d}^{2} \vec{k}_{1 \perp}^{\mathrm{cs}} \int \mathrm{d} k_{1}^{+} \mathrm{d} k^{+} S\left(k^{+}, \mu\right) \\
& \times B_{q}\left(x_{1}, \vec{k}_{1 \perp}, \mu, \nu\right) B_{\bar{q}}\left(t_{2}, x_{2}, \mu\right) \mathscr{S}\left(k_{1}^{+}, \vec{k}_{1 \perp}^{\mathrm{cs}}, \mu, \nu\right) \\
& \times \delta\left(\mathcal{T}-k_{1}^{+}-\frac{e^{Y} t_{2}}{Q}-k^{+}\right) \delta\left(p_{T, \mathrm{ISR}}^{2}-\left|\vec{k}_{1 \perp}+\vec{k}_{1 \perp}^{\mathrm{cs}}\right|^{2}\right)+(q \leftrightarrow \bar{q}) .
\end{aligned}
$$

However, this does not address the problem arising when the soft function contains multiple scales (see for example [80-82]), which occurs when e.g. the beam thrust measurement is restricted to one hemisphere.

\section{Ingredients at NNLL}

In this section we collect the expressions for the ingredients entering the factorization formulae in section 2.2, to the accuracy needed for NNLL resummations: the hard function at one loop is discussed in section 3.1, the FU and TMD beam function in section 3.2, the FU and beam thrust soft function in section 3.3 and the collinear-soft function in section 3.4. The FU soft function and collinear-soft function are calculated for the first time. RG equations and anomalous dimensions for NNLL resummation are given in section 3.5 and appendix B. The anomalous dimensions of the collinear-soft function and FU soft function satisfy the consistency requirement imposed by the $\mu$ and $\nu$ independence of the factorized cross sections in eqs. (2.15) and (2.18). In section 3.6 we combine these ingredients to obtain a compact expression for the NLL cross section.

\subsection{Hard function}

The one-loop Wilson coefficient $C\left(Q^{2}, \mu\right)$ from matching the quark current in QCD onto SCET was computed in refs. $[83,84]$. Here $Q^{2}$ is the square of the partonic center of mass 
energy. The matching is the same for $\mathrm{SCET}_{\mathrm{I}}, \mathrm{SCET}_{+}$and $\mathrm{SCET}_{\mathrm{II}}$, because all effective field theory diagrams are scaleless and vanish in dimensional regularization. At one loop,

$$
H\left(Q^{2}, \mu\right)=\left|C\left(Q^{2}, \mu\right)\right|^{2}=1+\frac{\alpha_{s} C_{F}}{2 \pi}\left[-\ln ^{2}\left(\frac{Q^{2}}{\mu^{2}}\right)+3 \ln \left(\frac{Q^{2}}{\mu^{2}}\right)-8+\frac{7 \pi^{2}}{6}\right] .
$$

\subsection{Beam functions}

The FU beam function was defined in eq. (2.13), and its arguments $t$ and $\vec{k}_{\perp}^{2}$ are restricted to be of the same parametric size. As we assume that these scales are perturbative, the FU beam function can be matched onto PDFs [16, 46, 53, 79]

$$
B_{q}\left(t, x, \vec{k}_{\perp}, \mu\right)=\sum_{j=u, \bar{u}, d, g \ldots . .} \int_{x}^{1} \frac{\mathrm{d} x^{\prime}}{x^{\prime}} \mathcal{I}_{q j}\left(t, \frac{x}{x^{\prime}}, \vec{k}_{\perp}, \mu\right) f_{j}\left(x^{\prime}, \mu\right)\left[1+\mathcal{O}\left(\frac{\Lambda_{\mathrm{QCD}}^{2}}{t}, \frac{\Lambda_{\mathrm{QCD}}^{2}}{\vec{k}_{\perp}^{2}}\right)\right] .
$$

Because of the kinematic bound $\vec{k}_{\perp}^{2} \leq(1-x) t / x$ (see eq. (1.1) of ref. [53]), the renormalization is the same as the standard beam function and

$$
\int \mathrm{d}^{2} \vec{k}_{\perp} B_{q}\left(t, x, \vec{k}_{\perp}, \mu\right)=B_{q}(t, x, \mu) .
$$

Up to NLO, the matching coefficients in eq. (3.2) are [53]

$$
\begin{aligned}
\mathcal{I}_{q q}^{(0)}\left(t, x, \vec{k}_{\perp}, \mu\right)= & \delta(t) \delta(1-x) \delta^{2}\left(\vec{k}_{\perp}\right) \\
\mathcal{I}_{q g}^{(0)}\left(t, x, \vec{k}_{\perp}, \mu\right)= & 0 \\
\mathcal{I}_{q q}^{(1)}\left(t, x, \vec{k}_{\perp}, \mu\right)= & \frac{\alpha_{s}(\mu) C_{F}}{2 \pi^{2}}\left\{\frac{2}{\mu^{2}} \mathcal{L}_{1}\left(\frac{t}{\mu^{2}}\right) \delta(1-x) \delta\left(\vec{k}_{\perp}^{2}\right)\right. \\
& +\frac{1}{\mu^{2}} \mathcal{L}_{0}\left(\frac{t}{\mu^{2}}\right)\left(1+x^{2}\right) \mathcal{L}_{0}(1-x) \delta\left(\vec{k}_{\perp}^{2}-\frac{(1-x) t}{x}\right) \\
& \left.+\delta(t) \delta\left(\vec{k}_{\perp}^{2}\right)\left[\left(1+x^{2}\right) \mathcal{L}_{1}(1-x)-\frac{\pi^{2}}{6} \delta(1-x)-\frac{1+x^{2}}{1-x} \ln x+1-x\right]\right\}, \\
\mathcal{I}_{q g}^{(1)}\left(t, x, \vec{k}_{\perp}, \mu\right)= & \frac{\alpha_{s}(\mu) T_{F}}{2 \pi^{2}}\left\{\left[\frac{1}{\mu^{2}} \mathcal{L}_{0}\left(\frac{t}{\mu^{2}}\right) \delta\left(\vec{k}_{\perp}^{2}-\frac{(1-x) t}{x}\right)+\delta(t) \delta\left(\vec{k}_{\perp}^{2}\right) \ln \frac{1-x}{x}\right]\right. \\
& \left.\times\left[x^{2}+(1-x)^{2}\right]+2 \delta(t) \delta\left(\vec{k}_{\perp}^{2}\right) x(1-x)\right\}
\end{aligned}
$$

where some additional factors of $1 / \pi$ are due to

$$
\delta^{2}\left(\vec{k}_{\perp}\right)=\frac{1}{\pi} \delta\left(\vec{k}_{\perp}^{2}\right) .
$$

The matching coefficients at NNLO have recently been calculated in ref. [85].

The TMD beam function satisfies a similar equation $[9,14,72,86]$

$$
B_{q}\left(x, \vec{k}_{\perp}, \mu, \nu\right)=\sum_{j} \int_{x}^{1} \frac{\mathrm{d} x^{\prime}}{x^{\prime}} \mathcal{I}_{q j}\left(\frac{x}{x^{\prime}}, \vec{k}_{\perp}, \mu, \nu\right) f_{j}\left(x^{\prime}, \mu\right)\left[1+\mathcal{O}\left(\frac{\Lambda_{\mathrm{QCD}}^{2}}{\vec{k}_{\perp}^{2}}\right)\right],
$$


with coefficients [87]

$\mathcal{I}_{q q}^{(0)}\left(x, \vec{k}_{\perp}, \mu, \nu\right)=\delta(1-x) \delta^{2}\left(\vec{k}_{\perp}\right)$,

$\mathcal{I}_{q g}^{(0)}\left(x, \vec{k}_{\perp}, \mu, \nu\right)=0$,

$\mathcal{I}_{q q}^{(1)}\left(x, \vec{k}_{\perp}, \mu, \nu\right)=\frac{\alpha_{s} C_{F}}{2 \pi^{2}}\left\{\frac{1}{\mu^{2}} \mathcal{L}_{0}\left(\frac{\vec{k}_{\perp}^{2}}{\mu^{2}}\right)\left[\left(1+x^{2}\right) \mathcal{L}_{0}(1-x)+2 \delta(1-x) \ln \frac{p^{-}}{\nu}\right]+\delta\left(\vec{k}_{\perp}^{2}\right)(1-x)\right\}$,

$\mathcal{I}_{q g}^{(1)}\left(x, \vec{k}_{\perp}, \mu, \nu\right)=\frac{\alpha_{s} T_{F}}{2 \pi^{2}}\left\{\frac{1}{\mu^{2}} \mathcal{L}_{0}\left(\frac{\vec{k}_{\perp}^{2}}{\mu^{2}}\right)\left[x^{2}+(1-x)^{2}\right]+2 \delta\left(\vec{k}_{\perp}^{2}\right) x(1-x)\right\}$.

Most approaches (such as in refs. [14, 17, 88]) do not (need to) separate the TMD beam and TMD soft function. In the SCET + regime, instead, we need the TMD beam function but have a different soft function.

\subsection{Soft functions}

The (beam) thrust soft function was determined at NLO in refs. [46, 89, 90]

$$
S\left(k^{+}, \mu\right)=\delta\left(k^{+}\right)+\frac{\alpha_{s} C_{F}}{2 \pi}\left[-\frac{8}{\mu} \mathcal{L}_{1}\left(\frac{k^{+}}{\mu}\right)+\frac{\pi^{2}}{6} \delta\left(k^{+}\right)\right]+\mathcal{O}\left(\alpha_{s}^{2}\right) .
$$

The NNLO contribution is known as well [80, 81].

We now calculate the FU soft function, which is differential in both $k^{+}$and $\vec{k}_{\perp}$, with $k^{+} \sim\left|\vec{k}_{\perp}\right| \cdot^{7}$ Starting from the definition in eq. (2.19), the tree-level result is

$$
S^{(0)}\left(k^{+}, \vec{k}_{\perp}\right)=\delta\left(k^{+}\right) \delta^{2}\left(\vec{k}_{\perp}\right)
$$

\footnotetext{
${ }^{7}$ This differs from the FU soft function in ref. [56], because their $k^{+}$measurement is independent of the hemisphere the gluon goes into.
} 
Using the rapidity regulator of refs. [71, 72], at one-loop order we find

$$
\begin{aligned}
& S^{(1)}\left(k^{+}, \vec{k}_{\perp}\right)=\frac{4 g^{2} w^{2} C_{F}}{(2 \pi)^{3-2 \epsilon}}\left(\frac{e^{\gamma_{E}} \mu^{2}}{4 \pi}\right)^{\epsilon} \nu^{\eta} \int \mathrm{d}^{d} \ell \theta\left(\ell^{0}\right) \delta\left(\ell^{2}\right) \frac{\left|2 \ell^{3}\right|^{-\eta}}{\ell^{-} \ell^{+}} \\
& \times \delta^{2}\left(\vec{\ell}_{\perp}-\vec{k}_{\perp}\right) \delta\left(\ell^{+} \theta\left(\ell^{-}-\ell^{+}\right)+\ell^{-} \theta\left(\ell^{+}-\ell^{-}\right)-k^{+}\right) \\
& =\alpha_{s} w^{2} C_{F} \frac{2^{1-\eta} e^{\epsilon \gamma_{E}} \mu^{2 \epsilon} \nu^{\eta}}{\pi^{2-\epsilon}} \int \mathrm{d}^{-2 \epsilon} \ell_{\epsilon} \frac{1}{\vec{k}_{\perp}^{2}+\vec{\ell}_{\epsilon}^{2}} \\
& \times \int_{0}^{\infty} \mathrm{d} \ell^{3} \frac{\left(\ell^{3}\right)^{-\eta}}{\sqrt{\vec{k}_{\perp}^{2}+\vec{\ell}_{\epsilon}^{2}+\left(\ell^{3}\right)^{2}}} \delta\left(\sqrt{\vec{k}_{\perp}^{2}+\vec{\ell}_{\epsilon}^{2}+\left(\ell^{3}\right)^{2}}-\ell^{3}-k^{+}\right) \\
& =\alpha_{s} w^{2} C_{F} \frac{2 e^{\epsilon \gamma_{E}} \mu^{2 \epsilon} \nu^{\eta}}{\pi^{2-\epsilon}} \frac{1}{\left(k^{+}\right)^{1-\eta}} \int \mathrm{d}^{-2 \epsilon} \ell_{\epsilon} \frac{\theta\left(\vec{k}_{\perp}^{2}-\left(k^{+}\right)^{2}+\vec{\ell}_{\epsilon}^{2}\right)}{\left(\vec{k}_{\perp}^{2}+\vec{\ell}_{\epsilon}^{2}\right)\left[\vec{k}_{\perp}^{2}-\left(k^{+}\right)^{2}+\vec{\ell}_{\epsilon}^{2}\right]^{\eta}} \\
& =\alpha_{s} w^{2} C_{F} \frac{2 e^{\epsilon \gamma_{E}} \mu^{2 \epsilon} \nu^{\eta}}{\pi^{2} \Gamma(-\epsilon)} \frac{1}{\left(k^{+}\right)^{1-\eta}\left(\vec{k}_{\perp}^{2}\right)^{1+\epsilon+\eta}} \int_{0}^{\infty} \mathrm{d} x \frac{\theta\left(x+1-\left(k^{+}\right)^{2} / \vec{k}_{\perp}^{2}\right)}{x^{1+\epsilon}(x+1)\left[x+1-\left(k^{+}\right)^{2} / \vec{k}_{\perp}^{2}\right]^{\eta}} \\
& =\alpha_{s} w^{2} C_{F} \frac{2 e^{\epsilon \gamma_{E}} \mu^{2 \epsilon+\eta} \nu^{\eta}}{\pi^{2} \Gamma(-\epsilon)}\left\{\frac{1}{\eta} \delta\left(k^{+}\right) \frac{1}{\left(\vec{k}_{\perp}^{2}\right)^{1+\epsilon+\eta}} \int_{0}^{\infty} \mathrm{d} x \frac{1}{x^{1+\epsilon}(x+1)^{1+\eta}}\right. \\
& +\frac{1}{\mu} \mathcal{L}_{0}\left(\frac{k^{+}}{\mu}\right) \frac{1}{\left(\vec{k}_{\perp}^{2}\right)^{1+\epsilon}}\left[\theta\left(\vec{k}_{\perp}^{2}-\left(k^{+}\right)^{2}\right) \int_{0}^{\infty} \mathrm{d} x \frac{1}{x^{1+\epsilon}(x+1)}\right. \\
& \left.\left.+\theta\left(\left(k^{+}\right)^{2}-\vec{k}_{\perp}^{2}\right) \int_{\left(k^{+}\right)^{2} / \vec{k}_{\perp}^{2}-1}^{\infty} \mathrm{d} x \frac{1}{x(x+1)}\right]+\mathcal{O}(\eta, \epsilon)\right\} \\
& \stackrel{\vec{k}_{\perp}^{2}>0}{=} \frac{\alpha_{s} w^{2} C_{F}}{\pi^{2}}\left\{\frac{2}{\eta}\left[-\frac{1}{\epsilon} \delta\left(\vec{k}_{\perp}^{2}\right)+\frac{1}{\mu^{2}} \mathcal{L}_{0}\left(\frac{\vec{k}_{\perp}^{2}}{\mu^{2}}\right)\right] \delta\left(k^{+}\right)+\frac{2}{\epsilon^{2}} \delta\left(\vec{k}_{\perp}^{2}\right) \delta\left(k^{+}\right)\right. \\
& +\frac{2}{\epsilon} \ln \frac{\mu}{\nu} \delta\left(\vec{k}_{\perp}^{2}\right) \delta\left(k^{+}\right)+2 \theta\left(\vec{k}_{\perp}^{2}-\left(k^{+}\right)^{2}\right) \frac{1}{\mu} \mathcal{L}_{0}\left(\frac{k^{+}}{\mu}\right) \frac{1}{\mu^{2}} \mathcal{L}_{0}\left(\frac{\vec{k}_{\perp}^{2}}{\mu^{2}}\right) \\
& \left.+\delta\left(k^{+}\right)\left[-\frac{2}{\mu^{2}} \mathcal{L}_{1}\left(\frac{\vec{k}_{\perp}^{2}}{\mu^{2}}\right)+\frac{2}{\mu^{2}} \mathcal{L}_{0}\left(\frac{\vec{k}_{\perp}^{2}}{\mu^{2}}\right) \ln \frac{\nu}{\mu}-\frac{\pi^{2}}{6} \delta\left(\vec{k}_{\perp}^{2}\right)\right]+\mathcal{O}(\eta, \epsilon)\right\} \\
& \stackrel{\vec{k}_{\perp}^{2} \geq 0}{\longrightarrow} \frac{\alpha_{s} w^{2} C_{F}}{\pi^{2}}\left\{\frac{2}{\eta}\left[-\frac{1}{\epsilon} \delta\left(\vec{k}_{\perp}^{2}\right)+\frac{1}{\mu^{2}} \mathcal{L}_{0}\left(\frac{\vec{k}_{\perp}^{2}}{\mu^{2}}\right)\right] \delta\left(k^{+}\right)+\frac{1}{\epsilon^{2}} \delta\left(\vec{k}_{\perp}^{2}\right) \delta\left(k^{+}\right)\right. \\
& +\frac{2}{\epsilon} \ln \frac{\mu}{\nu} \delta\left(\vec{k}_{\perp}^{2}\right) \delta\left(k^{+}\right)+\frac{2}{\mu^{3}} \mathcal{L}_{\Delta}\left(\frac{k^{+}}{\mu}, \frac{\vec{k}_{\perp}^{2}}{\mu^{2}}\right)+\delta\left(k^{+}\right)\left[-\frac{2}{\mu^{2}} \mathcal{L}_{1}\left(\frac{\vec{k}_{\perp}^{2}}{\mu^{2}}\right)\right. \\
& \left.\left.+\frac{2}{\mu^{2}} \mathcal{L}_{0}\left(\frac{\vec{k}_{\perp}^{2}}{\mu^{2}}\right) \ln \frac{\nu}{\mu}-\frac{\pi^{2}}{12} \delta\left(\vec{k}_{\perp}^{2}\right)\right]+\mathcal{O}(\eta, \epsilon)\right\} .
\end{aligned}
$$

Here longitudinal momenta get regulated by $\eta$, which can be thought of as the analog for rapidity divergences of the UV regulator $\epsilon$, with the dimensionful parameter $\nu$ acting like a renormalization scale. Both $1 / \eta$ and $1 / \epsilon$ divergences get absorbed in renormalization constants and give rise to $\mu$ - and $\nu$-RG equations. The bookkeeping parameter $w$ is used to derive the anomalous dimensions (see eq. (3.12)) and will be eventually set equal to 1.

In eq. (3.10) we introduce $x=\vec{\ell}_{\epsilon}^{2} / \vec{k}_{\perp}^{2}$ in intermediate steps, to simplify notation. In the second to last step, we first assume $\vec{k}_{\perp}^{2}>0$ to simplify the expansion in $\epsilon$. We then extend the distributions to include $\vec{k}_{\perp}^{2}=0$ and fix the coefficient of the $\delta\left(k^{+}\right) \delta\left(\vec{k}_{\perp}^{2}\right)$ by 
integrating the unexpanded result. The finite terms contain the following two-dimensional plus distribution

$$
\begin{aligned}
\mathcal{L}_{\Delta}\left(x_{1}, x_{2}\right)= & \lim _{\beta \rightarrow 0} \frac{\mathrm{d}}{\mathrm{d} x_{1}} \frac{\mathrm{d}}{\mathrm{d} x_{2}}\left[\theta\left(x_{2}-x_{1}^{2}\right) \theta\left(x_{1}-\beta\right) \ln x_{1}\left(\ln x_{2}-\ln x_{1}\right)\right. \\
& \left.+\frac{1}{4} \theta\left(x_{1}^{2}-x_{2}\right) \theta\left(x_{2}-\beta^{2}\right) \ln ^{2} x_{2}\right]
\end{aligned}
$$

The $1 / \epsilon$ and $1 / \eta$ poles are renormalized. We obtain the one-loop anomalous dimension in eq. (3.25) by using $[71,72]$

$$
\begin{aligned}
\frac{\mathrm{d} \alpha_{s}}{\mathrm{~d} \ln \mu} & =-2 \epsilon \alpha_{s}+\mathcal{O}\left(\alpha_{s}^{2}\right), \\
\frac{\mathrm{d} w}{\mathrm{~d} \ln \nu} & =-\frac{\eta}{2} w+\mathcal{O}\left(w^{2}\right),
\end{aligned}
$$

and setting $w=1$ afterwards. These are the same as for the TMD soft function. The renormalized FU soft function is given by the remaining finite terms,

$$
\begin{aligned}
S^{(1)}\left(k^{+}, \vec{k}_{\perp}, \mu, \nu\right)= & \frac{\alpha_{s} C_{F}}{\pi^{2}}\left\{\frac{2}{\mu^{3}} \mathcal{L}_{\Delta}\left(\frac{k^{+}}{\mu}, \frac{\vec{k}_{\perp}^{2}}{\mu^{2}}\right)\right. \\
& \left.+\delta\left(k^{+}\right)\left[-\frac{2}{\mu^{2}} \mathcal{L}_{1}\left(\frac{\vec{k}_{\perp}^{2}}{\mu^{2}}\right)+\frac{2}{\mu^{2}} \mathcal{L}_{0}\left(\frac{\vec{k}_{\perp}^{2}}{\mu^{2}}\right) \ln \frac{\nu}{\mu}-\frac{\pi^{2}}{12} \delta\left(\vec{k}_{\perp}^{2}\right)\right]\right\} .
\end{aligned}
$$

Its integral over $k^{+}$reproduces the TMD soft function in refs. [72, 87]

$$
\begin{aligned}
\int \mathrm{d} k^{+} S^{(1)}\left(k^{+}, \vec{k}_{\perp}, \mu, \nu\right) & =\frac{\alpha_{s} C_{F}}{\pi^{2}}\left[-\frac{1}{\mu^{2}} \mathcal{L}_{1}\left(\frac{\vec{k}_{\perp}^{2}}{\mu^{2}}\right)+\frac{1}{\mu^{2}} \mathcal{L}_{0}\left(\frac{\vec{k}_{\perp}^{2}}{\mu^{2}}\right) \ln \frac{\nu^{2}}{\mu^{2}}-\frac{\pi^{2}}{12} \delta\left(\vec{k}_{\perp}^{2}\right)\right] \\
& =S^{(1)}\left(\vec{k}_{\perp}, \mu, \nu\right)
\end{aligned}
$$

which parallels eq. (3.3) for the FU beam function. Here we used that for $x_{1}^{2}>x_{2}$,

$$
\int_{0}^{x_{1}} \mathrm{~d} x_{1}^{\prime} \mathcal{L}_{\Delta}\left(x_{1}^{\prime}, x_{2}\right)=\lim _{\beta \rightarrow 0} \frac{\mathrm{d}}{\mathrm{d} x_{2}}\left[\frac{1}{4} \theta\left(x_{2}-\beta^{2}\right) \ln ^{2} x_{2}\right]=\frac{1}{2} \mathcal{L}_{1}\left(x_{2}\right) .
$$

\subsection{Collinear-soft function}

The calculation of the collinear-soft function, defined in eq. (2.17), is actually quite similar to that of the FU soft function. The main difference is that collinear-soft radiation only goes into one hemisphere, leading to the change

$$
\delta\left(\ell^{+} \theta\left(\ell^{-}-\ell^{+}\right)+\ell^{-} \theta\left(\ell^{+}-\ell^{-}\right)-k^{+}\right) \rightarrow \delta\left(\ell^{+}-k^{+}\right) .
$$

We conveniently separate out a contribution $\frac{1}{2} S^{(1)}\left(k^{+}, \vec{k}_{\perp}\right)$ from the hemisphere where the measurement in the FU soft function and collinear-soft function are the same. The remain- 
der does not contain rapidity divergences, allowing us to set $\eta=0$ from the beginning,

$$
\begin{aligned}
& \mathscr{S}^{(1)}\left(k^{+}, \vec{k}_{\perp}\right)=\frac{4 g^{2} w^{2} C_{F}}{(2 \pi)^{3-2 \epsilon}}\left(\frac{e^{\gamma_{E}} \mu^{2}}{4 \pi}\right)^{\epsilon} \nu^{\eta} \int \mathrm{d}^{d} \ell \theta\left(\ell^{0}\right) \delta\left(\ell^{2}\right) \frac{\left|2 \ell^{3}\right|^{-\eta}}{\ell^{-} \ell^{+}} \delta^{2}\left(\vec{\ell}_{\perp}-\vec{k}_{\perp}\right) \delta\left(\ell^{+}-k^{+}\right) \\
& =\frac{1}{2} S^{(1)}+\alpha_{s} w^{2} C_{F} \frac{e^{\epsilon \gamma_{E}} \mu^{2 \epsilon}}{\pi^{2-\epsilon}} \int \mathrm{d}^{-2 \epsilon} \ell_{\epsilon} \frac{1}{\vec{k}_{\perp}^{2}+\vec{\ell}_{\epsilon}^{2}} \\
& \times \int_{0}^{\infty} \mathrm{d} \ell^{3} \frac{\delta\left(\sqrt{\vec{k}_{\perp}^{2}+\vec{\ell}_{\epsilon}^{2}+\left(\ell^{3}\right)^{2}}+\ell^{3}-k^{+}\right)}{\sqrt{\vec{k}_{\perp}^{2}+\vec{\ell}_{\epsilon}^{2}+\left(\ell^{3}\right)^{2}}} \\
& =\frac{1}{2} S^{(1)}+\alpha_{s} w^{2} C_{F} \frac{e^{\epsilon \gamma_{E}} \mu^{2 \epsilon}}{\pi^{2-\epsilon}} \frac{1}{k^{+}} \int \mathrm{d}^{-2 \epsilon} \ell_{\epsilon} \frac{\theta\left(\left(k^{+}\right)^{2}-\vec{k}_{\perp}^{2}-\vec{\ell}_{\epsilon}^{2}\right)}{\vec{k}_{\perp}^{2}+\vec{\ell}_{\epsilon}^{2}} \\
& =\frac{1}{2} S^{(1)}+\alpha_{s} w^{2} C_{F} \frac{e^{\epsilon \gamma_{E}} \mu^{2 \epsilon}}{\pi^{2} \Gamma(-\epsilon)} \frac{\theta\left(k^{+}-\left|\vec{k}_{\perp}\right|\right)}{k^{+}} \int_{0}^{\left(k^{+}\right)^{2}-\vec{k}_{\perp}^{2}} \mathrm{~d} \vec{\ell}_{\epsilon}^{2} \frac{1}{\left(\vec{\ell}_{\epsilon}^{2}\right)^{1+\epsilon}\left(\vec{k}_{\perp}^{2}+\vec{\ell}_{\epsilon}^{2}\right)} \\
& =\frac{1}{2} S^{(1)}+\alpha_{s} w^{2} C_{F} \frac{e^{\epsilon \gamma_{E}} \mu^{2 \epsilon}}{\pi^{2} \Gamma(1-\epsilon)} \frac{\theta\left(k^{+}-\left|\vec{k}_{\perp}\right|\right)}{\left[\left(k^{+}\right)^{2} / \vec{k}_{\perp}^{2}-1\right]^{\epsilon} k^{+}\left(\vec{k}_{\perp}^{2}\right)^{1+\epsilon}} \\
& \times{ }_{2} F_{1}\left(1,-\epsilon, 1-\epsilon, 1-\frac{\left(k^{+}\right)^{2}}{\vec{k}_{\perp}^{2}}\right) \\
& =\frac{1}{2} S^{(1)}+\frac{\alpha_{s} w^{2} C_{F}}{\pi^{2}}\left\{\frac{1}{2 \epsilon^{2}} \delta\left(k^{+}\right) \delta\left(\vec{k}_{\perp}^{2}\right)-\frac{1}{\epsilon} \frac{1}{\mu} \mathcal{L}_{0}\left(\frac{k^{+}}{\mu}\right) \delta\left(\vec{k}_{\perp}^{2}\right)+\frac{1}{\mu^{3}} \mathcal{L}_{\nabla}\left(\frac{k^{+}}{\mu}, \frac{\vec{k}_{\perp}^{2}}{\mu^{2}}\right)\right. \\
& \left.+\delta\left(k^{+}-\left|\vec{k}_{\perp}\right|\right)\left[\frac{2}{\mu} \mathcal{L}_{1}\left(\frac{k^{+}}{\mu}\right)-\frac{1}{2} \frac{1}{\mu^{2}} \mathcal{L}_{1}\left(\frac{\vec{k}_{\perp}^{2}}{\mu^{2}}\right)\right]-\frac{\pi^{2}}{12} \delta\left(k^{+}\right) \delta\left(\vec{k}_{\perp}^{2}\right)+\mathcal{O}(\epsilon)\right\} \\
& =\frac{\alpha_{s} w^{2} C_{F}}{\pi^{2}}\left\{\frac{1}{\eta}\left[-\frac{1}{\epsilon} \delta\left(\vec{k}_{\perp}^{2}\right)+\frac{1}{\mu^{2}} \mathcal{L}_{0}\left(\frac{\vec{k}_{\perp}^{2}}{\mu^{2}}\right)\right] \delta\left(k^{+}\right)+\frac{1}{\epsilon^{2}} \delta\left(k^{+}\right) \delta\left(\vec{k}_{\perp}^{2}\right)\right. \\
& -\frac{1}{\epsilon} \frac{1}{\mu} \mathcal{L}_{0}\left(\frac{k^{+}}{\mu}\right) \delta\left(\vec{k}_{\perp}^{2}\right)+\frac{1}{\epsilon} \ln \frac{\mu}{\nu} \delta\left(\vec{k}_{\perp}^{2}\right) \delta\left(k^{+}\right)+\frac{1}{\mu} \mathcal{L}_{0}\left(\frac{k^{+}}{\mu}\right) \frac{1}{\mu^{2}} \mathcal{L}_{0}\left(\frac{\vec{k}_{\perp}^{2}}{\mu^{2}}\right) \\
& \left.+\delta\left(k^{+}\right)\left[-\frac{1}{\mu^{2}} \mathcal{L}_{1}\left(\frac{\vec{k}_{\perp}^{2}}{\mu^{2}}\right)+\frac{1}{\mu^{2}} \mathcal{L}_{0}\left(\frac{\vec{k}_{\perp}^{2}}{\mu^{2}}\right) \ln \frac{\nu}{\mu}-\frac{\pi^{2}}{12} \delta\left(\vec{k}_{\perp}^{2}\right)\right]+\mathcal{O}(\eta, \epsilon)\right\} .
\end{aligned}
$$

The expansion in $\epsilon$ is again subtle at $\left(k^{+}, \vec{k}_{\perp}^{2}\right)=(0,0)$. Similar to section 3.3 , we first expand assuming $k^{+}>0$ and then extend the plus distributions to $k^{+}=0$, fixing the coefficient of $\delta\left(k^{+}\right) \delta\left(\vec{k}_{\perp}^{2}\right)$ by integration. In an intermediate expression, the following twodimensional plus distribution arises

$$
\begin{aligned}
\mathcal{L}_{\nabla}\left(x_{1}, x_{2}\right)=\lim _{\beta \rightarrow 0} \frac{\mathrm{d}}{\mathrm{d} x_{1}} \frac{\mathrm{d}}{\mathrm{d} x_{2}}[ & \theta\left(x_{1}^{2}-x_{2}\right) \theta\left(x_{2}-\beta^{2}\right)\left(\ln x_{1}-\frac{1}{4} \ln x_{2}\right) \ln x_{2} \\
& \left.+\theta\left(x_{2}-x_{1}^{2}\right) \theta\left(x_{1}-\beta\right) \ln ^{2} x_{1}\right] .
\end{aligned}
$$

In the final expression this combines with $\mathcal{L}_{\Delta}$ in eq. (3.10) to give

$$
\mathcal{L}_{\Delta}\left(x_{1}, x_{2}\right)+\mathcal{L}_{\nabla}\left(x_{1}, x_{2}\right)=\mathcal{L}_{0}\left(x_{1}\right) \mathcal{L}_{0}\left(x_{2}\right) .
$$


The divergences in eq. (3.17) lead to the one-loop anomalous dimensions in eq. (3.26). This satisfies the relation among anomalous dimensions required by consistency of the factorization theorem in eq. (2.15) at this order. The finite terms give

$$
\begin{aligned}
\mathscr{S}^{(1)}\left(k^{+}, \vec{k}_{\perp}, \mu, \nu\right)= & \frac{\alpha_{s} C_{F}}{\pi^{2}}\left\{\frac{1}{\mu} \mathcal{L}_{0}\left(\frac{k^{+}}{\mu}\right) \frac{1}{\mu^{2}} \mathcal{L}_{0}\left(\frac{\vec{k}_{\perp}^{2}}{\mu^{2}}\right)\right. \\
& \left.+\delta\left(k^{+}\right)\left[-\frac{1}{\mu^{2}} \mathcal{L}_{1}\left(\frac{\vec{k}_{\perp}^{2}}{\mu^{2}}\right)+\frac{1}{\mu^{2}} \mathcal{L}_{0}\left(\frac{\vec{k}_{\perp}^{2}}{\mu^{2}}\right) \ln \frac{\nu}{\mu}-\frac{\pi^{2}}{12} \delta\left(\vec{k}_{\perp}^{2}\right)\right]\right\} .
\end{aligned}
$$

\subsection{Renormalization and anomalous dimensions}

In this section we write down the RG equations (RGEs) for all these ingredients, which are well-known except for the FU soft function and collinear-soft function. Their anomalous dimensions are constrained by consistency of the factorization theorems in section 2.2 and agree with the one-loop calculations in sections 3.3 and 3.4. For completeness we give the expressions for both the quark and gluon case, as indicated by an additional index $i=q, g$ in this section. The anomalous dimensions involve the cusp anomalous dimension $\Gamma_{\text {cusp }}^{i}$ and non-cusp anomalous dimensions $\gamma_{H}^{i}, \gamma_{J}^{i}, \gamma_{\nu}^{i}$, which are tabulated in appendix B.

The anomalous dimension of the Wilson coefficient $C$ is

$$
\begin{aligned}
\mu \frac{\mathrm{d}}{\mathrm{d} \mu} C\left(Q^{2}, \mu\right) & =\gamma_{H}\left(Q^{2}, \mu\right) C\left(Q^{2}, \mu\right), \\
\gamma_{H}\left(Q^{2}, \mu\right) & =\Gamma_{\text {cusp }}^{q}\left(\alpha_{s}\right) \ln \frac{-Q^{2}-\mathrm{i} 0}{\mu^{2}}+\gamma_{H}^{q}\left(\alpha_{s}\right),
\end{aligned}
$$

from which the evolution of the hard function $H\left(Q^{2}, \mu\right)=\left|C\left(Q^{2}, \mu\right)\right|^{2}$ directly follows.

The FU beam function satisfies the following $\mathrm{RGE}^{8}$

$$
\begin{aligned}
\mu \frac{\mathrm{d}}{\mathrm{d} \mu} B_{i}\left(t, x, \vec{k}_{\perp}, \mu\right) & =\int_{0}^{t} \mathrm{~d} t^{\prime} \gamma_{B}^{i}\left(t-t^{\prime}, \mu\right) B_{i}\left(t^{\prime}, x, \vec{k}_{\perp}, \mu\right), \\
\gamma_{B}^{i}(t, \mu) & =-2 \Gamma_{\text {cusp }}^{i}\left(\alpha_{s}\right) \frac{1}{\mu^{2}} \mathcal{L}_{0}\left(\frac{t}{\mu^{2}}\right)+\gamma_{J}^{i}\left(\alpha_{s}\right) \delta(t) .
\end{aligned}
$$

The TMD beam function also involves a $\nu$ evolution (rapidity resummation) ${ }^{9}$

$$
\begin{aligned}
\mu \frac{\mathrm{d}}{\mathrm{d} \mu} B_{i}\left(x, \vec{k}_{\perp}, \mu, \nu\right) & =\gamma_{B}^{i}\left(p^{-}, \mu, \nu\right) B_{i}\left(x, \vec{k}_{\perp}, \mu, \nu\right), \\
\nu \frac{\mathrm{d}}{\mathrm{d} \nu} B_{i}\left(x, \vec{k}_{\perp}, \mu, \nu\right) & =\int \mathrm{d}^{2} \vec{k}_{\perp}^{\prime} \gamma_{\nu}^{i}\left(\vec{k}_{\perp}-\vec{k}_{\perp}^{\prime}, \mu\right) B_{i}\left(x, \vec{k}_{\perp}^{\prime}, \mu, \nu\right), \\
\gamma_{B}^{i}\left(p^{-}, \mu, \nu\right) & =2 \Gamma_{\text {cusp }}^{i}\left(\alpha_{s}\right) \ln \frac{\nu}{p^{-}}+\gamma_{J}^{i}\left(\alpha_{s}\right), \\
\gamma_{\nu}^{i}\left(\vec{k}_{\perp}, \mu\right) & =-\Gamma_{\text {cusp }}^{i}\left(\alpha_{s}\right) \frac{1}{\pi} \frac{1}{\mu^{2}} \mathcal{L}_{0}\left(\frac{\vec{k}_{\perp}^{2}}{\mu^{2}}\right)+\gamma_{\nu}^{i}\left(\alpha_{s}\right) \delta^{2}\left(\vec{k}_{\perp}\right) .
\end{aligned}
$$

\footnotetext{
${ }^{8}$ The additional spin structure [56] for the gluon beam function does not mix under renormalization and satisfies the same RGE.

${ }^{9}$ Its non-cusp $\mu$-anomalous dimension has not yet been calculated at two loops and is not fixed by consistency. However, the remaining degeneracy is irrelevant, since the TMD beam function has the same $\mu$ scale as the collinear-soft function (in $\mathrm{SCET}_{+}$) or FU soft function (in $\mathrm{SCET}_{\mathrm{II}}$ ).
} 
The RGE of the (beam) thrust soft function is given by

$$
\begin{aligned}
\mu \frac{\mathrm{d}}{\mathrm{d} \mu} S_{i}\left(k^{+}, \mu\right) & =\int_{0}^{k^{+}} \mathrm{d}{k^{\prime}}^{+} \gamma_{S}^{i}\left(k^{+}-{k^{\prime}}^{+}, \mu\right) S_{i}\left({k^{\prime}}^{+}, \mu\right), \\
\gamma_{S}^{i}\left(k^{+}, \mu\right) & =4 \Gamma_{\text {cusp }}^{i}\left(\alpha_{s}\right) \frac{1}{\mu} \mathcal{L}_{0}\left(\frac{k^{+}}{\mu}\right)-2\left[\gamma_{H}^{i}\left(\alpha_{s}\right)+\gamma_{J}^{i}\left(\alpha_{s}\right)\right] \delta\left(k^{+}\right),
\end{aligned}
$$

and for the FU soft function it is given by,

$$
\begin{aligned}
\mu \frac{\mathrm{d}}{\mathrm{d} \mu} S_{i}\left(k^{+}, \vec{k}_{\perp}, \mu, \nu\right) & =\gamma_{S}^{i}(\mu, \nu) S_{i}\left(k^{+}, \vec{k}_{\perp}, \mu, \nu\right), \\
\nu \frac{\mathrm{d}}{\mathrm{d} \nu} S_{i}\left(k^{+}, \vec{k}_{\perp}, \mu, \nu\right) & =-2 \int \mathrm{d}^{2} \vec{k}_{\perp}^{\prime} \gamma_{\nu}^{i}\left(\vec{k}_{\perp}-\vec{k}_{\perp}^{\prime}, \mu\right) S_{i}\left(k^{+}, \vec{k}_{\perp}^{\prime}, \mu, \nu\right), \\
\gamma_{S}^{i}(\mu, \nu) & =4 \Gamma_{\text {cusp }}^{i}\left(\alpha_{s}\right) \ln \frac{\mu}{\nu}-2\left[\gamma_{H}^{i}\left(\alpha_{s}\right)+\gamma_{J}^{i}\left(\alpha_{s}\right)\right],
\end{aligned}
$$

with $\gamma_{\nu}^{i}$ given in eq. (3.23).

The anomalous dimensions of the $n$-collinear-soft function and $\bar{n}$-collinear-soft function are identical. Using the $\mu$ and $\nu$ independence of the cross section in eq. (2.15), they are constrained by consistency to be

$$
\begin{aligned}
\mu \frac{\mathrm{d}}{\mathrm{d} \mu} \mathscr{S}_{i}\left(k^{+}, \vec{k}_{\perp}, \mu, \nu\right) & =\int_{0}^{k^{+}} \mathrm{d}{k^{\prime}}^{+} \gamma_{\mathscr{S}}^{i}\left(k^{+}-{k^{\prime}}^{+}, \mu, \nu\right) \mathscr{S}_{i}\left(k^{\prime+}, \vec{k}_{\perp}, \mu, \nu\right), \\
\nu \frac{\mathrm{d}}{\mathrm{d} \nu} \mathscr{S}_{i}\left(k^{+}, \vec{k}_{\perp}, \mu, \nu\right) & =-\int \mathrm{d}^{2} \vec{k}_{\perp}^{\prime} \gamma_{\nu}^{i}\left(\vec{k}_{\perp}-\vec{k}_{\perp}^{\prime}, \mu\right) \mathscr{S}_{i}\left(k^{+}, \vec{k}_{\perp}^{\prime}, \mu, \nu\right) \\
\gamma_{\mathscr{S}}^{i}\left(k^{+}, \mu, \nu\right) & =-2 \Gamma_{\text {cusp }}^{i}\left(\alpha_{s}\right)\left[\frac{1}{\mu} \mathcal{L}_{0}\left(\frac{k^{+}}{\mu}\right)+\ln \frac{\nu}{\mu} \delta\left(k^{+}\right)\right] .
\end{aligned}
$$

\subsection{NLL cross section}

At NLL, the cross section is generated by evolving the tree-level functions from their natural scale ${ }^{10}$

$$
\begin{aligned}
\mu_{H} & =-\mathrm{i} Q, \\
\mu_{B} & =p_{T}, \quad \nu_{B}=Q, \\
\mu_{\mathscr{S}} & =p_{T}, \quad \nu_{\mathscr{S}}=p_{T}^{2} / \mathcal{T}, \\
\mu_{S} & =\mathcal{T} .
\end{aligned}
$$

to a common scale using the RG equations in section 3.5. Evolving all functions to the collinear-soft scale $\left(\mu_{\mathscr{S}}, \nu_{\mathscr{S}}\right)$, using results from refs. [72, 90, 95-99], we obtain

$$
\begin{aligned}
& \int_{0}^{p_{T}} \mathrm{~d} p_{T}^{\prime} \int_{0}^{\mathcal{T}} \mathrm{d} \mathcal{T}^{\prime} \frac{\mathrm{d} \sigma}{\mathrm{d} Q^{2} \mathrm{~d} Y \mathrm{~d} p_{T}^{\prime} \mathrm{d} \mathcal{T}^{\prime}} \\
& =\sum_{q} \hat{\sigma}_{q}^{(0)}\left[f_{q}\left(x_{1}, \mu_{B}\right) f_{\bar{q}}\left(x_{2}, \mu_{B}\right)+f_{\bar{q}}\left(x_{1}, \mu_{B}\right) f_{q}\left(x_{2}, \mu_{B}\right)\right] \\
& \quad \times \frac{\Gamma\left(1-\eta_{B}\right) e^{\operatorname{Re}\left(K_{H}\right)+K_{B}+K_{S}-2 \gamma_{E} \eta_{B}-\gamma_{E}} \eta_{S}}{\Gamma\left(1+\eta_{B}\right) \Gamma\left(1+\eta_{S}\right)}\left|\left(\frac{-Q^{2}-\mathrm{i} 0}{\mu_{H}^{2}}\right)^{\eta_{H}}\right|\left(\frac{p_{T}}{\mu_{B}}\right)^{2 \eta_{B}}\left(\frac{\mathcal{T}}{\mu_{S}}\right)^{\eta_{S}},
\end{aligned}
$$

\footnotetext{
${ }^{10}$ The inclusion of the factor of $-\mathrm{i}$ in the hard scale $\mu_{H}$ follows from eq. (3.21) and allows us to resum a series of $\pi^{2}$-terms [91-94], thereby improving convergence.
} 
where $x_{1,2}=\left(Q / E_{\mathrm{cm}}\right) e^{ \pm Y}$ and $\operatorname{Re}(\ldots)$ denotes the real part of a complex number. The evolution kernels are given by

$$
\begin{array}{rlrl}
K_{H}\left(\mu_{H}, \mu_{\mathscr{S}}\right) & =-4 K_{\Gamma}^{q}\left(\mu_{H}, \mu_{\mathscr{S}}\right)+2 K_{\gamma_{H}^{q}}\left(\mu_{H}, \mu_{\mathscr{S}}\right), & & \eta_{H}\left(\mu_{B}, \mu_{\mathscr{S}}\right)=2 \eta_{\Gamma}^{q}\left(\mu_{B}, \mu_{\mathscr{S}}\right), \\
K_{B}\left(\nu_{B}, \nu_{\mathscr{S}}\right)=2 \gamma_{\nu}^{q}\left(\alpha_{S}\right) \ln \left(\frac{\nu_{\mathscr{S}}}{\nu_{B}}\right), & \eta_{B}\left(\nu_{B}, \nu_{\mathscr{S}}\right) & =-2 \eta_{\Gamma}^{q}\left(\nu_{B}, \nu_{\mathscr{S}}\right), \\
K_{S}\left(\mu_{S}, \mu_{\mathscr{S}}\right) & =-4 K_{\Gamma}^{q}\left(\mu_{S}, \mu_{\mathscr{S}}\right)+K_{\gamma_{S}^{q}}\left(\mu_{S}, \mu_{\mathscr{S}}\right), & & \eta_{S}\left(\mu_{S}, \mu_{\mathscr{S}}\right)=4 \eta_{\Gamma}^{q}\left(\mu_{S}, \mu_{\mathscr{S}}\right),
\end{array}
$$

in terms of functions given in appendix B. Since $\mu_{B}=\mu_{\mathscr{S}}$ there is no $\mu$-evolution for the beam functions. Because the scale of $\alpha_{s}$ in the $\nu$-evolution is $\mu$, the evolution of the non-cusp $\nu$-anomalous dimension takes the simpler form shown in $K_{B}$.

It is worth emphasizing that eq. (3.28) continuously merges with the $\mathrm{SCET}_{\mathrm{I}}$ and SCET $_{\text {II }}$ boundaries. This is no longer automatically achieved at NNLL, but can still be arranged, as discussed in the next section. We also stress that eq. (3.27) represents a naive choice of scales as these do no smoothly turn off at the boundaries leading to a discontinuity in the derivative of the cross section (see also the discussion around eq. (6.14)). This will be remedied by using profile functions [97, 100] in ref. [101], where a full analysis at NNLL will be presented.

\section{Matching the effective theories}

We now show that the continuous description of the cross section across the $\mathrm{SCET}_{\mathrm{I}}, \mathrm{SCET}_{+}$ and $\mathrm{SCET}_{\mathrm{II}}$ regions discussed in section 3.6 can naturally be extended to all orders. Specifically, in the $\mathrm{SCET}_{+}$region of phase space,

$$
\begin{aligned}
\mathcal{I}_{i j}\left(t, x, \vec{k}_{\perp}, \mu\right)= & \int \mathrm{d}^{2} \vec{k}_{\perp}^{\prime} \mathcal{I}_{i j}\left(x, \vec{k}_{\perp}^{\prime}, \mu, \nu\right) \mathscr{S}\left(t / p^{-}, \vec{k}_{\perp}-\vec{k}_{\perp}^{\prime}, \mu, \nu\right), \\
S\left(k^{+}, \vec{k}_{\perp}, \mu, \nu\right)= & \int \mathrm{d}^{2} \vec{k}_{\perp}^{\prime} \int \mathrm{d} k^{\prime+} \mathrm{d} k^{\prime \prime+} \mathscr{S}\left(k^{\prime+}, \vec{k}_{\perp}^{\prime}, \mu, \nu\right) \mathscr{S}\left(k^{\prime \prime}, \vec{k}_{\perp}-\vec{k}_{\perp}^{\prime}, \mu, \nu\right) \\
& \times S\left(k^{+}-k^{\prime+}-k^{\prime \prime+}, \mu\right),
\end{aligned}
$$

up to power corrections of $\mathcal{O}\left(\vec{k}_{\perp}^{2} / t\right)$ and $\mathcal{O}\left(\left(k^{+}\right)^{2} / \vec{k}_{\perp}^{2}\right)$, respectively. This follows directly from the consistency of the factorization theorems in section 2.2: when the resummation is turned off, i.e. a common renormalization scale is chosen for all functions in the factorization theorem, the $\mathrm{SCET}_{\mathrm{I}}$ and $\mathrm{SCET}_{\mathrm{II}}$ factorization theorems simply produce the full fixedorder cross section up to power corrections. As the $\mathrm{SCET}_{+}$regime involves an additional expansion, its fixed-order cross section can be obtained from either. Due to the many common ingredients between the $\mathrm{SCET}_{+}, \mathrm{SCET}_{\mathrm{I}}$ and $\mathrm{SCET}_{\mathrm{II}}$ factorization theorems, this then implies eq. (4.1).

We now restrict our attention to NNLL, for which eq. (4.1) reduces to

$$
\begin{aligned}
\mathcal{I}_{q q}^{(1)}\left(t, x, \vec{k}_{\perp}, \mu\right) & =\delta(t) \mathcal{I}_{q q}^{(1)}\left(x, \vec{k}_{\perp}, \mu, \nu\right)+\delta(1-x) \mathscr{S}^{(1)}\left(t / p^{-}, \vec{k}_{\perp}, \mu, \nu\right) \\
\mathcal{I}_{q g}^{(1)}\left(t, x, \vec{k}_{\perp}, \mu\right) & =\delta(t) \mathcal{I}_{q g}^{(1)}\left(x, \vec{k}_{\perp}, \mu\right), \\
S^{(1)}\left(k^{+}, \vec{k}_{\perp}, \mu, \nu\right) & =\frac{1}{\pi} \delta\left(\vec{k}_{\perp}^{2}\right) S^{(1)}\left(k^{+}, \mu\right)+2 \mathscr{S}^{(1)}\left(k^{+}, \vec{k}_{\perp}, \mu, \nu\right) .
\end{aligned}
$$


The first equations are valid up to corrections of $\mathcal{O}\left(\vec{k}_{\perp}^{2} / t\right)$, whereas the last one holds exactly for $k^{+}<\left|\vec{k}_{\perp}\right|$. This naturally suggests the following procedure for patching together the cross section at NNLL, 11

$$
\begin{aligned}
\frac{\mathrm{d}^{4} \sigma}{\mathrm{d} Q^{2} \mathrm{~d} Y \mathrm{~d} p_{T}^{2} \mathrm{~d} \mathcal{T}}= & \sum_{q} \hat{\sigma}_{q}^{0} H\left(Q^{2}, \mu\right) \int \mathrm{d} t_{1} \mathrm{~d} t_{2} \int \mathrm{d}^{2} \vec{k}_{1 \perp} \mathrm{d}^{2} \vec{k}_{2 \perp} \mathrm{d}^{2} \vec{k}_{1 \perp}^{\mathrm{cs}} \mathrm{d}^{2} \vec{k}_{2 \perp}^{\mathrm{cs}} \mathrm{d}^{2} \vec{k}_{\perp} \int \mathrm{d} k_{1}^{+} \mathrm{d} k_{2}^{+} \mathrm{d} k^{+} \\
& \times\left[B_{q}\left(t_{1}, x_{1}, \vec{k}_{1 \perp}, \mu\right)-\mathscr{S}^{(1)}\left(t_{1} e^{-Y} / Q, \vec{k}_{1 \perp}, \mu, \nu\right)\right] \mathscr{S}\left(k_{1}^{+}, \vec{k}_{1 \perp}^{\mathrm{cs}}, \mu, \nu\right) \\
& \times\left[B_{\bar{q}}\left(t_{2}, x_{2}, \vec{k}_{2 \perp}, \mu\right)-\mathscr{S}^{(1)}\left(t_{2} e^{Y} / Q, \vec{k}_{2 \perp}, \mu, \nu\right)\right] \mathscr{S}\left(k_{2}^{+}, \vec{k}_{2 \perp}^{\mathrm{cs}}, \mu, \nu\right) \\
& \times\left[S\left(k^{+}, \vec{k}_{\perp}, \mu, \nu\right)-2 \mathscr{S}^{(1)}\left(k^{+}, \vec{k}_{\perp}, \mu, \nu\right)\right] \delta\left(\mathcal{T}-\frac{e^{-Y} t_{1}+e^{Y} t_{2}}{Q}-k_{1}^{+}-k_{2}^{+}-k^{+}\right) \\
& \times \delta\left(p_{T}^{2}-\left|\vec{k}_{1 \perp}+\vec{k}_{2 \perp}+\vec{k}_{1 \perp}^{\mathrm{cs}}+\vec{k}_{2 \perp}^{\mathrm{cs}}+\vec{k}_{\perp}\right|^{2}\right)+(q \leftrightarrow \bar{q}) .
\end{aligned}
$$

Here the $\mathscr{S}^{(1)}$-term subtracted from the beam functions (soft function) are evaluated at the beam (soft) scale. From eq. (4.2) it follows that this reproduces the $\mathrm{SCET}_{\mathrm{I}}, \mathrm{SCET}_{+}$ and SCET $_{\text {II }}$ factorization theorems in eqs. (2.9), (2.15) and (2.18), up to power corrections.

We now derive eq. (4.2), using cumulants to avoid subtleties related to distributions. Starting with the boundary between $\mathrm{SCET}_{\mathrm{I}}$ and $\mathrm{SCET}_{+}$,

$$
\begin{aligned}
\int_{0}^{t} \mathrm{~d} t^{\prime} \int_{0}^{\vec{k}_{\perp}^{2}} \mathrm{~d} \vec{k}_{\perp}^{2} \mathcal{I}_{q g}^{(1)}\left(t^{\prime}, x, \vec{k}_{\perp}^{\prime}, \mu\right) & =\frac{\alpha_{s} T_{F}}{2 \pi^{2}}\left[\ln \min \left\{\frac{(1-x) t}{x \mu^{2}}, \frac{\vec{k}_{\perp}^{2}}{\mu^{2}}\right\} P_{q g}(x)+2 x(1-x)\right] \\
\int_{0}^{\vec{k}_{\perp}^{2}} \mathrm{~d} \vec{k}_{\perp}^{\prime} \mathcal{I}_{q g}^{(1)}\left(x, \vec{k}_{\perp}^{\prime}, \mu, \nu\right) & =\frac{\alpha_{s} T_{F}}{2 \pi^{2}}\left[\ln \left(\frac{\vec{k}_{\perp}^{2}}{\mu^{2}}\right) P_{q g}(x)+2 x(1-x)\right]
\end{aligned}
$$

We thus obtain the second line in eq. (4.2) for $0<x<1-\delta$, where

$$
\delta=\frac{\vec{k}_{\perp}^{2}}{t+\vec{k}_{\perp}^{2}}
$$

In the $\mathrm{SCET}_{+}$region of phase space, the size $\delta$ of the remaining interval $1-\delta \leq x \leq 1$ is parametrically small, implying that the contribution from this region to the cross section is power suppressed.

Similarly, we find that for $0<x<1-\delta$ the first line of eq. (4.2) is satisfied,

$$
\begin{aligned}
\int_{0}^{t} \mathrm{~d} t^{\prime} \int_{0}^{\vec{k}_{\perp}^{2}} \mathrm{~d} \vec{k}_{\perp}^{\prime 2} \mathcal{I}_{q q}^{(1)}\left(t^{\prime}, x, \vec{k}_{\perp}^{\prime}, \mu\right) & =\frac{\alpha_{s} C_{F}}{2 \pi^{2}}\left[\ln \left(\frac{\vec{k}_{\perp}^{2}}{\mu^{2}}\right) P_{q q}(x)+1-x\right] \\
& =\int_{0}^{\vec{k}_{\perp}^{2}} \mathrm{~d} \vec{k}_{\perp}^{\prime} \mathcal{I}_{q q}^{(1)}\left(x, \vec{k}_{\perp}^{\prime}, \mu, \nu\right) .
\end{aligned}
$$

\footnotetext{
${ }^{11}$ This has a natural generalization beyond NNLL in Fourier/Laplace space, where one can take the full inverse of $\mathscr{S}$ rather than the expanded version employed here.
} 
Although $1-\delta \leq x \leq 1$ is again parametrically small, the integral over this region is not, due to the presence of delta functions and plus distributions at $x=1$,

$$
\begin{aligned}
\int_{0}^{t} \mathrm{~d} t^{\prime} \int_{0}^{\vec{k}_{\perp}^{2}} \mathrm{~d} \vec{k}_{\perp}^{\prime} \int_{1-\delta}^{1} \mathrm{~d} x \mathcal{I}_{q q}^{(1)}\left(t^{\prime}, x, \vec{k}_{\perp}^{\prime}, \mu\right) & =\frac{\alpha_{s} C_{F}}{2 \pi^{2}}\left[\ln ^{2}\left(\frac{\delta t}{\mu^{2}}\right)-\frac{\pi^{2}}{6}+\mathcal{O}(\delta)\right], \\
\int_{0}^{\vec{k}_{\perp}^{2}} \mathrm{~d} \vec{k}_{\perp}^{\prime 2} \int_{1-\delta}^{1} \mathrm{~d} x \mathcal{I}_{q q}^{(1)}\left(x, \vec{k}_{\perp}^{\prime}, \mu\right) & =\frac{\alpha_{s} C_{F}}{2 \pi^{2}}\left[2 \ln \left(\frac{\vec{k}_{\perp}^{2}}{\mu^{2}}\right) \ln \left(\frac{\delta p^{-}}{\nu}\right)+\mathcal{O}(\delta)\right] .
\end{aligned}
$$

The mismatch is captured by the collinear-soft function

$$
\begin{aligned}
& \int_{0}^{t / p^{-}} \mathrm{d} k^{+} \int_{0}^{\vec{k}_{\perp}^{2}} \mathrm{~d} \vec{k}_{\perp}^{\prime} \mathscr{S}^{(1)}\left(k^{+}, \vec{k}_{\perp}^{\prime}, \mu, \nu\right) \\
& \quad=\frac{\alpha_{s} C_{F}}{2 \pi^{2}}\left[-\ln ^{2}\left(\frac{\vec{k}_{\perp}^{2}}{\mu^{2}}\right)+2 \ln \left(\frac{\vec{k}_{\perp}^{2}}{\mu^{2}}\right)\left(\ln \left(\frac{t}{\mu p^{-}}\right)+\ln \frac{\nu}{\mu}\right)-\frac{\pi^{2}}{6}\right]
\end{aligned}
$$

up to a power suppressed contribution

$$
\begin{aligned}
& \int_{0}^{\vec{k}_{\perp}^{2}} \mathrm{~d} \vec{k}_{\perp}^{\prime} \int_{1-\delta}^{1} \mathrm{~d} x\left[\int_{0}^{t} \mathrm{~d} t^{\prime} \mathcal{I}_{q q}^{(1)}\left(t^{\prime}, x, \vec{k}_{\perp}^{\prime}, \mu\right)-\mathcal{I}_{q q}^{(1)}\left(x, \vec{k}_{\perp}^{\prime}, \mu\right)\right] \\
& \quad-\int_{0}^{t / p^{-}} \mathrm{d} k^{+} \int_{0}^{\vec{k}_{\perp}^{2}} \mathrm{~d} \vec{k}_{\perp}^{\prime 2} \mathscr{S}^{(1)}\left(k^{+}, \vec{k}_{\perp}^{\prime}, \mu, \nu\right)=\frac{\alpha_{s} C_{F}}{2 \pi^{2}} \ln ^{2} \frac{\delta t}{\vec{k}_{\perp}^{2}}+\mathcal{O}(\delta)=\mathcal{O}(\delta) .
\end{aligned}
$$

Note that in the last line it important that $\delta$ is not arbitrary but given by eq. (4.5). Combined with eq. (4.6), this establishes the first line of eq. (4.2).

Lastly, we consider the boundary between $\mathrm{SCET}_{+}$and $\mathrm{SCET}_{\mathrm{II}}$, which involves the following ingredients

$$
\begin{aligned}
\int_{0}^{k^{+}} \mathrm{d} k^{\prime+} \int_{0}^{\vec{k}_{\perp}^{2}} \mathrm{~d} \vec{k}_{\perp}^{\prime 2} S^{(1)}\left(k^{\prime+}, \vec{k}_{\perp}^{\prime}, \mu, \nu\right)= & \frac{\alpha_{s} C_{F}}{2 \pi^{2}}\left[-2 \ln ^{2}\left(\frac{\vec{k}_{\perp}^{2}}{\mu^{2}}\right)+4 \ln \left(\frac{\vec{k}_{\perp}^{2}}{\mu^{2}}\right) \ln \left(\frac{k^{+}}{\mu}\right)-4 \ln ^{2}\left(\frac{k^{+}}{\mu}\right)\right. \\
& \left.+4 \ln \left(\frac{\vec{k}_{\perp}^{2}}{\mu^{2}}\right) \ln \frac{\nu}{\mu}-\frac{\pi^{2}}{6}+\theta\left(\left(k^{+}\right)^{2}-\vec{k}_{\perp}^{2}\right) \ln ^{2}\left(\frac{\vec{k}_{\perp}^{2}}{\left(k^{+}\right)^{2}}\right)\right], \\
\int_{0}^{k^{+}} \mathrm{d} k^{\prime+} S^{(1)}\left(k^{\prime+}, \mu\right)= & \frac{\alpha_{s} C_{F}}{2 \pi}\left[-4 \ln ^{2}\left(\frac{k^{+}}{\mu}\right)+\frac{\pi^{2}}{6}\right], \\
\int_{0}^{k^{+}} \mathrm{d} k^{\prime+} \int_{0}^{\vec{k}_{\perp}^{2}} \mathrm{~d} \vec{k}_{\perp}^{\prime 2} \mathscr{S}^{(1)}\left(k^{\prime+}, \vec{k}_{\perp}^{\prime}, \mu, \nu\right)= & \frac{\alpha_{s} C_{F}}{2 \pi^{2}}\left[-\ln ^{2}\left(\frac{\vec{k}_{\perp}^{2}}{\mu^{2}}\right)+2 \ln \left(\frac{\vec{k}_{\perp}^{2}}{\mu^{2}}\right)\left(\ln \left(\frac{k^{+}}{\mu}\right)+\ln \frac{\nu}{\mu}\right)-\frac{\pi^{2}}{6}\right] .
\end{aligned}
$$

It is straightforward to verify that for $k^{+}<k_{\perp}$ this satisfies the last line in eq. (4.2).

\section{$5 \quad$ NLO cross section}

In this section we determine the NLO cross section for $Z+0$ jet production, differential in the invariant mass $Q^{2}$, the rapidity $Y$ and $p_{T}$ of the $Z$ and beam thrust $\mathcal{T}$. We start by collecting the relevant ingredients in section 5.1, check the cancellation of IR divergences 
in section 5.2 and present the final result in section 5.3. In section 5.4 we verify that this agrees with $\mathrm{SCET}_{\mathrm{I}}, \mathrm{SCET}_{+}$and $\mathrm{SCET}_{\mathrm{II}}$, up to power corrections. This provides an important cross check of our formalism. We will match our resummed prediction onto these fixed-order corrections in ref. [101].

\section{$5.1 \quad$ Ingredients}

The partonic cross section for the one-loop real and virtual corrections in $\overline{\mathrm{MS}}$ are given by

$$
\begin{aligned}
& \hat{\sigma}_{q, R}^{(1)}=Q^{2} \hat{\sigma}_{q}^{(0)} 8 \pi \alpha_{s} C_{F}\left(\frac{e^{\gamma_{E}} \mu^{2}}{4 \pi}\right)^{\epsilon} \frac{1}{s_{q \bar{q} g}}\left[\frac{s_{q g}}{s_{\bar{q} g}}+\frac{s_{\bar{q} g}}{s_{q g}}+2 \frac{s_{q \bar{q}} s_{q \bar{q} g}}{s_{q g} s_{\bar{q} g}}-\epsilon\left(2+\frac{s_{q g}}{s_{\bar{q} g}}+\frac{s_{\bar{q} g}}{s_{q g}}\right)\right], \\
& \hat{\sigma}_{q, V}^{(1)}=Q^{2} \hat{\sigma}_{q}^{(0)} \frac{\alpha_{s} C_{F}}{\pi}\left(\frac{\mu^{2}}{s_{q \bar{q} g}}\right)^{\epsilon}\left[-\frac{1}{\epsilon^{2}}-\frac{3}{2 \epsilon}-4+\frac{7 \pi^{2}}{12}+\mathcal{O}(\epsilon)\right] .
\end{aligned}
$$

The Lorentz invariants that enter here are defined as

$$
s_{i j}=\left(p_{i}+p_{j}\right)^{2}=2 p_{i} \cdot p_{j}, \quad s_{i j k}=\left(p_{i}+p_{j}+p_{k}\right)^{2}=s_{i j}+s_{i k}+s_{j k},
$$

using an incoming momentum convention for $p_{i}$. Due to the flavor dependence of the treelevel partonic cross section $\hat{\sigma}_{q}^{(0)}$, we will for simplicity restrict ourselves to a single quark flavor. The full cross section can be obtained by summing over quark flavors.

We now discuss kinematics and phase space. The incoming partons have momenta

$$
p_{1}=\left(x_{1} E_{\mathrm{cm}}, 0,0\right), \quad p_{2}=\left(0, x_{2} E_{\mathrm{cm}}, 0\right)
$$

in $(-,+, \perp)$ light-cone coordinates (see eq. (1.2)), with $x_{1,2}$ the momentum fractions of the partons with respect to the colliding hadrons. At LO the final state consists of a $Z$ boson with momentum $q^{\mu}$, and the phase space integral yields

$$
\begin{aligned}
\int \mathrm{d} \Phi_{i j}^{(0)}= & \int \frac{\mathrm{d} x_{1}}{x_{1}} \frac{\mathrm{d} x_{2}}{x_{2}} f_{i}\left(x_{1}, \mu\right) f_{j}\left(x_{2}, \mu\right) \int \frac{\mathrm{d}^{d} q}{(2 \pi)^{d}}(2 \pi)^{d} \delta\left(q-p_{1}-p_{2}\right) \\
& \times \delta\left(Q^{2}-q^{2}\right) \delta\left[Y-\frac{1}{2} \ln \left(\frac{q^{-}}{q^{+}}\right)\right] \delta\left(p_{T}^{2}-\vec{q}_{\perp}^{2}\right) \delta(\mathcal{T}) \\
= & \frac{1}{Q^{2}} \delta\left(p_{T}^{2}\right) \delta(\mathcal{T}) f_{i}\left(\frac{Q}{E_{\mathrm{cm}}} e^{Y}, \mu\right) f_{j}\left(\frac{Q}{E_{\mathrm{cm}}} e^{-Y}, \mu\right)
\end{aligned}
$$

At this order, the momentum fractions $x_{1,2}$ and the momentum of the $Z$ are thus

$$
x_{1}=\frac{Q}{E_{\mathrm{cm}}} e^{Y}, \quad x_{2}=\frac{Q}{E_{\mathrm{cm}}} e^{-Y}, \quad q=\left(Q e^{Y}, Q e^{-Y}, 0\right) .
$$

At NLO, there is an additional massless parton that the $Z$-boson can recoil against. To be consistent with eq. (5.1), we use an incoming convention for the momentum $p_{3}$ of this parton. Assuming for simplicity that this parton goes into the right hemisphere, 
$-p_{3}^{+}<-p_{3}^{-}$, the phase space is given by

$$
\begin{aligned}
\int \mathrm{d} \Phi_{i j, \mathrm{R}}^{(1)}= & \int \frac{\mathrm{d} x_{1}}{x_{1}} \frac{\mathrm{d} x_{2}}{x_{2}} f_{i}\left(x_{1}, \mu\right) f_{j}\left(x_{2}, \mu\right) \int \frac{\mathrm{d}^{d} q}{(2 \pi)^{d}} \int \frac{\mathrm{d}^{d} p_{3}}{(2 \pi)^{d}} \theta\left(-p_{3}^{0}\right) 2 \pi \delta\left(p_{3}^{2}\right)(2 \pi)^{d} \delta\left(q-p_{1}-p_{2}-p_{3}\right) \\
& \times \delta\left(Q^{2}-q^{2}\right) \delta\left[Y-\frac{1}{2} \ln \left(\frac{q^{-}}{q^{+}}\right)\right] \delta\left(p_{T}^{2}-\vec{q}_{\perp}^{2}\right) \theta\left(p_{3}^{+}-p_{3}^{-}\right) \delta\left(\mathcal{T}+p_{3}^{+}\right) \\
= & \frac{1}{(4 \pi)^{2-\epsilon} \Gamma(1-\epsilon)} \frac{\theta\left(p_{T}-\mathcal{T}\right)}{p_{T}^{2 \epsilon}\left(\sqrt{Q^{2}+p_{T}^{2}} e^{Y} \mathcal{T}+p_{T}^{2}\right)\left(\sqrt{Q^{2}+p_{T}^{2}} e^{-Y}+\mathcal{T}\right)} \\
& \times f_{i}\left(\frac{\sqrt{Q^{2}+p_{T}^{2}} e^{Y}}{E_{\mathrm{cm}}}+\frac{p_{T}^{2}}{E_{\mathrm{cm}} \mathcal{T}}, \mu\right) f_{j}\left(\frac{\sqrt{Q^{2}+p_{T}^{2}} e^{-Y}}{E_{\mathrm{cm}}}+\frac{\mathcal{T}}{E_{\mathrm{cm}}}, \mu\right)
\end{aligned}
$$

The contribution for the other hemisphere $\mathrm{d} \Phi_{i j, \mathrm{~L}}^{(1)}$ can be obtained in a similar manner. From this we can read off

$$
\begin{array}{rlrl}
x_{1} & =\frac{\sqrt{Q^{2}+p_{T}^{2}} e^{Y}}{E_{\mathrm{cm}}}+\frac{p_{T}^{2}}{E_{\mathrm{cm}} \mathcal{T}}, & x_{2}=\frac{\sqrt{Q^{2}+p_{T}^{2}} e^{-Y}}{E_{\mathrm{cm}}}+\frac{\mathcal{T}}{E_{\mathrm{cm}}}, \\
q=\left(\sqrt{Q^{2}+p_{T}^{2}} e^{Y}, \sqrt{Q^{2}+p_{T}^{2}} e^{-Y}, p_{T}\right), & p_{3}=\left(\frac{-p_{T}^{2}}{\mathcal{T}},-\mathcal{T}, p_{T}\right) .
\end{array}
$$

The (irrelevant) azimuthal angle in the transverse plane is not fixed by the measurement. It is straightforward to evaluate the invariants in eq. (5.1) in terms of eq. (5.7). For $q \bar{q} \rightarrow Z g$,

$$
s_{q \bar{q}}=x_{1} x_{2} E_{\mathrm{cm}}^{2}, \quad s_{q g}=-x_{1} E_{\mathrm{cm}} \mathcal{T}, \quad s_{\bar{q} g}=-x_{2} E_{\mathrm{cm}} \frac{p_{T}^{2}}{\mathcal{T}} .
$$

The other cases can be obtained by permutations. For $g q \rightarrow Z q$ we have

$$
s_{q \bar{q}}=-x_{2} E_{\mathrm{cm}} \frac{p_{T}^{2}}{\mathcal{T}}, \quad s_{q g}=x_{1} x_{2} E_{\mathrm{cm}}^{2}, \quad s_{\bar{q} g}=-x_{1} E_{\mathrm{cm}} \mathcal{T}
$$

and for $q g \rightarrow Z q$ we have

$$
s_{q \bar{q}}=-x_{1} E_{\mathrm{cm}} \mathcal{T}, \quad s_{q g}=x_{1} x_{2} E_{\mathrm{cm}}^{2}, \quad s_{\bar{q} g}=-x_{2} E_{\mathrm{cm}} \frac{p_{T}^{2}}{\mathcal{T}} .
$$

Lastly, there is the NLO contribution from the PDFs, which consists of pure IR poles in dimensional regularization. This can be effectively described as

$$
f_{q}^{(1)}(x, \mu)=\frac{\alpha_{s}}{2 \pi} \frac{1}{\epsilon} \sum_{j} \int_{x}^{1} \frac{\mathrm{d} x^{\prime}}{x^{\prime}} C_{j} P_{q j}\left(\frac{x}{x^{\prime}}\right) f_{j}\left(x^{\prime}, \mu\right),
$$

where the color factor $C_{j}$ is $C_{F}\left(T_{F}\right)$ for $j=q(j=g)$ and the splitting functions are

$$
P_{q q}(z)=\left(1+z^{2}\right) \mathcal{L}_{0}(1-z)+\frac{3}{2} \delta(1-z), \quad P_{q g}(z)=(1-z)^{2}+z^{2} .
$$




\subsection{Cancellation of IR divergences}

In this section we combine these ingredients and verify the cancellation of IR divergences. We assume $p_{T}, \mathcal{T} \ll Q$ to simplify the calculation, though we do not restrict to any particular relative hierarchy between $p_{T}$ and $\mathcal{T}$. This leads to

$$
\begin{aligned}
& \hat{\sigma}_{q \bar{q} \rightarrow Z g}=Q^{2} \hat{\sigma}_{q}^{(0)} 8 \pi \alpha_{s} C_{F}\left(\frac{e^{\gamma_{E}} \mu^{2}}{4 \pi}\right)^{\epsilon}\left[\frac{2}{p_{T}^{2}}+(1-\epsilon) \frac{p_{T}^{2}}{x_{1} E_{\mathrm{cm}}\left(x_{1} E_{\mathrm{cm}} \mathcal{T}-p_{T}^{2}\right) \mathcal{T}}\right]\left[1+\mathcal{O}\left(\frac{\mathcal{T}}{Q}, \frac{p_{T}^{2}}{Q^{2}}\right)\right] \\
& \hat{\sigma}_{g q \rightarrow Z q}=Q^{2} \hat{\sigma}_{q}^{(0)} 8 \pi \alpha_{s} T_{F}\left(\frac{e^{\gamma_{E}} \mu^{2}}{4 \pi}\right)^{\epsilon}\left[\frac{1}{x_{1} E_{\mathrm{cm}} \mathcal{T}-p_{T}^{2}}-\frac{2}{1-\epsilon} \frac{p_{T}^{2}}{x_{1}^{2} E_{\mathrm{cm}}^{2} \mathcal{T}^{2}}\right]\left[1+\mathcal{O}\left(\frac{\mathcal{T}}{Q}, \frac{p_{T}^{2}}{Q^{2}}\right)\right] \\
& \hat{\sigma}_{q g \rightarrow Z q}=Q^{2} \hat{\sigma}_{q}^{(0)} 8 \pi \alpha_{s} T_{F}\left(\frac{e^{\gamma_{E}} \mu^{2}}{4 \pi}\right)^{\epsilon} \frac{x_{1} \mathcal{T}^{2}}{x_{2} p_{T}^{2}\left(x_{1} E_{\mathrm{cm}} \mathcal{T}-p_{T}^{2}\right)}\left[1+\mathcal{O}\left(\frac{\mathcal{T}}{Q}, \frac{p_{T}^{2}}{Q^{2}}\right)\right] .
\end{aligned}
$$

For $q g \rightarrow Z q$ and $g q \rightarrow Z q$ there is a fermion minus sign from crossing eq. (5.1) and we have taken into account that we need to average over incoming gluon polarizations and colors instead of quark spins and colors, resulting in the overall factor $2 N_{c} /\left[(2-2 \epsilon)\left(N_{c}^{2}-1\right)\right]$. The phase space in eq. (5.6) simplifies as well

$$
\int \mathrm{d} \Phi_{i j, R}^{(1)}=\frac{1}{(4 \pi)^{2-\epsilon} \Gamma(1-\epsilon)} \frac{\theta\left(p_{T}-\mathcal{T}\right)}{Q\left(Q \mathcal{T}+p_{T}^{2} e^{-Y}\right) p_{T}^{2 \epsilon}} f_{i}\left(\frac{Q e^{Y}}{E_{\mathrm{cm}}}+\frac{p_{T}^{2}}{E_{\mathrm{cm}} \mathcal{T}}, \mu\right) f_{j}\left(\frac{Q e^{-Y}}{E_{\mathrm{cm}}}, \mu\right) .
$$

To avoid subtleties related to distributions, we calculate the cumulative cross section in $p_{T}$ and $\mathcal{T}$,

$$
\begin{aligned}
& \int_{0}^{p_{T}^{2}} \mathrm{~d} p_{T}^{\prime 2} \int_{0}^{\mathcal{T}} \mathrm{d} \mathcal{T}^{\prime} \frac{\mathrm{d}^{4} \sigma_{q}^{(1)}}{\mathrm{d} Q^{2} \mathrm{~d} Y \mathrm{~d} p_{T}^{\prime 2} \mathrm{~d} \mathcal{T}^{\prime}} \\
& =\int_{0}^{p_{T}^{2}} \mathrm{~d} p_{T}^{\prime 2} \int_{0}^{\mathcal{T}} \mathrm{d} \mathcal{T}^{\prime}\left\{\int \mathrm{d} \Phi_{q \bar{q}}^{(0)}\left[\frac{1}{2} \hat{\sigma}_{q, V}^{(1)}+\hat{\sigma}_{q}^{(0)} \frac{f_{q}^{(1)}\left(x_{1}, \mu\right)}{f_{q}\left(x_{1}, \mu\right)}\right]+\int \mathrm{d} \Phi_{q \bar{q}, R}^{(1)} \hat{\sigma}_{q \bar{q} \rightarrow Z g}\right. \\
& \left.+\int \mathrm{d} \Phi_{q g, R}^{(1)} \hat{\sigma}_{q g \rightarrow Z q}+\int \mathrm{d} \Phi_{g q, R}^{(1)} \hat{\sigma}_{g q \rightarrow Z q}+\left(x_{1} \leftrightarrow x_{2}\right)+(q \leftrightarrow \bar{q})\right\} \\
& =\hat{\sigma}_{q}^{(0)} f_{q}\left(x_{1}, \mu\right) f_{\bar{q}}\left(x_{2}, \mu\right) \frac{\alpha_{s}}{2 \pi}\left(C_{F}\left(\frac{\mu^{2}}{Q^{2}}\right)^{\epsilon}\left[-\frac{1}{\epsilon^{2}}-\frac{3}{2 \epsilon}-4+\frac{7 \pi^{2}}{12}+\mathcal{O}(\epsilon)\right]\right. \\
& +\frac{1}{\epsilon} \sum_{j} \int_{x_{1}}^{1} \frac{\mathrm{d} x_{1}^{\prime}}{x_{1}^{\prime}} \frac{f_{j}\left(x_{1}^{\prime}, \mu\right)}{f_{q}\left(x_{1}, \mu\right)} C_{j} P_{q j}\left(\frac{x_{1}}{x_{1}^{\prime}}\right) \\
& -\frac{1}{\epsilon} \int_{x_{1}}^{1} \frac{\mathrm{d} x_{1}^{\prime}}{x_{1}^{\prime}}\left\{\frac{f_{q}\left(x_{1}^{\prime}, \mu\right)}{f_{q}\left(x_{1}, \mu\right)} C_{F}\left[\frac{2 x_{1}}{\left(x_{1}^{\prime}-x_{1}\right)^{1+2 \epsilon}}+(1-\epsilon) \frac{\left(x_{1}^{\prime}-x_{1}\right)^{1-2 \epsilon}}{x_{1}^{\prime}}\right]\right. \\
& \left.+\frac{f_{g}\left(x_{1}^{\prime}, \mu\right)}{f_{q}\left(x_{1}, \mu\right)} T_{F}\left[\frac{1}{\left(x_{1}^{\prime}-x_{1}\right)^{2 \epsilon}}-\frac{2}{1-\epsilon} \frac{x_{1}\left(x_{1}^{\prime}-x_{1}\right)^{1-2 \epsilon}}{x_{1}^{\prime 2}}\right]\right\} \frac{e^{\epsilon \gamma_{E}}}{\Gamma(1-\epsilon)}\left(\frac{\mu^{2}}{E_{\mathrm{cm}}^{2}}\right)^{\epsilon} \\
& \times\left[\min \left\{1, \frac{\mathcal{T}}{\left(x_{1}^{\prime}-x_{1}\right) E_{\mathrm{cm}}}, \frac{p_{T}^{2}}{\left(x_{1}^{\prime}-x_{1}\right)^{2} E_{\mathrm{cm}}^{2}}\right\}\right]^{-\epsilon} \\
& +\int_{x_{1}}^{1} \frac{\mathrm{d} x_{1}^{\prime}}{x_{1}^{\prime}} \frac{f_{q}\left(x_{1}^{\prime}, \mu\right) f_{g}\left(x_{2}, \mu\right)}{f_{q}\left(x_{1}, \mu\right) f_{\bar{q}}\left(x_{2}, \mu\right)} T_{F} \frac{x_{1}^{\prime}}{x_{2}\left(x_{1}^{\prime}-x_{1}\right)^{2 \epsilon}} \frac{e^{\epsilon \gamma_{E}}}{\Gamma(2-\epsilon)}\left(\frac{\mu^{2}}{E_{\mathrm{cm}}^{2}}\right)^{\epsilon} \\
& \left.\times\left[\min \left\{1, \frac{\mathcal{T}}{\left(x_{1}^{\prime}-x_{1}\right) E_{\mathrm{cm}}}, \frac{p_{T}^{2}}{\left(x_{1}^{\prime}-x_{1}\right)^{2} E_{\mathrm{cm}}^{2}}\right\}\right]^{1-\epsilon}\right)+\left(x_{1} \leftrightarrow x_{2}\right)+(q \leftrightarrow \bar{q}),
\end{aligned}
$$


where in the final expression we used the shorthand notation

$$
x_{1}=\frac{Q}{E_{\mathrm{cm}}} e^{Y}, \quad x_{2}=\frac{Q}{E_{\mathrm{cm}}} e^{-Y}
$$

which should not to be confused with eq. (5.7). The contribution from $\mathrm{d} \Phi_{L}$ is included through $\left(x_{1} \leftrightarrow x_{2}\right)$.

We obtained eq. (5.15) by first rewriting the $p_{T}^{\prime 2}$ integral in terms of $x^{\prime}$

$$
p_{T}^{\prime 2}=\left(x_{1}^{\prime}-x_{1}\right) E_{\mathrm{cm}} \mathcal{T}^{\prime}
$$

such that

$$
\int_{0}^{p_{T}^{2}} \mathrm{~d} p_{T}^{\prime 2}=\int_{x_{1}}^{1} \mathrm{~d} x_{1}^{\prime} E_{\mathrm{cm}} \mathcal{T}^{\prime} \theta\left(p_{T}^{2}-\left(x_{1}^{\prime}-x_{1}\right) E_{\mathrm{cm}} \mathcal{T}^{\prime}\right)
$$

For the subsequent $\mathcal{T}^{\prime}$ integral we find

$$
\begin{aligned}
& \int_{0}^{\mathcal{T}} \mathrm{d} \mathcal{T}^{\prime} \frac{\theta\left(p_{T}^{2}-\left(x_{1}^{\prime}-x_{1}\right) E_{\mathrm{cm}} \mathcal{T}^{\prime}\right) \theta\left(\left(x_{1}^{\prime}-x_{1}\right) E_{\mathrm{cm}}-\mathcal{T}^{\prime}\right)}{\left(\mathcal{T}^{\prime}\right)^{1+\epsilon}} \\
& \quad=-\frac{1}{\epsilon} \frac{\theta\left(x_{1}^{\prime}-x_{1}\right)}{\left(x_{1}^{\prime}-x_{1}\right)^{\epsilon} E_{\mathrm{cm}}^{\epsilon}}\left[\min \left\{1, \frac{\mathcal{T}}{\left(x_{1}^{\prime}-x_{1}\right) E_{\mathrm{cm}}}, \frac{p_{T}^{2}}{\left(x_{1}^{\prime}-x_{1}\right)^{2} E_{\mathrm{cm}}^{2}}\right\}\right]^{-\epsilon}
\end{aligned}
$$

and similarly for the term that goes like $\left(\mathcal{T}^{\prime}\right)^{-\epsilon}$. The cancellation of IR divergences becomes clear once we use the following expressions to expand in $\epsilon$,

$$
\begin{aligned}
& \frac{2 x_{1}}{\left(x_{1}^{\prime}-x_{1}\right)^{1+2 \epsilon}}+(1-\epsilon) \frac{\left(x_{1}^{\prime}-x_{1}\right)^{1-2 \epsilon}}{x_{1}^{\prime}}=\left(-\frac{1}{\epsilon}-\frac{3}{2}+2 \ln x_{1}\right) \delta\left(1-\frac{x_{1}}{x_{1}^{\prime}}\right)+P_{q q}\left(\frac{x_{1}}{x_{1}^{\prime}}\right)+\mathcal{O}(\epsilon), \\
& \frac{1}{\left(x_{1}^{\prime}-x_{1}\right)^{2 \epsilon}}-\frac{2}{1-\epsilon} \frac{x_{1}\left(x_{1}^{\prime}-x_{1}\right)^{1-2 \epsilon}}{x_{1}^{\prime 2}}=P_{q g}\left(\frac{x_{1}}{x_{1}^{\prime}}\right)+\mathcal{O}(\epsilon)
\end{aligned}
$$

which follow from eq. (A.3). Note that the $\ln x_{1}$ term on the first line and the corresponding term from $\left(x_{1} \leftrightarrow x_{2}\right)$ combine with $\ln \left(E^{2} / \mu^{2}\right)$ to give $\ln \left(Q^{2} / \mu^{2}\right)=\ln x_{1}+\ln x_{2}+\ln \left(E^{2} / \mu^{2}\right)$.

\section{$5.3 \quad$ Result}

We now present the cross section for $p p \rightarrow Z+0$ jets, differential in the invariant mass and rapidity of the $Z$, and with cuts on the transverse momentum of the $Z$ and on beam thrust. This is given by the finite $\mathcal{O}\left(\epsilon^{0}\right)$ terms in eq. (5.15), which we rearrange into the 
following form

$$
\begin{aligned}
& \int_{0}^{p_{T}^{2}} \mathrm{~d} p_{T}^{\prime 2} \int_{0}^{\mathcal{T}} \mathrm{d} \mathcal{T}^{\prime} \frac{\mathrm{d}^{4} \sigma_{q}^{(1)}}{\mathrm{d} Q^{2} \mathrm{~d} Y \mathrm{~d} p_{T}^{\prime 2} \mathrm{~d} \mathcal{T}^{\prime}} \\
& =\hat{\sigma}_{q}^{(0)} f_{q}\left(x_{1}, \mu\right) f_{\bar{q}}\left(x_{2}, \mu\right) \frac{\alpha_{s}}{2 \pi}\left(2\left[\ln ^{2}\left(\frac{x_{1} E_{\mathrm{cm}}}{\mu}\right)-\ln ^{2}\left(\frac{Q}{\mu}\right)\right]\right. \\
& \quad+\sum_{j} \int_{x_{1}}^{1} \frac{\mathrm{d} z_{1}}{z_{1}} \frac{f_{j}\left(x_{1} / z_{1}, \mu\right)}{f_{q}\left(x_{1}, \mu\right)} C_{j} P_{q j}\left(z_{1}\right) \ln \min \left\{\frac{x_{1}^{2} E_{\mathrm{cm}}^{2}}{z_{1}^{2} \mu^{2}}, \frac{\mathcal{T} x_{1} E_{\mathrm{cm}}}{z_{1}\left(1-z_{1}\right) \mu^{2}}, \frac{p_{T}^{2}}{\left(1-z_{1}\right)^{2} \mu^{2}}\right\} \\
& \quad+\int_{x_{1}}^{1} \frac{\mathrm{d} z_{1}}{z_{1}}\left\{\frac{f_{q}\left(x_{1} / z_{1}, \mu\right)}{f_{q}\left(x_{1}, \mu\right)} C_{F}\left[2\left(1+z_{1}^{2}\right) \mathcal{L}_{1}\left(1-z_{1}\right)+\left(-4+\frac{\pi^{2}}{2}\right) \delta\left(1-z_{1}\right)+1-z_{1}\right]\right. \\
& \left.\quad+\frac{f_{g}\left(x_{1} / z_{1}, \mu\right)}{f_{q}\left(x_{1}, \mu\right)} T_{F}\left[2 P_{q g}\left(z_{1}\right) \ln \left(1-z_{1}\right)+2 z_{1}\left(1-z_{1}\right)\right]\right\} \\
& \left.\quad+\int_{x_{1}}^{1} \frac{\mathrm{d} z_{1}}{z_{1}} \frac{f_{q}\left(x_{1} / z_{1}, \mu\right) f_{g}\left(x_{2}, \mu\right)}{f_{q}\left(x_{1}, \mu\right) f_{\bar{q}}\left(x_{2}, \mu\right)} T_{F} \frac{x_{1}}{z_{1} x_{2}} \min \left\{1, \frac{z_{1} p_{T}^{2}}{x_{1}\left(1-z_{1}\right) E_{\mathrm{cm}}}, \frac{z_{1}^{2}\left(1-z_{1}\right)^{2} E_{\mathrm{cm}}^{2}}{x_{1}}\right\}\right) \\
& \quad+\left(x_{1} \leftrightarrow x_{2}\right)+(q \leftrightarrow \bar{q}) .
\end{aligned}
$$

Here we changed variables to $z_{1}=x_{1} / x_{1}^{\prime}$.

\subsection{Comparison to resummed predictions}

We will now expand eq. (5.21) in the $\mathrm{SCET}_{\mathrm{I}}, \mathrm{SCET}_{+}$and $\mathrm{SCET}_{\mathrm{II}}$ regions of phase space, and verify that this agrees with the predictions from factorization theorems, up to power corrections. The second-to-last line of eq. (5.21) could never be produced by the factorization theorems, but is power-suppressed and does not need to be considered. Since the cross section in eq. (5.21) is a cumulative distribution, we benefit from the cumulative expressions for the ingredients of the factorization formulae in section 4 .

The minimum in eq. (5.21) cuts the $z_{1}$ interval into three regions

$$
\min \left\{\left(\frac{p_{1}^{-}}{z_{1} \mu}\right)^{2}, \frac{\mathcal{T} p_{1}^{-}}{z_{1}\left(1-z_{1}\right) \mu^{2}}, \frac{p_{T}^{2}}{\left(1-z_{1}\right)^{2} \mu^{2}}\right\}= \begin{cases}{\left[p_{1}^{-} /\left(z_{1} \mu\right)\right]^{2}} & 1 \geq z_{1} \geq z_{a} \\ \mathcal{T} p_{1}^{-} /\left[z_{1}\left(1-z_{1}\right) \mu^{2}\right] & z_{a} \geq z_{1} \geq z_{b} \\ p_{T}^{2} /\left[\left(1-z_{1}\right)^{2} \mu^{2}\right] & z_{b} \geq z_{1} \geq 0\end{cases}
$$

with $p_{1}^{-}=x_{1} E_{\mathrm{cm}}=Q e^{Y}$ and boundaries at

$$
z_{a}=\frac{1}{1+\mathcal{T} / p_{1}^{-}}, \quad z_{b}=\frac{1}{1+p_{T}^{2} /\left(\mathcal{T} p_{1}^{-}\right)}
$$

Because the size of the interval $1 \geq z_{1} \geq z_{a}$ is parametrically small, $\mathcal{O}(\mathcal{T} / Q)$, we only need to keep the logarithmically enhanced contributions. From the $z_{1} \rightarrow 1$ behavior of the splitting functions $P_{q j}\left(z_{1}\right)$ in eq. (5.12), it is clear that only the contribution from the 
diagonal $j=q$ term is not suppressed:

$$
\begin{aligned}
& \sum_{j} \int_{z_{a}}^{1} \frac{\mathrm{d} z_{1}}{z_{1}} \frac{f_{j}\left(x_{1} / z_{1}, \mu\right)}{f_{q}\left(x_{1}, \mu\right)} C_{j} P_{q j}\left(z_{1}\right) \ln \min \left\{\left(\frac{p_{1}^{-}}{z_{1} \mu}\right)^{2}, \frac{\mathcal{T} p_{1}^{-}}{z_{1}\left(1-z_{1}\right) \mu^{2}}, \frac{p_{T}^{2}}{\left(1-z_{1}\right)^{2} \mu^{2}}\right\} \\
& \quad=2 C_{F} \ln \left(\frac{p_{1}^{-}}{\mu}\right) \int_{z_{a}}^{1} \mathrm{~d} z_{1} P_{q q}\left(z_{1}\right)\left[1+\mathcal{O}\left(\frac{\mathcal{T}}{Q}\right)\right] \\
& \quad=2 C_{F} \ln \left(\frac{p_{1}^{-}}{\mu}\right)\left[-\ln \left(\frac{\left(p_{1}^{-}\right)^{2}}{\mathcal{T}^{2}}\right)+\frac{3}{2}\right]\left[1+\mathcal{O}\left(\frac{\mathcal{T}}{Q}\right)\right] \\
& \quad=C_{F}\left[-4 \ln ^{2}\left(\frac{p_{1}^{-}}{\mu}\right)+3 \ln \left(\frac{p_{1}^{-}}{\mu}\right)+4 \ln \left(\frac{p_{1}^{-}}{\mu}\right) \ln \left(\frac{\mathcal{T}}{\mu}\right)\right]\left[1+\mathcal{O}\left(\frac{\mathcal{T}}{Q}\right)\right]
\end{aligned}
$$

In the $\mathrm{SCET}_{\mathrm{I}}$ region of phase space, the interval $z_{a} \geq z_{1} \geq z_{b}$ is not parametrically small. We therefore do not give the boundary $z_{b}$ any special treatment. It is convenient to rewrite the remaining integral over $1 \geq z_{1} \geq x_{1}$ and subtract the contribution from $1 \geq z_{1} \geq z_{a}$. This requires us to extend $P_{q q}(z) \ln (1-z)$ to $z \rightarrow 1$, which we do as follows:

$$
P_{q q}(z) \ln (1-z) \rightarrow\left(1+z^{2}\right) \mathcal{L}_{1}(1-z) .
$$

We thus obtain

$$
\begin{aligned}
& \sum_{j} \int_{x_{1}}^{z_{a}} \frac{\mathrm{d} z_{1}}{z_{1}} \frac{f_{j}\left(x_{1} / z_{1}, \mu\right)}{f_{q}\left(x_{1}, \mu\right)} C_{j} P_{q j}\left(z_{1}\right) \ln \min \left\{\left(\frac{p_{1}^{-}}{z_{1} \mu}\right)^{2}, \frac{\mathcal{T} p_{1}^{-}}{z_{1}\left(1-z_{1}\right) \mu^{2}}, \frac{p_{T}^{2}}{\left(1-z_{1}\right)^{2} \mu^{2}}\right\} \\
& =\sum_{j} \int_{x_{1}}^{1} \frac{\mathrm{d} z_{1}}{z_{1}} \frac{f_{j}\left(x_{1} / z_{1}, \mu\right)}{f_{q}\left(x_{1}, \mu\right)} C_{j} P_{q j}\left(z_{1}\right)\left[\ln \min \left\{\frac{\mathcal{T} p_{1}^{-}}{z_{1} \mu^{2}}, \frac{p_{T}^{2}}{\left(1-z_{1}\right) \mu^{2}}\right\}-\ln \left(1-z_{1}\right)\right] \\
& \quad-\int_{z_{a}}^{1} \mathrm{~d} z_{1} C_{F} P_{q q}\left(z_{1}\right)\left[\ln \left(\frac{\mathcal{T} p_{1}^{-}}{\mu^{2}}\right)-\ln \left(1-z_{1}\right)\right]\left[1+\mathcal{O}\left(\frac{\mathcal{T}}{Q}\right)\right] \\
& =\sum_{j} \int_{x_{1}}^{1} \frac{\mathrm{d} z_{1}}{z_{1}} \frac{f_{j}\left(x_{1} / z_{1}, \mu\right)}{f_{q}\left(x_{1}, \mu\right)} C_{j} P_{q j}\left(z_{1}\right)\left[\ln \min \left\{\frac{\mathcal{T} p_{1}^{-}}{z_{1} \mu^{2}}, \frac{p_{T}^{2}}{(1-z) \mu^{2}}\right\}-\ln \left(1-z_{1}\right)\right] \\
& \quad+C_{F}\left[3 \ln ^{2}\left(\frac{p_{1}^{-}}{\mu}\right)-\frac{3}{2} \ln \left(\frac{p_{1}^{-}}{\mu}\right)-2 \ln \left(\frac{p_{1}^{-}}{\mu}\right) \ln \left(\frac{\mathcal{T}}{\mu}\right)-\ln ^{2}\left(\frac{\mathcal{T}}{\mu}\right)-\frac{3}{2} \ln \left(\frac{\mathcal{T}}{\mu}\right)\right]\left[1+\mathcal{O}\left(\frac{\mathcal{T}}{Q}\right)\right] .
\end{aligned}
$$

Combining eqs. (5.21), (5.24) and (5.26), it is straightforward to verify that this agrees with the $\mathrm{SCET}_{\mathrm{I}}$ factorization formula in eq. (2.9), using the results in section 4 .

In the $\mathrm{SCET}_{+}$and $\mathrm{SCET}_{\mathrm{II}}$ region of phase space, the interval $z_{a} \geq z_{1} \geq z_{b}$ is also parametrically small, $\mathcal{O}\left(p_{T}^{2} /(\mathcal{T} Q)\right)$. In fact, for $\operatorname{SCET}_{\mathrm{II}}$ both $z_{b}<z_{a}$ and $z_{b}>z_{a}$ are allowed. We start by assuming $z_{b}<z_{a}$,

$$
\begin{aligned}
& \sum_{j} \int_{z_{b}}^{z_{a}} \frac{\mathrm{d} z_{1}}{z_{1}} \frac{f_{j}\left(x_{1} / z_{1}, \mu\right)}{f_{q}\left(x_{1}, \mu\right)} C_{j} P_{q j}\left(z_{1}\right) \ln \min \left\{\left(\frac{p_{1}^{-}}{z_{1} \mu}\right)^{2}, \frac{\mathcal{T} p_{1}^{-}}{z_{1}\left(1-z_{1}\right) \mu^{2}}, \frac{p_{T}^{2}}{\left(1-z_{1}\right)^{2} \mu^{2}}\right\} \\
& =C_{F} \int_{z_{b}}^{z_{a}} \mathrm{~d} z_{1} P_{q q}\left(z_{1}\right)\left[\ln \left(\frac{\mathcal{T} p_{1}^{-}}{\mu^{2}}\right)-\ln (1-z)\right]\left[1+\mathcal{O}\left(\frac{p_{T}^{2}}{\mathcal{T} Q}\right)\right] \\
& =C_{F}\left\{-8 \ln \left(\frac{p_{1}^{-}}{\mu}\right) \ln \left(\frac{\mathcal{T}}{\mu}\right)-4 \ln ^{2}\left(\frac{\mathcal{T}}{\mu}\right)+4 \ln \left(\frac{p_{T}^{2}}{\mu^{2}}\right)\left[\ln \left(\frac{p_{1}^{-}}{\mu}\right)+\ln \left(\frac{\mathcal{T}}{\mu}\right)\right]-\ln ^{2}\left(\frac{p_{T}^{2}}{\mu^{2}}\right)\right\} \\
& \quad \times\left[1+\mathcal{O}\left(\frac{p_{T}^{2}}{\mathcal{T} Q}\right)\right] .
\end{aligned}
$$


The remainder is

$$
\begin{aligned}
\sum_{j} & \int_{x_{1}}^{z_{b}} \frac{\mathrm{d} z_{1}}{z_{1}} \frac{f_{j}\left(x_{1} / z_{1}, \mu\right)}{f_{q}\left(x_{1}, \mu\right)} C_{j} P_{q j}\left(z_{1}\right) \ln \min \left\{\left(\frac{p_{1}^{-}}{z_{1} \mu}\right)^{2}, \frac{\mathcal{T} p_{1}^{-}}{z_{1}\left(1-z_{1}\right) \mu^{2}}, \frac{p_{T}^{2}}{\left(1-z_{1}\right)^{2} \mu^{2}}\right\} \\
= & \sum_{j} \int_{x_{1}}^{1} \frac{\mathrm{d} z_{1}}{z_{1}} \frac{f_{j}\left(x_{1} / z_{1}, \mu\right)}{f_{q}\left(x_{1}, \mu\right)} C_{j} P_{q j}\left(z_{1}\right)\left[\ln \left(\frac{p_{T}^{2}}{\mu^{2}}\right)-2 \ln \left(1-z_{1}\right)\right] \\
& -\int_{z_{b}}^{1} \mathrm{~d} z_{1} C_{F} P_{q q}\left(z_{1}\right)\left[\ln \left(\frac{p_{T}^{2}}{\mu^{2}}\right)-2 \ln \left(1-z_{1}\right)\right]\left[1+\mathcal{O}\left(\frac{p_{T}^{2}}{\mathcal{T} Q}\right)\right] \\
= & \sum_{j} \int_{x_{1}}^{1} \frac{\mathrm{d} z_{1}}{z_{1}} \frac{f_{j}\left(x_{1} / z_{1}, \mu\right)}{f_{q}\left(x_{1}, \mu\right)} C_{j} P_{q j}\left(z_{1}\right)\left[\ln \left(\frac{p_{T}^{2}}{\mu^{2}}\right)-2 \ln \left(1-z_{1}\right)\right] \\
& +C_{F}\left\{2 \ln { }^{2}\left(\frac{p_{1}^{-}}{\mu}\right)+4 \ln \left(\frac{p_{1}^{-}}{\mu}\right) \ln \left(\frac{\mathcal{T}}{\mu}\right)+2 \ln ^{2}\left(\frac{\mathcal{T}}{\mu}\right)\right. \\
& \left.-2 \ln \left(\frac{p_{T}^{2}}{\mu^{2}}\right)\left[\ln \left(\frac{p_{1}^{-}}{\mu}\right)+\ln \left(\frac{\mathcal{T}}{\mu}\right)+\frac{3}{4}\right]\right\}\left[1+\mathcal{O}\left(\frac{p_{T}^{2}}{\mathcal{T} Q}\right)\right] .
\end{aligned}
$$

We have verified that this agrees with the $\mathrm{SCET}_{+}$factorization formula in eq. (2.15) expanded to NLO, providing an important check on our effective theory framework.

We now consider $z_{b}>z_{a}$, i.e. $p_{T}<\mathcal{T}$, which is only allowed by the power counting in the $\mathrm{SCET}_{\mathrm{II}}$ region of phase space. In contrast with eq. (5.22), we now only have two regions: $1 \geq z_{1} \geq z_{c}$ and $z_{c} \geq z_{1} \geq x_{1}$, where

$$
z_{c}=\frac{1}{1+p_{T} / p_{1}^{-}}
$$

This leads to the following correction to the $\mathrm{SCET}_{+}$result,

$$
\begin{aligned}
\theta\left(\mathcal{T}-p_{T}\right) & \left\{\sum_{j} \int_{z_{a}}^{z_{b}} \frac{\mathrm{d} z_{1}}{z_{1}} \frac{f_{j}\left(x_{1} / z_{1}, \mu\right)}{f_{q}\left(x_{1}, \mu\right)} C_{j} P_{q j}\left(z_{1}\right)\left[\ln \left(\frac{\mathcal{T} p_{1}^{-}}{z_{1}\left(1-z_{1}\right) \mu^{2}}\right)-\ln \left(\frac{\left(p_{1}^{-}\right)^{2}}{z_{1}^{2} \mu^{2}}\right)\right]\right. \\
+ & \left.\sum_{j} \int_{z_{b}}^{z_{c}} \frac{\mathrm{d} z_{1}}{z_{1}} \frac{f_{j}\left(x_{1} / z_{1}, \mu\right)}{f_{q}\left(x_{1}, \mu\right)} C_{j} P_{q j}\left(z_{1}\right)\left[\ln \left(\frac{p_{T}^{2}}{\left(1-z_{1}\right)^{2} \mu^{2}}\right)-\ln \left(\frac{\left(p_{1}^{-}\right)^{2}}{z_{1}^{2} \mu^{2}}\right)\right]\right\} \\
= & C_{F} \theta\left(\mathcal{T}-p_{T}\right)\left\{\int_{z_{a}}^{z_{b}} \mathrm{~d} z_{1} P_{q q}\left(z_{1}\right)\left[\ln \left(\frac{\mathcal{T}}{p_{1}^{-}}\right)-\ln \left(1-z_{1}\right)\right]\right. \\
& \left.+\int_{z_{b}}^{z_{c}} \mathrm{~d} z_{1} P_{q q}\left(z_{1}\right)\left[2 \ln \left(\frac{p_{T}}{p_{1}^{-}}\right)-2 \ln \left(1-z_{1}\right)\right]\right\}\left[1+\mathcal{O}\left(\frac{\mathcal{T}}{Q}\right)\right] \\
= & \frac{1}{2} C_{F} \theta\left(\mathcal{T}-p_{T}\right) \ln ^{2}\left(\frac{p_{T}^{2}}{\mathcal{T}^{2}}\right)\left[1+\mathcal{O}\left(\frac{\mathcal{T}}{Q}\right)\right] .
\end{aligned}
$$

The first line erases the earlier contributions from $z_{a}<z_{1}<z_{b}$ and the second line from $z_{b}<z_{1}<z_{c}$. This agrees with the FU soft function in eq. (4.10).

We conclude this section by briefly commenting on the size of the various power corrections we encountered. In section 5.2 , we restricted to $p_{T}, \mathcal{T} \ll Q$, dropping some (but not all) terms of $\mathcal{O}\left(p_{T}^{2} / Q^{2}, \mathcal{T} / Q\right)$. In our $\operatorname{SCET}_{\mathrm{I}}$ analysis in this section, we systematically expanded up to corrections of $\mathcal{O}(\mathcal{T} / Q)$. For $\operatorname{SCET}_{\mathrm{II}}$ the power corrections were 


\begin{tabular}{|l|c|}
\hline Mode: & Scaling $(-,+, \perp)$ \\
\hline collinear & $Q\left(1, \lambda^{2 r / \beta}, \lambda^{r / \beta}\right)$ \\
collinear-soft & $Q\left(\lambda^{\frac{\alpha r-\beta}{\alpha-\beta}}, \lambda^{\frac{(\alpha-2) r-(\beta-2)}{\alpha-\beta}}, \lambda^{\left.\frac{(\alpha-1) r-(\beta-1)}{\alpha-\beta}\right)}\right)$ \\
soft & $Q(\lambda, \lambda, \lambda)$ \\
\hline
\end{tabular}

Table 2. Modes and power counting in $\mathrm{SCET}_{+}$for the double angularity measurement on a single jet. The power counting parameter is $\lambda$, with $\lambda \sim e_{\alpha} \sim e_{\beta}^{1 / r}$ and $1>r>\beta / \alpha$.

$\mathcal{O}\left(\mathcal{T} / Q \sim p_{T}^{2} /(\mathcal{T} Q)\right)$, and for $\operatorname{SCET}_{+}$they were $\mathcal{O}\left(p_{T}^{2} /(\mathcal{T} Q)\right)$ in size. Contrary to our expectation in section 2.1, we found no $\mathcal{O}\left(\mathcal{T}^{2} / p_{T}^{2}\right)$ power corrections at NLO. However, it is quite possible that this changes at higher orders.

\section{Measuring two angularities on one jet}

We will now apply our effective field theory framework to the measurement of two angularities on one jet. The angularity $e_{\alpha}$ of a jet is defined as [32, 65, 102]

$$
e_{\alpha}=\sum_{i \in \text { jet }} \frac{E_{i}}{E_{\text {jet }}}\left(\frac{\theta_{i}}{R}\right)^{\alpha} .
$$

Here, $E_{i}$ and $\theta_{i}$ denote the energy and angle (with respect to the jet axis) of particle $i$, and $E_{\text {jet }}$ and $R$ are the jet energy and radius. To avoid the issue of recoil [28, 103-105], we use the winner-take-all axis $[105,106]$. This ensures that the direction of the collinear radiation coincides with the jet axis.

For the measurement of two angularities $e_{\alpha}, e_{\beta}$ (with $\alpha>\beta$ ), the phase space is given by $e_{\alpha}^{\beta / \alpha} \geq e_{\beta} \geq e_{\alpha}$ at NLL. The effective field theories on the boundaries were discussed in ref. [34], so we focus on the intermediate regime described by $\mathrm{SCET}_{+}$. The modes of $\mathrm{SCET}_{+}$are shown in table 2 . Their power counting is fixed by the requirement that these modes are on-shell, that the collinear mode contributes to $e_{\beta}$, the soft mode contribute to $e_{\alpha}$ and the collinear-soft mode contribute to both. This leads to the following factorization formula

$$
\begin{aligned}
\frac{\mathrm{d}^{2} \sigma_{i}}{\mathrm{~d} e_{\alpha} \mathrm{d} e_{\beta}}= & \hat{\sigma}_{i}^{(0)} H_{i}\left(Q^{2}, \mu\right) \int \mathrm{d} e_{\beta}^{\mathrm{c}} Q^{\beta} \mathrm{d} e_{\alpha}^{\mathrm{cs}} Q \mathrm{~d} e_{\beta}^{\mathrm{cs}} Q^{\beta} \mathrm{d} e_{\alpha}^{\mathrm{s}} Q J_{i}\left(e_{\beta}^{\mathrm{c}} Q^{\beta}, \mu\right) \mathscr{S}_{i}\left(e_{\alpha}^{\mathrm{cs}} Q, e_{\beta}^{\mathrm{cs}} Q^{\beta}\right) S_{i}\left(e_{\alpha}^{\mathrm{s}} Q, \mu\right) \\
& \times \delta\left(e_{\alpha}-e_{\alpha}^{\mathrm{cs}}-e_{\alpha}^{\mathrm{c}}\right) \delta\left(e_{\beta}-e_{\beta}^{\mathrm{c}}-e_{\beta}^{\mathrm{cs}}\right)
\end{aligned}
$$

for quark $(i=q)$ and gluon $(i=g)$ jets. Here, $\hat{\sigma}_{i}^{(0)}$ is the tree-level cross section, and $H$ the hard function describing hard virtual corrections. The jet function $J$, soft function $S$ and collinear-soft function $\mathscr{S}$ capture the effect of collinear, soft and collinear-soft radiation, respectively. The first two have been defined in ref. [34] while the third is the analog of eq. (2.17) but for the double angularity measurement. Since we only work up to NLL order, we are allowed to consider a single jet. At higher orders we need to take the rest of the event into account, and eq. (6.2) must accordingly be generalized to e.g. $e^{+} e^{-}$event 
shapes. We expect the power corrections to be $\mathcal{O}\left(e_{\alpha} / e_{\beta}, e_{\beta}^{\alpha / \beta} / e_{\alpha}\right)$, which blow up at the edges of the phase space, where the boundary theories should be used instead.

Below we collect what is needed for NLL resummation. The RG equation and the anomalous dimension of the hard function are

$$
\begin{aligned}
\mu \frac{\mathrm{d}}{\mathrm{d} \mu} H_{i}\left(Q^{2}, \mu\right) & =\gamma_{H}^{i}\left(Q^{2}, \mu\right) H_{i}\left(Q^{2}, \mu\right), \\
\gamma_{H}^{i}\left(Q^{2}, \mu\right) & =\Gamma_{\text {cusp }}^{i}\left(\alpha_{s}\right) \ln \frac{Q^{2}}{\mu^{2}}+\gamma_{H}^{i}\left(\alpha_{s}\right) .
\end{aligned}
$$

For the jet function we have

$$
\begin{aligned}
\mu \frac{\mathrm{d}}{\mathrm{d} \mu} J_{i}\left(e_{\beta} Q^{\beta}, \mu\right) & =\int_{0}^{e_{\beta}} \mathrm{d} e_{\beta}^{\prime} Q^{\beta} \gamma_{J}^{i}\left(e_{\beta} Q^{\beta}-e_{\beta}^{\prime} Q^{\beta}, \mu\right) J_{i}\left(e_{\beta}^{\prime} Q^{\beta}, \mu\right), \\
\gamma_{J}^{i}\left(e_{\beta} Q^{\beta}, \mu\right) & =-\frac{2}{\beta-1} \Gamma_{\text {cusp }}^{i}\left(\alpha_{s}\right) \frac{1}{\mu^{\beta}} \mathcal{L}_{0}\left(\frac{e_{\beta} Q^{\beta}}{\mu^{\beta}}\right)+\gamma_{J}^{i}\left(\alpha_{s}\right) \delta\left(e_{\beta} Q^{\beta}\right),
\end{aligned}
$$

and the soft function satisfies

$$
\begin{aligned}
\mu \frac{\mathrm{d}}{\mathrm{d} \mu} S_{i}\left(e_{\alpha} Q, \mu\right) & =\int_{0}^{e_{\alpha}} \mathrm{d} e_{\alpha}^{\prime} Q \gamma_{S}^{i}\left(e_{\alpha} Q-e_{\alpha}^{\prime} Q, \mu\right) S_{i}\left(e_{\alpha}^{\prime} Q, \mu\right) \\
\gamma_{S}^{i}\left(e_{\alpha} Q, \mu\right) & =\frac{2}{\alpha-1} \Gamma_{\text {cusp }}^{i}\left(\alpha_{s}\right) \frac{1}{\mu} \mathcal{L}_{0}\left(\frac{e_{\alpha} Q}{\mu}\right)+\gamma_{S}^{i}\left(\alpha_{s}\right) \delta\left(e_{\alpha} Q\right) .
\end{aligned}
$$

The anomalous dimension of the collinear-soft function is constrained by consistency of the cross section in eq. (6.2). These anomalous dimensions involve $\Gamma_{\text {cusp }}^{i}\left(\alpha_{s}\right)$, given in appendix $\mathrm{B}$, and the non-cusp parts

$$
\gamma_{X}^{i}\left(\alpha_{s}\right)=\sum_{n} \gamma_{X, n}^{i}\left(\frac{\alpha_{s}}{4 \pi}\right)^{n+1},
$$

with $X=H, J, S$. At NLL we only need the leading coefficients,

$$
\gamma_{H, 0}^{q}=-6 C_{F}, \quad \gamma_{H, 0}^{g}=-2 \beta_{0}, \quad \gamma_{J, 0}^{i}=-\gamma_{H, 0}^{i}, \quad \gamma_{S, 0}^{i}=0,
$$

where $\beta_{0}=\frac{11}{3} C_{A}-\frac{4}{3} T_{F} n_{f}$.

We now evaluate the double cumulative distribution at NLL order by inserting the tree-level expressions

$$
\begin{aligned}
H_{i}\left(Q^{2}, \mu\right) & =1, & J_{i}\left(e_{\beta} Q^{\beta}, \mu\right) & =\delta\left(e_{\beta} Q^{\beta}\right), \\
\mathscr{S}_{i}\left(e_{\alpha} Q, e_{\beta} Q^{\beta}\right) & =\delta\left(e_{\alpha} Q\right) \delta\left(e_{\beta} Q^{\beta}\right), & S_{i}\left(e_{\alpha} Q, \mu\right) & =\delta\left(e_{\alpha} Q\right),
\end{aligned}
$$

in eq. (6.2) and evolving them to the collinear-soft scale $\mu_{\mathscr{S}}$. This results in

$$
\begin{aligned}
\Sigma_{i}\left(e_{\alpha}, e_{\beta}\right) & =\int_{0}^{e_{\alpha}} \mathrm{d} e_{\alpha}^{\prime} \int_{0}^{e_{\beta}} \mathrm{d} e_{\beta}^{\prime} \frac{\partial^{2} \sigma}{\partial e_{\alpha}^{\prime} \partial e_{\beta}^{\prime}} \\
& =\hat{\sigma}_{i}^{(0)} \frac{e^{K_{H}^{i}+K_{J}^{i}+K_{S}^{i}-\gamma_{E} \eta_{J}^{i}-\gamma_{E}} \eta_{S}^{i}}{\Gamma\left(1+\eta_{J}^{i}\right) \Gamma\left(1+\eta_{S}^{i}\right)}\left(\frac{Q}{\mu_{H}}\right)^{2 \eta_{H}^{i}}\left(\frac{e_{\beta}^{1 / \beta} Q}{\mu_{J}}\right)^{\beta \eta_{J}^{i}}\left(\frac{e_{\alpha} Q}{\mu_{S}}\right)^{\eta_{S}^{i}} .
\end{aligned}
$$


The evolution kernels that enter here are

$$
\begin{aligned}
K_{H}^{i}\left(\mu_{H}, \mu_{\mathscr{S}}\right) & =-2 K_{\Gamma}^{i}\left(\mu_{H}, \mu_{\mathscr{S}}\right)+K_{\gamma_{H}^{i}}\left(\mu_{H}, \mu_{\mathscr{S}}\right), & \eta_{H}^{i}\left(\mu_{J}, \mu_{\mathscr{S}}\right) & =\eta_{\Gamma}^{i}\left(\mu_{J}, \mu_{\mathscr{S}}\right), \quad(6.10) \\
K_{J}^{i}\left(\mu_{J}, \mu_{\mathscr{S}}\right) & =-\frac{2 \beta}{1-\beta} K_{\Gamma}^{i}\left(\mu_{J}, \mu_{\mathscr{S}}\right)+K_{\gamma_{J}^{i}}\left(\mu_{J}, \mu_{\mathscr{S}}\right), & \eta_{J}^{i}\left(\mu_{J}, \mu_{\mathscr{S}}\right) & =\frac{2}{1-\beta} \eta_{\Gamma}^{i}\left(\mu_{J}, \mu_{\mathscr{S}}\right), \\
K_{S}^{i}\left(\mu_{S}, \mu_{\mathscr{S}}\right) & =\frac{2}{1-\alpha} K_{\Gamma}^{i}\left(\mu_{S}, \mu_{\mathscr{S}}\right), & \eta_{S}^{i}\left(\mu_{S}, \mu_{\mathscr{S}}\right) & =-\frac{2}{1-\alpha} \eta_{\Gamma}^{i}\left(\mu_{S}, \mu_{\mathscr{S}}\right),
\end{aligned}
$$

in terms of $K_{\Gamma}^{i}, \eta_{\Gamma}^{i}$ and $K_{\gamma_{X}^{i}}$ defined in eq. (B.1). As starting point for the RG evolution we use the canonical (natural) scales

$$
\begin{aligned}
\mu_{H} & =Q, \\
\mu_{J} & =e_{\beta}^{1 / \beta} Q=\mu_{J \rightarrow J}, \\
\mu_{\mathscr{S}} & =\left(e_{\alpha}^{1-\beta} e_{\beta}^{\alpha-1}\right)^{1 /(\alpha-\beta)} Q=\mu_{J \rightarrow S}, \\
\mu_{S} & =e_{\alpha} Q=\mu_{S \rightarrow S} .
\end{aligned}
$$

which we identified with the interpolating scales $\mu_{J \rightarrow J}, \mu_{J \rightarrow S}$ and $\mu_{S \rightarrow S}$ of ref. [34] (see also appendix $\mathrm{C}$ of ref. [37]) to simplify the comparison.

This mostly agrees with the conjecture made in ref. [34]

$$
\Sigma_{i}^{\text {ref. }[34]}\left(e_{\alpha}, e_{\beta}\right)=\frac{e^{-R\left(e_{\alpha}, e_{\beta}\right)-\gamma_{E} \tilde{R}\left(e_{\alpha}, e_{\beta}\right)}}{\Gamma\left(1+\tilde{R}\left(e_{\alpha}, e_{\beta}\right)\right)}
$$

where

$$
\begin{aligned}
& R\left(e_{\alpha}, e_{\beta}\right) \stackrel{\mathrm{NLL}}{=}-K_{H}^{i}\left(\mu_{H}, \mu_{\mathscr{S}}\right)-K_{J}^{i}\left(\mu_{J}, \mu_{\mathscr{S}}\right)-K_{S}^{i}\left(\mu_{S}, \mu_{\mathscr{S}}\right), \\
& \tilde{R}\left(e_{\alpha}, e_{\beta}\right) \stackrel{\mathrm{NLL}}{=} \eta_{J}^{i}\left(\mu_{J}, \mu_{\mathscr{S}}\right)+\eta_{S}^{i}\left(\mu_{S}, \mu_{\mathscr{S}}\right) .
\end{aligned}
$$

The only difference ${ }^{12}$ with our result in eq. (6.9) is in the denominator, where we have $\Gamma\left(1+\eta_{J}^{i}\right) \Gamma\left(1+\eta_{S}^{i}\right)$ instead of $\Gamma\left(1+\eta_{J}^{i}+\eta_{S}^{i}\right)$. These factors agree with each other on the boundary, since either $\eta_{J}^{i}$ or $\eta_{S}^{i}$ vanishes there, but lead to $\mathcal{O}\left(\alpha_{s}^{2} \ln ^{2}\right)$ differences in the bulk. (An analogous conjecture to eq. (6.12) in Laplace space does agree with our result. ${ }^{13}$ )

According to ref. [34], the leading difference between their interpolation and the true NLL cross section is expected to be $\alpha_{s}^{4} \ln ^{4}$. However, this is based on boundary conditions for the differential cross section, which do not affect the logarithmic accuracy of their calculation in the bulk. Specifically, their differential cross section satisfies the condition at the boundary $e_{\alpha}=e_{\beta}^{\alpha / \beta}$ through the addition of terms that are power suppressed. Since these terms are power suppressed in the bulk, they cannot improve the logarithmic accuracy there.

In ref. [101], we will discuss how a more sophisticated scale choice than eq. (6.11) provides a natural way to satisfy the derivative boundary condition. In addition to requiring

\footnotetext{
${ }^{12}$ Ignoring differences beyond NLL order and power suppressed contributions.

${ }^{13}$ We thank D. Neill for pointing this out.
} 
$\mu_{\mathscr{S}}$ to merge with $\mu_{J}$ or $\mu_{S}$ on the respective boundaries, one can also require a continuous derivative,

$$
\begin{aligned}
\left.\frac{\partial}{\partial e_{\alpha}} \mu_{J}\left(e_{\alpha}, e_{\beta}\right)\right|_{e_{\beta}=e_{\alpha}^{\beta / \alpha}}=\frac{\mathrm{d}}{\mathrm{d} e_{\alpha}} \mu_{J}\left(e_{\alpha}, e_{\alpha}^{\beta / \alpha}\right), & \left.\frac{\partial}{\partial e_{\beta}} \mu_{J}\left(e_{\alpha}, e_{\beta}\right)\right|_{e_{\beta}=e_{\alpha}^{\beta / \alpha}}=0, \\
\left.\frac{\partial}{\partial e_{\alpha}} \mu_{\mathscr{S}}\left(e_{\alpha}, e_{\beta}\right)\right|_{e_{\beta}=e_{\alpha}^{\beta / \alpha}}=\frac{\mathrm{d}}{\mathrm{d} e_{\alpha}} \mu_{J}\left(e_{\alpha}, e_{\alpha}^{\beta / \alpha}\right), & \left.\frac{\partial}{\partial e_{\beta}} \mu_{\mathscr{S}}\left(e_{\alpha}, e_{\beta}\right)\right|_{e_{\beta}=e_{\alpha}^{\beta / \alpha}}=0, \\
\left.\frac{\partial}{\partial e_{\alpha}} \mu_{S}\left(e_{\alpha}, e_{\beta}\right)\right|_{e_{\beta}=e_{\alpha}^{\beta / \alpha}}=\frac{\mathrm{d}}{\mathrm{d} e_{\alpha}} \mu_{S}\left(e_{\alpha}, e_{\alpha}^{\beta / \alpha}\right), & \left.\frac{\partial}{\partial e_{\beta}} \mu_{S}\left(e_{\alpha}, e_{\beta}\right)\right|_{e_{\beta}=e_{\alpha}^{\beta / \alpha}}=0,
\end{aligned}
$$

and similarly for the boundary at $e_{\alpha}=e_{\beta}$. These equations closely resemble those imposed on $R$ and $\tilde{R}$ in ref. [34] and follow from the same steps. Note that there is a redundancy in the constraints in eq. (6.14), as e.g. the second equation on the first line implies the first. The scale choice in transitioning to a region where resummation is turned off has been studied for single variables in e.g. refs. [97, 100], and also in ref. [52].

\section{Conclusions}

In this paper we studied the resummation of double differential measurements. We focussed on two examples: Drell-Yan production with a (beam-thrust) jet veto where the $p_{T}$ of the lepton pair is measured, and the measurement of two angularities on one jet. Concerning the latter, in ref. [34] resummation on the two phase space boundaries was achieved, and an interpolation was built to smoothly connect them. We go beyond this by identifying the factorization formula needed to achieve resummation in the intermediate regime. This involves additional collinear-soft modes, and the corresponding collinear-soft function was calculated at one loop. The relations between FU PDFs, collinear-soft functions and (FU) soft functions were investigated. The consistency of our factorization theorem was verified by checking that the anomalous dimensions cancel between the various ingredients, and by comparing to an analytic NLO calculation of the cross section. We also showed how to combine the factorization theorems on the boundaries and interior, to achieve NNLL precision throughout. At variance with ref. [34] we found a universal factorization formula that describes the cross section in all three phase space regions up to power corrections. Numerical results, including the matching to fixed order, will be presented in ref. [101].

If the hierarchy of scales for the individual variables is not that large, such that the resummation of them is only marginally important, there may be not enough room for a distinct $\mathrm{SCET}_{+}$region of phase space. (This can be seen in figure 1, where you have to go deeper into the resummation region for $\mathrm{SCET}_{+}$.) Even in this case, one benefits from knowing the correct description of the intermediate regime in building the interpolation between boundaries, as illustrated by the $\mathcal{O}\left(\alpha_{s}^{2} \ln ^{2}\right)$ difference between our NLL results and the interpolation conjectured in ref. [34].

Looking forward, we hope the results presented here will stimulate the development of more realistic analytic resummations and more robust Monte Carlo descriptions of LHC events. The framework presented here has natural generalizations to resummation in more than two variables. Finally, finding a proper description of the "terra incognita" in figure 1 
is important for resolving a long-standing issue over double counting between higher-order corrections and double parton scatterings.

\section{Acknowledgments}

We thank Thomas Becher and Mathias Ritzmann for discussions. We thank Andrew Larkoski, Ian Moult, Duff Neil, Frank Tackmann and Jonathan Walsh for feedback on this manuscript. W.W. thanks the Galileo Galilei Institute for Theoretical Physics for hospitality and the INFN for partial support during the completion of this work. M.P. acknowledges support by the Swiss National Science Foundation. W.W. is supported by a Marie Curie International Incoming Fellowship within the 7th European Community Framework Program (PIIF-GA-2012-328913). This work is part of the D-ITP consortium, a program of the Netherlands Organization for Scientific Research (NWO) that is funded by the Dutch Ministry of Education, Culture and Science (OCW).

\section{A Plus distributions}

The standard plus distribution for some function $g(x)$ can be defined as

$$
[\theta(x) g(x)]_{+}=\lim _{\beta \rightarrow 0} \frac{\mathrm{d}}{\mathrm{d} x}[\theta(x-\beta) G(x)] \quad \text { with } \quad G(x)=\int_{1}^{x} \mathrm{~d} x^{\prime} g\left(x^{\prime}\right),
$$

satisfying the boundary condition $\int_{0}^{1} \mathrm{~d} x[\theta(x) g(x)]_{+}=0$. Two special cases we need are

$$
\begin{aligned}
& \mathcal{L}_{n}(x) \equiv\left[\frac{\theta(x) \ln ^{n} x}{x}\right]_{+}=\lim _{\beta \rightarrow 0}\left[\frac{\theta(x-\beta) \ln ^{n} x}{x}+\delta(x-\beta) \frac{\ln ^{n+1} \beta}{n+1}\right], \\
& \mathcal{L}^{\eta}(x) \equiv\left[\frac{\theta(x)}{x^{1-\eta}}\right]_{+}=\lim _{\beta \rightarrow 0}\left[\frac{\theta(x-\beta)}{x^{1-\eta}}+\delta(x-\beta) \frac{x^{\eta}-1}{\eta}\right] .
\end{aligned}
$$

In our calculations, we use the following expansion in plus distributions

$$
\frac{\theta(x)}{x^{1+\epsilon}}=-\frac{1}{\epsilon} \delta(x)+\mathcal{L}_{0}(x)-\epsilon \mathcal{L}_{1}(x)+\mathcal{O}\left(\epsilon^{2}\right) .
$$

Rescaling and convolution identities for $\mathcal{L}_{n}(x)$ and $\mathcal{L}^{\eta}(x)$ can be found in appendix B of ref. [97].

\section{B Renormalization group evolution}

The functions $K_{\Gamma}^{i}\left(\mu_{0}, \mu\right), \eta_{\Gamma}^{i}\left(\mu_{0}, \mu\right)$ and $K_{\gamma_{X}^{i}}\left(\mu_{0}, \mu\right)$ that enter in the RGE solutions are defined by

$$
\begin{aligned}
K_{\Gamma}^{i}\left(\mu_{0}, \mu\right) & =\int_{\alpha_{s}\left(\mu_{0}\right)}^{\alpha_{s}(\mu)} \frac{\mathrm{d} \alpha_{s}}{\beta\left(\alpha_{s}\right)} \Gamma_{\text {cusp }}^{i}\left(\alpha_{s}\right) \int_{\alpha_{s}\left(\mu_{0}\right)}^{\alpha_{s}} \frac{\mathrm{d} \alpha_{s}^{\prime}}{\beta\left(\alpha_{s}^{\prime}\right)}, \quad \eta_{\Gamma}^{i}\left(\mu_{0}, \mu\right)=\int_{\alpha_{s}\left(\mu_{0}\right)}^{\alpha_{s}(\mu)} \frac{\mathrm{d} \alpha_{s}}{\beta\left(\alpha_{s}\right)} \Gamma_{\text {cusp }}^{i}\left(\alpha_{s}\right), \\
K_{\gamma_{X}^{i}}\left(\mu_{0}, \mu\right) & =\int_{\alpha_{s}\left(\mu_{0}\right)}^{\alpha_{s}(\mu)} \frac{\mathrm{d} \alpha_{s}}{\beta\left(\alpha_{s}\right)} \gamma_{X}^{i}\left(\alpha_{s}\right) .
\end{aligned}
$$


Expanding the beta function and anomalous dimensions in powers of $\alpha_{s}$,

$$
\begin{aligned}
\beta\left(\alpha_{s}\right) & =-2 \alpha_{s} \sum_{n=0}^{\infty} \beta_{n}\left(\frac{\alpha_{s}}{4 \pi}\right)^{n+1}, \quad \Gamma_{\text {cusp }}^{i}\left(\alpha_{s}\right)=\sum_{n=0}^{\infty} \Gamma_{n}^{i}\left(\frac{\alpha_{s}}{4 \pi}\right)^{n+1}, \\
\gamma_{X}^{i}\left(\alpha_{s}\right) & =\sum_{n=0}^{\infty} \gamma_{X, n}^{i}\left(\frac{\alpha_{s}}{4 \pi}\right)^{n+1},
\end{aligned}
$$

their explicit expressions at NNLL order are

$$
\begin{aligned}
K_{\Gamma}^{i}\left(\mu_{0}, \mu\right)= & -\frac{\Gamma_{0}^{i}}{4 \beta_{0}^{2}}\left\{\frac{4 \pi}{\alpha_{s}\left(\mu_{0}\right)}\left(1-\frac{1}{r}-\ln r\right)+\left(\frac{\Gamma_{1}^{i}}{\Gamma_{0}^{i}}-\frac{\beta_{1}}{\beta_{0}}\right)(1-r+\ln r)+\frac{\beta_{1}}{2 \beta_{0}} \ln ^{2} r\right. \\
& +\frac{\alpha_{s}\left(\mu_{0}\right)}{4 \pi}\left[\left(\frac{\beta_{1}^{2}}{\beta_{0}^{2}}-\frac{\beta_{2}}{\beta_{0}}\right)\left(\frac{1-r^{2}}{2}+\ln r\right)+\left(\frac{\beta_{1} \Gamma_{1}^{i}}{\beta_{0} \Gamma_{0}^{i}}-\frac{\beta_{1}^{2}}{\beta_{0}^{2}}\right)(1-r+r \ln r)\right. \\
& \left.\left.-\left(\frac{\Gamma_{2}^{i}}{\Gamma_{0}^{i}}-\frac{\beta_{1} \Gamma_{1}^{i}}{\beta_{0} \Gamma_{0}^{i}}\right) \frac{(1-r)^{2}}{2}\right]\right\} \\
\eta_{\Gamma}^{i}\left(\mu_{0}, \mu\right)= & -\frac{\Gamma_{0}^{i}}{2 \beta_{0}}\left[\ln r+\frac{\alpha_{s}\left(\mu_{0}\right)}{4 \pi}\left(\frac{\Gamma_{1}^{i}}{\Gamma_{0}^{i}}-\frac{\beta_{1}}{\beta_{0}}\right)(r-1)\right. \\
& \left.+\frac{\alpha_{s}^{2}\left(\mu_{0}\right)}{16 \pi^{2}}\left(\frac{\Gamma_{2}^{i}}{\Gamma_{0}^{i}}-\frac{\beta_{1} \Gamma_{1}^{i}}{\beta_{0} \Gamma_{0}^{i}}+\frac{\beta_{1}^{2}}{\beta_{0}^{2}}-\frac{\beta_{2}}{\beta_{0}}\right) \frac{r^{2}-1}{2}\right] \\
K_{\gamma_{X}^{i}}\left(\mu_{0}, \mu\right)= & -\frac{\gamma_{X, 0}^{i}}{2 \beta_{0}}\left[\ln r+\frac{\alpha_{s}\left(\mu_{0}\right)}{4 \pi}\left(\frac{\gamma_{X, 1}^{i}}{\gamma_{X, 0}^{i}}-\frac{\beta_{1}}{\beta_{0}}\right)(r-1)\right] .
\end{aligned}
$$

Here, $r=\alpha_{s}(\mu) / \alpha_{s}\left(\mu_{0}\right)$ and the running coupling is given by the three-loop expression

$$
\frac{1}{\alpha_{s}(\mu)}=\frac{X}{\alpha_{s}\left(\mu_{0}\right)}+\frac{\beta_{1}}{4 \pi \beta_{0}} \ln X+\frac{\alpha_{s}\left(\mu_{0}\right)}{16 \pi^{2}}\left[\frac{\beta_{2}}{\beta_{0}}\left(1-\frac{1}{X}\right)+\frac{\beta_{1}^{2}}{\beta_{0}^{2}}\left(\frac{\ln X}{X}+\frac{1}{X}-1\right)\right],
$$

where $X=1+\alpha_{s}\left(\mu_{0}\right) \beta_{0} \ln \left(\mu / \mu_{0}\right) /(2 \pi)$.

The coefficients of the beta function [107, 108], cusp anomalous dimension [109, 110], non-cusp anomalous dimensions of the hard function and jet function [1, 110-117] and noncusp anomalous dimension of the rapidity resummation $[10,13,14,118]$ are given below in the $\overline{\mathrm{MS}}$ scheme. At this order $\Gamma_{i}^{g}=\left(C_{A} / C_{F}\right) \Gamma_{i}^{q}$, which are therefore not separately shown.

$$
\begin{aligned}
& \beta_{0}= \frac{11}{3} C_{A}-\frac{4}{3} T_{F} n_{f}, \\
& \beta_{1}= \frac{34}{3} C_{A}^{2}-\left(\frac{20}{3} C_{A}+4 C_{F}\right) T_{F} n_{f}, \\
& \beta_{2}=\frac{2857}{54} C_{A}^{3}+\left(C_{F}^{2}-\frac{205}{18} C_{F} C_{A}-\frac{1415}{54} C_{A}^{2}\right) 2 T_{F} n_{f}+\left(\frac{11}{9} C_{F}+\frac{79}{54} C_{A}\right) 4 T_{F}^{2} n_{f}^{2}, \\
& \Gamma_{0}^{q}=4 C_{F}, \\
& \Gamma_{1}^{q}=4 C_{F}\left[\left(\frac{67}{9}-\frac{\pi^{2}}{3}\right) C_{A}-\frac{20}{9} T_{F} n_{f}\right] \\
& \Gamma_{2}^{q}=4 C_{F}\left[\left(\frac{245}{6}-\frac{134 \pi^{2}}{27}+\frac{11 \pi^{4}}{45}+\frac{22 \zeta_{3}}{3}\right) C_{A}^{2}+\left(-\frac{418}{27}+\frac{40 \pi^{2}}{27}-\frac{56 \zeta_{3}}{3}\right) C_{A} T_{F} n_{f}\right. \\
&\left.\quad+\left(-\frac{55}{3}+16 \zeta_{3}\right) C_{F} T_{F} n_{f}-\frac{16}{27} T_{F}^{2} n_{f}^{2}\right]
\end{aligned}
$$




$$
\begin{aligned}
\gamma_{H 0}^{q}=-6 C_{F}, & \\
\gamma_{H 1}^{q}=-C_{F}[ & \left.\left(\frac{82}{9}-52 \zeta_{3}\right) C_{A}+\left(3-4 \pi^{2}+48 \zeta_{3}\right) C_{F}+\left(\frac{65}{9}+\pi^{2}\right) \beta_{0}\right] \\
\gamma_{H 2}^{q}=-2 C_{F}[ & \left(\frac{66167}{324}-\frac{686 \pi^{2}}{81}-\frac{302 \pi^{4}}{135}-\frac{782 \zeta_{3}}{9}+\frac{44 \pi^{2} \zeta_{3}}{9}+136 \zeta_{5}\right) C_{A}^{2} \\
& +\left(\frac{151}{4}-\frac{205 \pi^{2}}{9}-\frac{247 \pi^{4}}{135}+\frac{844 \zeta_{3}}{3}+\frac{8 \pi^{2} \zeta_{3}}{3}+120 \zeta_{5}\right) C_{F} C_{A} \\
& +\left(\frac{29}{2}+3 \pi^{2}+\frac{8 \pi^{4}}{5}+68 \zeta_{3}-\frac{16 \pi^{2} \zeta_{3}}{3}-240 \zeta_{5}\right) C_{F}^{2} \\
& +\left(-\frac{10781}{108}+\frac{446 \pi^{2}}{81}+\frac{449 \pi^{4}}{270}-\frac{1166 \zeta_{3}}{9}\right) C_{A} \beta_{0} \\
& \left.+\left(\frac{2953}{108}-\frac{13 \pi^{2}}{18}-\frac{7 \pi^{4}}{27}+\frac{128 \zeta_{3}}{9}\right) \beta_{1}+\left(-\frac{2417}{324}+\frac{5 \pi^{2}}{6}+\frac{2 \zeta_{3}}{3}\right) \beta_{0}^{2}\right]
\end{aligned}
$$

$\gamma_{H 0}^{g}=-2 \beta_{0}$,

$$
\begin{aligned}
\gamma_{H 1}^{g}= & \left(-\frac{118}{9}+4 \zeta_{3}\right) C_{A}^{2}+\left(-\frac{38}{9}+\frac{\pi^{2}}{3}\right) C_{A} \beta_{0}-2 \beta_{1}, \\
\gamma_{H 2}^{g}= & \left(-\frac{60875}{162}+\frac{634 \pi^{2}}{81}+\frac{8 \pi^{4}}{5}+\frac{1972 \zeta_{3}}{9}-\frac{40 \pi^{2} \zeta_{3}}{9}-32 \zeta_{5}\right) C_{A}^{3} \\
& +\left(\frac{7649}{54}+\frac{134 \pi^{2}}{81}-\frac{61 \pi^{4}}{45}-\frac{500 \zeta_{3}}{9}\right) C_{A}^{2} \beta_{0}+\left(\frac{466}{81}+\frac{5 \pi^{2}}{9}-\frac{28 \zeta_{3}}{3}\right) C_{A} \beta_{0}^{2} \\
& +\left(-\frac{1819}{54}+\frac{\pi^{2}}{3}+\frac{4 \pi^{4}}{45}+\frac{152 \zeta_{3}}{9}\right) C_{A} \beta_{1}-2 \beta_{2},
\end{aligned}
$$

$$
\begin{aligned}
& \gamma_{J 0}^{q}=6 C_{F}, \\
& \gamma_{J 1}^{q}=C_{F}\left[\left(\frac{146}{9}-80 \zeta_{3}\right) C_{A}+\left(3-4 \pi^{2}+48 \zeta_{3}\right) C_{F}+\left(\frac{121}{9}+\frac{2 \pi^{2}}{3}\right) \beta_{0}\right],
\end{aligned}
$$$$
\gamma_{J 2}^{q}=2 C_{F}\left[\left(\frac{52019}{162}-\frac{841 \pi^{2}}{81}-\frac{82 \pi^{4}}{27}-\frac{2056 \zeta_{3}}{9}+\frac{88 \pi^{2} \zeta_{3}}{9}+232 \zeta_{5}\right) C_{A}^{2}\right.
$$$$
+\left(\frac{151}{4}-\frac{205 \pi^{2}}{9}-\frac{247 \pi^{4}}{135}+\frac{844 \zeta_{3}}{3}+\frac{8 \pi^{2} \zeta_{3}}{3}+120 \zeta_{5}\right) C_{A} C_{F}
$$$$
+\left(\frac{29}{2}+3 \pi^{2}+\frac{8 \pi^{4}}{5}+68 \zeta_{3}-\frac{16 \pi^{2} \zeta_{3}}{3}-240 \zeta_{5}\right) C_{F}^{2}
$$$$
+\left(-\frac{7739}{54}+\frac{325}{81} \pi^{2}+\frac{617 \pi^{4}}{270}-\frac{1276 \zeta_{3}}{9}\right) C_{A} \beta_{0}
$$$$
\left.+\left(-\frac{3457}{324}+\frac{5 \pi^{2}}{9}+\frac{16 \zeta_{3}}{3}\right) \beta_{0}^{2}+\left(\frac{1166}{27}-\frac{8 \pi^{2}}{9}-\frac{41 \pi^{4}}{135}+\frac{52 \zeta_{3}}{9}\right) \beta_{1}\right],
$$

$\gamma_{J 0}^{g}=2 \beta_{0}$,

$\gamma_{J 1}^{g}=\left(\frac{182}{9}-32 \zeta_{3}\right) C_{A}^{2}+\left(\frac{94}{9}-\frac{2 \pi^{2}}{3}\right) C_{A} \beta_{0}+2 \beta_{1}$, 


$$
\begin{aligned}
\gamma_{J 2}^{g}= & \left(\frac{49373}{81}-\frac{944 \pi^{2}}{81}-\frac{16 \pi^{4}}{5}-\frac{4520 \zeta_{3}}{9}+\frac{128 \pi^{2} \zeta_{3}}{9}+224 \zeta_{5}\right) C_{A}^{3} \\
& +\left(-\frac{6173}{27}-\frac{376 \pi^{2}}{81}+\frac{13 \pi^{4}}{5}+\frac{280 \zeta_{3}}{9}\right) C_{A}^{2} \beta_{0}+\left(-\frac{986}{81}-\frac{10 \pi^{2}}{9}+\frac{56 \zeta_{3}}{3}\right) C_{A} \beta_{0}^{2} \\
& +\left(\frac{1765}{27}-\frac{2 \pi^{2}}{3}-\frac{8 \pi^{4}}{45}-\frac{304 \zeta_{3}}{9}\right) C_{A} \beta_{1}+2 \beta_{2} \\
\gamma_{\nu, 0}^{q}= & 0 \\
\gamma_{\nu, 1}^{q}= & C_{F}\left[\left(\frac{64}{9}-28 \zeta_{3}\right) C_{A}+32 \zeta_{3} C_{F}+\frac{56}{9} \beta_{0}\right] \\
\gamma_{\nu, 0}^{g}= & 0 \\
\gamma_{\nu, 1}^{g}= & C_{A}\left[\left(\frac{64}{9}+4 \zeta_{3}\right) C_{A}+\frac{56}{9} \beta_{0}\right]
\end{aligned}
$$

Open Access. This article is distributed under the terms of the Creative Commons Attribution License (CC-BY 4.0), which permits any use, distribution and reproduction in any medium, provided the original author(s) and source are credited.

\section{References}

[1] C.F. Berger, C. Marcantonini, I.W. Stewart, F.J. Tackmann and W.J. Waalewijn, Higgs Production with a Central Jet Veto at NNLL+NNLO, JHEP 04 (2011) 092 [arXiv: 1012.4480] [INSPIRE].

[2] A. Banfi, P.F. Monni, G.P. Salam and G. Zanderighi, Higgs and Z-boson production with a jet veto, Phys. Rev. Lett. 109 (2012) 202001 [arXiv:1206.4998] [InSPIRE].

[3] X. Liu and F. Petriello, Reducing theoretical uncertainties for exclusive Higgs-boson plus one-jet production at the LHC, Phys. Rev. D 87 (2013) 094027 [arXiv:1303.4405] [INSPIRE].

[4] T. Becher, M. Neubert and L. Rothen, Factorization and $N^{3} L L_{p}+N N L O$ predictions for the Higgs cross section with a jet veto, JHEP 10 (2013) 125 [arXiv:1307.0025] [INSPIRE].

[5] I.W. Stewart, F.J. Tackmann, J.R. Walsh and S. Zuberi, Jet $p_{T}$ resummation in Higgs production at $N N L L^{\prime}+N N L O$, Phys. Rev. D 89 (2014) 054001 [arXiv:1307.1808] [INSPIRE].

[6] R. Boughezal, X. Liu, F. Petriello, F.J. Tackmann and J.R. Walsh, Combining Resummed Higgs Predictions Across Jet Bins, Phys. Rev. D 89 (2014) 074044 [arXiv:1312.4535] [INSPIRE].

[7] Y.L. Dokshitzer, D. Diakonov and S.I. Troian, On the Transverse Momentum Distribution of Massive Lepton Pairs, Phys. Lett. B 79 (1978) 269 [INSPIRE].

[8] G. Parisi and R. Petronzio, Small Transverse Momentum Distributions in Hard Processes, Nucl. Phys. B 154 (1979) 427 [INSPIRE].

[9] J.C. Collins, D.E. Soper and G.F. Sterman, Transverse Momentum Distribution in Drell-Yan Pair and $W$ and $Z$ Boson Production, Nucl. Phys. B 250 (1985) 199 [InSPIRE]. 
[10] C.T.H. Davies, B.R. Webber and W.J. Stirling, Drell-Yan Cross-Sections at Small Transverse Momentum, Nucl. Phys. B 256 (1985) 413 [InSPIRE].

[11] G. Altarelli, R.K. Ellis, M. Greco and G. Martinelli, Vector Boson Production at Colliders: A Theoretical Reappraisal, Nucl. Phys. B 246 (1984) 12 [InSPIRE].

[12] S. Catani, D. de Florian and M. Grazzini, Universality of nonleading logarithmic contributions in transverse momentum distributions, Nucl. Phys. B 596 (2001) 299 [hep-ph/0008184] [INSPIRE].

[13] D. de Florian and M. Grazzini, The structure of large logarithmic corrections at small transverse momentum in hadronic collisions, Nucl. Phys. B 616 (2001) 247 [hep-ph/0108273] [INSPIRE].

[14] T. Becher and M. Neubert, Drell-Yan production at small $q_{T}$, transverse parton distributions and the collinear anomaly, Eur. Phys. J. C 71 (2011) 1665 [arXiv:1007.4005] [INSPIRE].

[15] A. Idilbi, X.-d. Ji and F. Yuan, Transverse momentum distribution through soft-gluon resummation in effective field theory, Phys. Lett. B 625 (2005) 253 [hep-ph/0507196] [INSPIRE].

[16] S. Mantry and F. Petriello, Transverse Momentum Distributions from Effective Field Theory with Numerical Results, Phys. Rev. D 83 (2011) 053007 [arXiv:1007.3773] [INSPIRE].

[17] M.G. Echevarria, A. Idilbi and I. Scimemi, Factorization Theorem For Drell-Yan At Low $q_{T}$ And Transverse Momentum Distributions On-The-Light-Cone, JHEP 07 (2012) 002 [arXiv:1111.4996] [INSPIRE].

[18] H.-n. Li, Z. Li and C.-P. Yuan, QCD resummation for light-particle jets, Phys. Rev. D 87 (2013) 074025 [arXiv: 1206.1344] [INSPIRE].

[19] M. Dasgupta, K. Khelifa-Kerfa, S. Marzani and M. Spannowsky, On jet mass distributions in $Z+j e t$ and dijet processes at the LHC, JHEP 10 (2012) 126 [arXiv:1207.1640] [INSPIRE].

[20] Y.-T. Chien, R. Kelley, M.D. Schwartz and H.X. Zhu, Resummation of Jet Mass at Hadron Colliders, Phys. Rev. D 87 (2013) 014010 [arXiv:1208.0010] [InSPIRE].

[21] T.T. Jouttenus, I.W. Stewart, F.J. Tackmann and W.J. Waalewijn, Jet mass spectra in Higgs boson plus one jet at next-to-next-to-leading logarithmic order, Phys. Rev. D 88 (2013) 054031 [arXiv: 1302.0846] [INSPIRE].

[22] A. Abdesselam et al., Boosted objects: A Probe of beyond the Standard Model physics, Eur. Phys. J. C 71 (2011) 1661 [arXiv:1012.5412] [INSPIRE].

[23] A. Altheimer et al., Jet Substructure at the Tevatron and LHC: New results, new tools, new benchmarks, J. Phys. G 39 (2012) 063001 [arXiv:1201.0008] [INSPIRE].

[24] A. Altheimer et al., Boosted objects and jet substructure at the LHC. Report of BOOST2012, held at IFIC Valencia, 23rd-27th of July 2012, Eur. Phys. J. C 74 (2014) 2792 [arXiv: 1311.2708] [INSPIRE].

[25] I.W. Stewart, F.J. Tackmann and W.J. Waalewijn, N-Jettiness: An Inclusive Event Shape to Veto Jets, Phys. Rev. Lett. 105 (2010) 092002 [arXiv:1004.2489] [INSPIRE].

[26] J. Thaler and K. Van Tilburg, Identifying Boosted Objects with N-subjettiness, JHEP 03 (2011) 015 [arXiv: 1011.2268] [INSPIRE]. 
[27] J. Thaler and K. Van Tilburg, Maximizing Boosted Top Identification by Minimizing N-subjettiness, JHEP 02 (2012) 093 [arXiv: 1108.2701] [INSPIRE].

[28] A. Banfi, G.P. Salam and G. Zanderighi, Principles of general final-state resummation and automated implementation, JHEP 03 (2005) 073 [hep-ph/0407286] [INSPIRE].

[29] A.J. Larkoski, G.P. Salam and J. Thaler, Energy Correlation Functions for Jet Substructure, JHEP 06 (2013) 108 [arXiv: 1305.0007] [INSPIRE].

[30] A.J. Larkoski, I. Moult and D. Neill, Power Counting to Better Jet Observables, JHEP 12 (2014) 009 [arXiv: 1409.6298] [inSPIRE].

[31] J. Thaler and L.-T. Wang, Strategies to Identify Boosted Tops, JHEP 07 (2008) 092 [arXiv: 0806.0023] [INSPIRE].

[32] L.G. Almeida et al., Substructure of high-p $p_{T}$ Jets at the LHC, Phys. Rev. D 79 (2009) 074017 [arXiv: 0807.0234] [INSPIRE].

[33] A.J. Larkoski and J. Thaler, Unsafe but Calculable: Ratios of Angularities in Perturbative QCD, JHEP 09 (2013) 137 [arXiv:1307.1699] [INSPIRE].

[34] A.J. Larkoski, I. Moult and D. Neill, Toward Multi-Differential Cross Sections: Measuring Two Angularities on a Single Jet, JHEP 09 (2014) 046 [arXiv: 1401.4458] [INSPIRE].

[35] T. Sjöstrand, S. Mrenna and P.Z. Skands, PYTHIA 6.4 Physics and Manual, JHEP 05 (2006) 026 [hep-ph/0603175] [INSPIRE].

[36] M. Bahr et al., HERWIG++ Physics and Manual, Eur. Phys. J. C 58 (2008) 639 [arXiv: 0803.0883] [INSPIRE].

[37] A.J. Larkoski, J. Thaler and W.J. Waalewijn, Gaining (Mutual) Information about Quark/Gluon Discrimination, JHEP 11 (2014) 129 [arXiv:1408.3122] [INSPIRE].

[38] S. Frixione and B.R. Webber, Matching NLO QCD computations and parton shower simulations, JHEP 06 (2002) 029 [hep-ph/0204244] [INSPIRE].

[39] Z. Nagy and D.E. Soper, Matching parton showers to NLO computations, JHEP 10 (2005) 024 [hep-ph/0503053] [INSPIRE].

[40] S. Frixione, P. Nason and C. Oleari, Matching NLO QCD computations with Parton Shower simulations: the POWHEG method, JHEP 11 (2007) 070 [arXiv:0709.2092] [INSPIRE].

[41] S. Alioli, P. Nason, C. Oleari and E. Re, A general framework for implementing NLO calculations in shower Monte Carlo programs: the POWHEG BOX, JHEP 06 (2010) 043 [arXiv:1002.2581] [INSPIRE].

[42] S. Alioli et al., Combining Higher-Order Resummation with Multiple NLO Calculations and Parton Showers in GENEVA, JHEP 09 (2013) 120 [arXiv:1211.7049] [INSPIRE].

[43] K. Hamilton, P. Nason, E. Re and G. Zanderighi, NNLOPS simulation of Higgs boson production, JHEP 10 (2013) 222 [arXiv:1309.0017] [INSPIRE].

[44] S. Alioli et al., Matching Fully Differential NNLO Calculations and Parton Showers, JHEP 06 (2014) 089 [arXiv:1311.0286] [INSPIRE].

[45] S. Hoeche, Y. Li and S. Prestel, Drell-Yan lepton pair production at NNLO QCD with parton showers, arXiv:1405.3607 [INSPIRE].

[46] I.W. Stewart, F.J. Tackmann and W.J. Waalewijn, Factorization at the LHC: From PDFs to Initial State Jets, Phys. Rev. D 81 (2010) 094035 [arXiv:0910.0467] [INSPIRE]. 
[47] F.J. Tackmann, J.R. Walsh and S. Zuberi, Resummation Properties of Jet Vetoes at the LHC, Phys. Rev. D 86 (2012) 053011 [arXiv: 1206.4312] [InSPIRE].

[48] C.W. Bauer, S. Fleming and M.E. Luke, Summing Sudakov logarithms in $B \rightarrow X_{s} \gamma$ in effective field theory, Phys. Rev. D 63 (2000) 014006 [hep-ph/0005275] [inSPIRE].

[49] C.W. Bauer, S. Fleming, D. Pirjol and I.W. Stewart, An Effective field theory for collinear and soft gluons: Heavy to light decays, Phys. Rev. D 63 (2001) 114020 [hep-ph/0011336] [INSPIRE].

[50] C.W. Bauer and I.W. Stewart, Invariant operators in collinear effective theory, Phys. Lett. B 516 (2001) 134 [hep-ph/0107001] [INSPIRE].

[51] C.W. Bauer, D. Pirjol and I.W. Stewart, Soft collinear factorization in effective field theory, Phys. Rev. D 65 (2002) 054022 [hep-ph/0109045] [INSPIRE].

[52] C.W. Bauer, F.J. Tackmann, J.R. Walsh and S. Zuberi, Factorization and Resummation for Dijet Invariant Mass Spectra, Phys. Rev. D 85 (2012) 074006 [arXiv:1106.6047] [InSPIRE].

[53] A. Jain, M. Procura and W.J. Waalewijn, Fully-Unintegrated Parton Distribution and Fragmentation Functions at Perturbative $k_{T}$, JHEP 04 (2012) 132 [arXiv:1110.0839] [INSPIRE].

[54] J.C. Collins, T.C. Rogers and A.M. Stasto, Fully unintegrated parton correlation functions and factorization in lowest-order hard scattering, Phys. Rev. D 77 (2008) 085009 [arXiv: 0708.2833] [INSPIRE].

[55] T.C. Rogers, Next-to-Leading Order Hard Scattering Using Fully Unintegrated Parton Distribution Functions, Phys. Rev. D 78 (2008) 074018 [arXiv:0807.2430] [InSPIRE].

[56] S. Mantry and F. Petriello, Factorization and Resummation of Higgs Boson Differential Distributions in Soft-Collinear Effective Theory, Phys. Rev. D 81 (2010) 093007 [arXiv:0911.4135] [INSPIRE].

[57] ATLAS collaboration, M. Myska, Measurement of the double parton scattering in $W+2$ jets production at $\sqrt{s}=7 \mathrm{TeV}$ with the ATLAS detector, EPJ Web Conf. 60 (2013) 20013 [INSPIRE].

[58] CMS collaboration, Study of double parton scattering using $W+2$-jet events in proton-proton collisions at $\sqrt{s}=7 \mathrm{TeV}, \mathrm{JHEP} 03$ (2014) 032 [arXiv:1312.5729] [INSPIRE].

[59] M. Cacciari, G.P. Salam and S. Sapeta, On the characterisation of the underlying event, JHEP 04 (2010) 065 [arXiv:0912.4926] [INSPIRE].

[60] M. Diehl, D. Ostermeier and A. Schafer, Elements of a theory for multiparton interactions in $Q C D$, JHEP 03 (2012) 089 [arXiv:1111.0910] [INSPIRE].

[61] M.G. Ryskin and A.M. Snigirev, A Fresh look at double parton scattering, Phys. Rev. D 83 (2011) 114047 [arXiv:1103.3495] [INSPIRE].

[62] A.V. Manohar and W.J. Waalewijn, What is Double Parton Scattering?, Phys. Lett. B 713 (2012) 196 [arXiv: 1202.5034] [INSPIRE].

[63] J.R. Gaunt, Single Perturbative Splitting Diagrams in Double Parton Scattering, JHEP 01 (2013) 042 [arXiv: 1207.0480] [INSPIRE].

[64] B. Blok, Y. Dokshitzer, L. Frankfurt and M. Strikman, Perturbative QCD correlations in multi-parton collisions, Eur. Phys. J. C 74 (2014) 2926 [arXiv:1306.3763] [INSPIRE]. 
[65] S.D. Ellis, C.K. Vermilion, J.R. Walsh, A. Hornig and C. Lee, Jet Shapes and Jet Algorithms in SCET, JHEP 11 (2010) 101 [arXiv:1001.0014] [INSPIRE].

[66] T.T. Jouttenus, I.W. Stewart, F.J. Tackmann and W.J. Waalewijn, The Soft Function for Exclusive N-Jet Production at Hadron Colliders, Phys. Rev. D 83 (2011) 114030 [arXiv:1102.4344] [INSPIRE].

[67] M. Dasgupta and G.P. Salam, Resummation of nonglobal QCD observables, Phys. Lett. B 512 (2001) 323 [hep-ph/0104277] [inSPIRE].

[68] M. Dasgupta and G.P. Salam, Resummed event shape variables in DIS, JHEP 08 (2002) 032 [hep-ph/0208073] [INSPIRE].

[69] I.W. Stewart, Lectures on the Soft-Collinear Effective Theory, MIT Open Course Ware, Effective Field Theory (Spring 2013), http://ocw.mit.edu/courses/physics/8-851-effectivefield-theory-spring-2013/lecture-notes/MIT8_851S13_scetnotes.pdf.

[70] T. Becher, A. Broggio and A. Ferroglia, Introduction to Soft-Collinear Effective Theory, arXiv:1410.1892 [INSPIRE].

[71] J.-y. Chiu, A. Jain, D. Neill and I.Z. Rothstein, The Rapidity Renormalization Group, Phys. Rev. Lett. 108 (2012) 151601 [arXiv:1104.0881] [INSPIRE].

[72] J.-Y. Chiu, A. Jain, D. Neill and I.Z. Rothstein, A Formalism for the Systematic Treatment of Rapidity Logarithms in Quantum Field Theory, JHEP 05 (2012) 084 [arXiv:1202.0814] [INSPIRE].

[73] J.-y. Chiu, F. Golf, R. Kelley and A.V. Manohar, Electroweak Corrections in High Energy Processes using Effective Field Theory, Phys. Rev. D 77 (2008) 053004 [arXiv:0712.0396] [INSPIRE].

[74] A.V. Manohar and I.W. Stewart, The zero-bin and mode factorization in quantum field theory, Phys. Rev. D 76 (2007) 074002 [hep-ph/0605001] [INSPIRE].

[75] G.T. Bodwin, S.J. Brodsky and G.P. Lepage, Initial State Interactions and the Drell-Yan Process, Phys. Rev. Lett. 47 (1981) 1799 [INSPIRE].

[76] J.C. Collins, D.E. Soper and G.F. Sterman, Soft Gluons and Factorization, Nucl. Phys. B 308 (1988) 833 [inSPIRE].

[77] J.R. Gaunt, Glauber Gluons and Multiple Parton Interactions, JHEP 07 (2014) 110 [arXiv:1405.2080] [INSPIRE].

[78] E. Laenen, G.F. Sterman and W. Vogelsang, Recoil and threshold corrections in short distance cross-sections, Phys. Rev. D 63 (2001) 114018 [hep-ph/0010080] [InSPIRE].

[79] I.W. Stewart, F.J. Tackmann and W.J. Waalewijn, The Quark Beam Function at NNLL, JHEP 09 (2010) 005 [arXiv: 1002.2213] [INSPIRE].

[80] R. Kelley, M.D. Schwartz, R.M. Schabinger and H.X. Zhu, The two-loop hemisphere soft function, Phys. Rev. D 84 (2011) 045022 [arXiv:1105.3676] [InSPIRE].

[81] A. Hornig, C. Lee, I.W. Stewart, J.R. Walsh and S. Zuberi, Non-global Structure of the $O\left(\alpha_{s}^{2}\right)$ Dijet Soft Function, JHEP 08 (2011) 054 [arXiv: 1105.4628] [INSPIRE].

[82] A. Hornig, C. Lee, J.R. Walsh and S. Zuberi, Double Non-Global Logarithms In-N-Out of Jets, JHEP 01 (2012) 149 [arXiv:1110.0004] [INSPIRE].

[83] A.V. Manohar, Deep inelastic scattering as $x \rightarrow 1$ using soft collinear effective theory, Phys. Rev. D 68 (2003) 114019 [hep-ph/0309176] [INSPIRE]. 
[84] C.W. Bauer, C. Lee, A.V. Manohar and M.B. Wise, Enhanced nonperturbative effects in $Z$ decays to hadrons, Phys. Rev. D 70 (2004) 034014 [hep-ph/0309278] [INSPIRE].

[85] J.R. Gaunt and M. Stahlhofen, The Fully-Differential Quark Beam Function at NNLO, JHEP 12 (2014) 146 [arXiv:1409.8281] [INSPIRE].

[86] J.C. Collins and D.E. Soper, Parton Distribution and Decay Functions, Nucl. Phys. B 194 (1982) 445 [INSPIRE].

[87] M. Ritzmann and W.J. Waalewijn, Fragmentation in Jets at NNLO, Phys. Rev. D 90 (2014) 054029 [arXiv:1407.3272] [INSPIRE].

[88] J. Collins, Cambridge Monographs on Particle Physics, Nuclear Physics and Cosmology. Book 32: Foundations of perturbative QCD, Cambridge University Press, Cambridge U.K. (2011).

[89] M.D. Schwartz, Resummation and NLO matching of event shapes with effective field theory, Phys. Rev. D 77 (2008) 014026 [arXiv:0709.2709] [InSPIRE].

[90] S. Fleming, A.H. Hoang, S. Mantry and I.W. Stewart, Top Jets in the Peak Region: Factorization Analysis with NLL Resummation, Phys. Rev. D 77 (2008) 114003 [arXiv:0711.2079] [INSPIRE].

[91] G. Parisi, Summing Large Perturbative Corrections in QCD, Phys. Lett. B 90 (1980) 295 [INSPIRE].

[92] G.F. Sterman, Summation of Large Corrections to Short Distance Hadronic Cross-Sections, Nucl. Phys. B 281 (1987) 310 [inSPIRE].

[93] L. Magnea and G.F. Sterman, Analytic continuation of the Sudakov form-factor in QCD, Phys. Rev. D 42 (1990) 4222 [inSPIRE].

[94] T.O. Eynck, E. Laenen and L. Magnea, Exponentiation of the Drell-Yan cross-section near partonic threshold in the DIS and MS-bar schemes, JHEP 06 (2003) 057 [hep-ph/0305179] [INSPIRE].

[95] C. Balzereit, T. Mannel and W. Kilian, Evolution of the light cone distribution function for a heavy quark, Phys. Rev. D 58 (1998) 114029 [hep-ph/9805297] [INSPIRE].

[96] M. Neubert, Renormalization-group improved calculation of the $B \rightarrow X_{s} \gamma$ branching ratio, Eur. Phys. J. C 40 (2005) 165 [hep-ph/0408179] [InSPIRE].

[97] Z. Ligeti, I.W. Stewart and F.J. Tackmann, Treating the $b$ quark distribution function with reliable uncertainties, Phys. Rev. D 78 (2008) 114014 [arXiv:0807.1926] [INSPIRE].

[98] A.V. Manohar and W.J. Waalewijn, A QCD Analysis of Double Parton Scattering: Color Correlations, Interference Effects and Evolution, Phys. Rev. D 85 (2012) 114009 [arXiv: 1202.3794] [INSPIRE].

[99] L.G. Almeida et al., Comparing and counting logs in direct and effective methods of $Q C D$ resummation, JHEP 04 (2014) 174 [arXiv:1401.4460] [INSPIRE].

[100] R. Abbate, M. Fickinger, A.H. Hoang, V. Mateu and I.W. Stewart, Thrust at $N^{3} L L$ with Power Corrections and a Precision Global Fit for $\alpha_{s}\left(m_{Z}\right)$, Phys. Rev. D 83 (2011) 074021 [arXiv:1006.3080] [INSPIRE].

[101] M. Procura, W.J. Waalewijn and L. Zeune, in preparation.

[102] C.F. Berger, T. Kucs and G.F. Sterman, Event shape/energy flow correlations, Phys. Rev. D 68 (2003) 014012 [hep-ph/0303051] [INSPIRE]. 
[103] S. Catani, G. Turnock and B.R. Webber, Jet broadening measures in $e^{+} e^{-}$annihilation, Phys. Lett. B 295 (1992) 269 [inSPIRE].

[104] Y.L. Dokshitzer, A. Lucenti, G. Marchesini and G.P. Salam, On the QCD analysis of jet broadening, JHEP 01 (1998) 011 [hep-ph/9801324] [INSPIRE].

[105] A.J. Larkoski, D. Neill and J. Thaler, Jet Shapes with the Broadening Axis, JHEP 04 (2014) 017 [arXiv: 1401.2158] [INSPIRE].

[106] D. Bertolini, T. Chan and J. Thaler, Jet Observables Without Jet Algorithms, JHEP 04 (2014) 013 [arXiv: 1310.7584] [INSPIRE].

[107] O.V. Tarasov, A.A. Vladimirov and A.Y. Zharkov, The Gell-Mann-Low Function of QCD in the Three Loop Approximation, Phys. Lett. B 93 (1980) 429 [INSPIRE].

[108] S.A. Larin and J.A.M. Vermaseren, The Three loop QCD $\beta$-function and anomalous dimensions, Phys. Lett. B 303 (1993) 334 [hep-ph/9302208] [INSPIRE].

[109] G.P. Korchemsky and A.V. Radyushkin, Renormalization of the Wilson Loops Beyond the Leading Order, Nucl. Phys. B 283 (1987) 342 [InSPIRE].

[110] S. Moch, J.A.M. Vermaseren and A. Vogt, The Three loop splitting functions in QCD: The Nonsinglet case, Nucl. Phys. B 688 (2004) 101 [hep-ph/0403192] [INSPIRE].

[111] S. Moch, J.A.M. Vermaseren and A. Vogt, The Quark form-factor at higher orders, JHEP 08 (2005) 049 [hep-ph/0507039] [INSPIRE].

[112] S. Moch, J.A.M. Vermaseren and A. Vogt, Three-loop results for quark and gluon form-factors, Phys. Lett. B 625 (2005) 245 [hep-ph/0508055] [INSPIRE].

[113] A. Vogt, S. Moch and J.A.M. Vermaseren, The Three-loop splitting functions in QCD: The Singlet case, Nucl. Phys. B 691 (2004) 129 [hep-ph/0404111] [InSPIRE].

[114] A. Idilbi, X.-d. Ji, J.-P. Ma and F. Yuan, Threshold resummation for Higgs production in effective field theory, Phys. Rev. D 73 (2006) 077501 [hep-ph/0509294] [INSPIRE].

[115] A. Idilbi, X.-d. Ji and F. Yuan, Resummation of threshold logarithms in effective field theory for DIS, Drell-Yan and Higgs production, Nucl. Phys. B 753 (2006) 42 [hep-ph/0605068] [INSPIRE].

[116] T. Becher, M. Neubert and B.D. Pecjak, Factorization and Momentum-Space Resummation in Deep-Inelastic Scattering, JHEP 01 (2007) 076 [hep-ph/0607228] [INSPIRE].

[117] T. Becher and M.D. Schwartz, Direct photon production with effective field theory, JHEP 02 (2010) 040 [arXiv:0911.0681] [INSPIRE].

[118] T. Gehrmann, T. Luebbert and L.L. Yang, Calculation of the transverse parton distribution functions at next-to-next-to-leading order, JHEP 06 (2014) 155 [arXiv:1403.6451] [INSPIRE]. 